\title{
DIGITALCOMMONS
}

@WAYNESTATE-

Wayne State University

Wayne State University Dissertations

January 2014

\section{An Analysis Of Self: The Development And Assessment Of A Measure Of Selfobject Needs}

Eamonn Arble

Wayne State University, Eurytus@hotmail.com

Follow this and additional works at: https://digitalcommons.wayne.edu/oa_dissertations

Part of the Clinical Psychology Commons

\section{Recommended Citation}

Arble, Eamonn, "An Analysis Of Self: The Development And Assessment Of A Measure Of Selfobject Needs" (2014). Wayne State University Dissertations. 1005.

https://digitalcommons.wayne.edu/oa_dissertations/1005

This Open Access Dissertation is brought to you for free and open access by DigitalCommons@WayneState. It has been accepted for inclusion in Wayne State University Dissertations by an authorized administrator of DigitalCommons@WayneState. 


\section{AN ANALYSIS OF SELF: \\ THE DEVELOPMENT AND ASSESSMENT OF A MEASURE OF SELFOBJECT NEEDS}

by

EAMONN ARBLE

DISSERTATION

Submitted to the Graduate School

of Wayne State University,

Detroit, Michigan

in partial fulfillment of the requirements

for the degree of

DOCTOR OF PHILOSOPHY

2014

MAJOR: PSYCHOLOGY

Approved by:

Advisor

Date 


\section{TABLE OF CONTENTS}

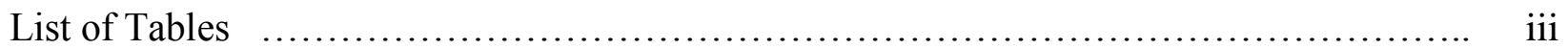

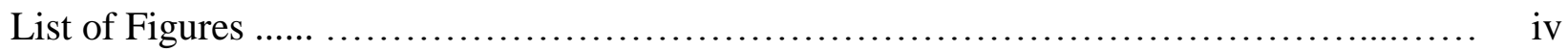

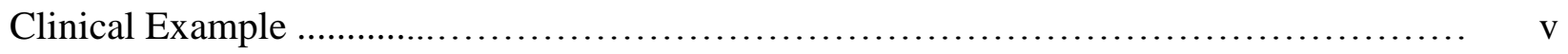

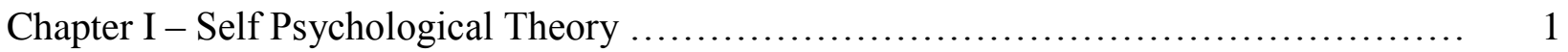

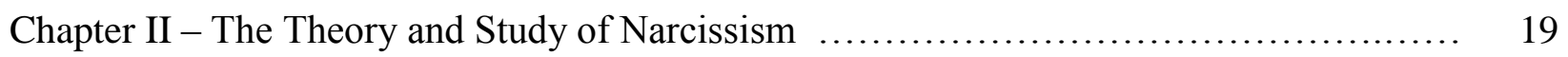

Chapter III - Self Psychology as Not Entirely Esoteric ............................... 38

Chapter IV - Attachment and Self Psychology .......................................... 43

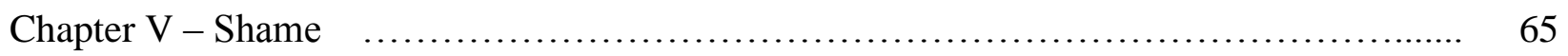

Chapter VI - Existing Self Psychology Measures ....................................... 72

Chapter VII - Problem Statement ................................................ 80

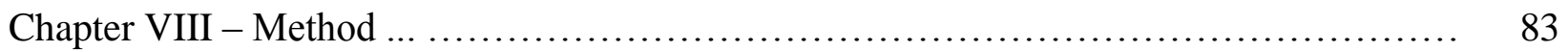

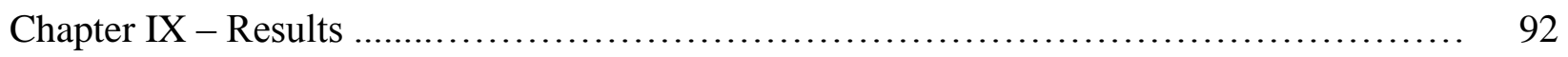

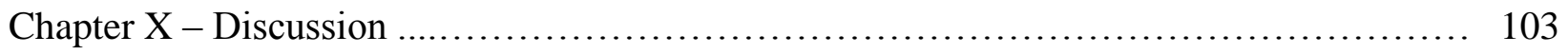

Chapter XI - Making Use of the Results ............................................ 125

Appendix A - Initial AESOP Scale Items....................................................................... 153

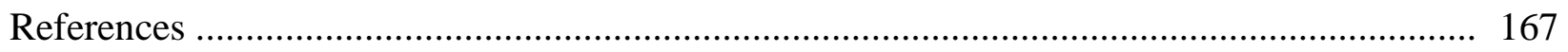

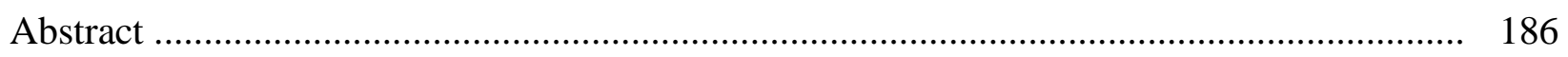

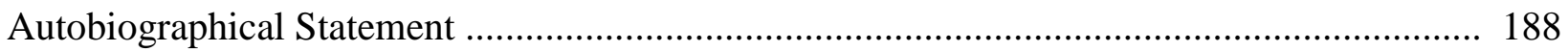




\section{LIST OF TABLES}

Table 1. Potential items for Idealizing subscale................................. 128

Table 2. Potential Items for Mirror subscale .................................................................. 131

Table 3. Potential items for Twinship subscale. ....................................................... 133

Table 4. Rotated factor solution of remaining AESOP items. ........................................ 135

Table 5. Reliability statistics by selfobject need. ........................................................ 137

Table 6. Confirmatory factor analysis measurement loadings. ....................................... 139

Table 7. Summary of discriminant analysis. ......................................................... 140

Table 8. Summary of discriminant analysis: differential effects by sub-scale. ................... 141

Table 9. Rotated factor loadings (pattern matrix) of SONI items. .................................... 142

Table 10. Correlations amongst all assessed measures. ............................................. 144 


\section{LIST OF FIGURES}

Figure 1: Comparison of AESOP clusters on multiple outcomes. 


\section{CLINICAL EXAMPLE}

Adapted from McLean's (2007) submission for the "Psychotherapy Rounds" series.

Mr. Taylor was a 35-year-old twice-divorced man who was the father of four children. Mr. Taylor entered treatment citing difficulties in his interpersonal relationships. Specifically, he was confused as to why he seemed unable to maintain any social relationships. When asked to detail the emotional consequence of his lack of friendships, Mr. Taylor stated that there were many. He described himself as "often depressed," citing low energy and motivation, an inability to concentrate on tasks, a lack of interest in his hobbies, disrupted sleep, and a sense of shame and embarrassment. When asked to clarify the latter, Mr. Taylor related a sense of frustration about his "constant failure" to connect with people. However, Mr. Taylor was ill soon to begin a new job, and expressed a sense of optimism that the new job would allow him to "start again." Mr. Taylor was born to working class parents, though his parents divorced before he was born. His father left the state while Mr. Taylor's mother was still pregnant with him, and his father never attempted to contact Mr. Taylor or Mr. Taylor's mother again. Mr. Taylor's mother was forced to work two jobs in order to support herself and Mr. Taylor, and she further leaned upon family members to assist her in taking care of him. As a result, Mr. Taylor would live with various local family members for weeks at a time, essentially moving across a series of transitory residences.

At the age of 18, Mr. Taylor married his high school sweetheart and the relationship lasted for four years and produced two children. The two became divorced at Mr. Taylor's insistence. Mr. Taylor recalled becoming "bored" towards the end of the relationship, stating that he "felt like there was something missing in her, it was like she was unimpressive in some way." Mr. Taylor would marry his second wife three years later, a marriage that would last for 
nine years. "It was a good marriage, but I felt like she didn't appreciate me. She didn't really understand me and she only seemed to focus on her own needs." This relationship would result in another two children.

The initial weeks of therapy were dedicated to assessing and improving Mr. Taylor's social skills, as well as confronting the emotional consequences of his perceived difficulties, utilizing a cognitive-behavioral approach. Mr. Taylor's evaluations of past interactions were reviewed, with particular emphasis placed on his negative interpretations of mundane events. For example, when Mr. Taylor concluded that his coworkers carried a strong resentment of him because they did not invite him to various activities, he was encouraged to explore the possibility that their actions might be better understood as a reflection of the fact that they did not know him well. Mr. Taylor's attempted solutions to his social isolation were also explored, and it was concluded by both Mr. Taylor and the therapist, that in response to the perceived slights of his coworkers, Mr. Taylor became sullen and withdrawn, which in turn decreased the likelihood of establishing friendships. More proactive strategies to increase engagement with others were discussed in session and were reviewed with lengthy role-playing sessions and homework assignments.

Yet soon after beginning the new job, Mr. Taylor began to feel "left out" at work. "I don't understand why they don't seem to like me. I mean, I have more going for me than almost all of them put together." Mr. Taylor further emphasized the "humiliating" experience of being “exposed as a little fish." "All of my recent performance reviews have been 'average' or 'fine.' I feel like I've been knocked off a cliff, you know, it makes me really question myself."

As the therapy progressed, Mr. Taylor became increasingly concerned over his social skills, growing frustrated that he could rehearse skills in session, but his connections with other 
people didnot appear to be improving. Congruently, the therapist began to develop a sense of Mr. Taylor's core beliefs, which the therapist felt were largely organized around themes of being "unworthy of other's people's love". However, the therapist found it difficult to progress to these core beliefs in session, as the therapeutic alliance appeared tentative at best, and Mr. Taylor had begun to ask the therapist for an assessment of his social skills every session, directly asking: "How do YOU see me?" Although Mr. Taylor invited such commentary, he would become highly distressed at even a hint of criticism from the therapist. This distress would often turn to anger, and Mr. Taylor frequently responded by questioning the therapist's credibility and skill.

Mr. Taylor also expressed a sense of exasperation at the therapist's unwillingness to share personal information. "You know, just because you're the therapist doesn't mean you're perfect. We're both human beings here, and when I ask about you and your life, it's just because I want to know you. I don't understand why you feel like we can't connect in that way." Despite these objections, Mr. Taylor attended sessions regularly, and would at times appear highly engaged in the treatment.

Concurrent to his weekly sessions, the therapist had also referred Mr. Taylor to a social skills group offered at a nearby clinic. Mr. Taylor attended the group despite his frequently expressed concern regarding its effectiveness. Mr. Taylor reported that the other group members did not appear to enjoy his company, and that the group leader "doesn't seem to know what the heck is going on." Despite these objections, Mr. Taylor attended the group for 10 sessions before deciding to leave. Several sessions after terminating from group, Mr. Taylor abruptly expressed a sense of anger at the therapist. "Those people were a messed up bunch of losers and you sent me there. I almost left on the first day, but I decided to stick it out. But it was like you didn't care about what I did or what I went through. We only talked about the skills part of it, 
but you never even acknowledged the fact that I hung in with those crazy people for over two months."

\section{Case Analysis}

The case of Mr. Taylor is a difficult clinical challenge. In some ways, he displays strong insight into his difficulties, as he operates with a sense of his struggles to interact in a meaningful way with others. He is also able to connect with painful affect in session, detailing his emotional reactions to things such as his performance feedback. He further demonstrates an ability to work at developing particular skills, as well as a willingness to challenge himself by attending a therapy group that he dislikes.

Yet there is something about Mr. Taylor that seems to be incomplete. His need for closeness is thwarted by his style of interacting with others, much in the same fashion his sense of immodesty is contrasted against his severe self-criticisms. Further, the sense of hurt and anger he displays toward the therapist can be somewhat surprising, as the therapist does not appear to be offering any notable provocations. It is almost as though Mr. Taylor is responding to a perceived shortcoming on the part of the therapist, as if the therapist has failed to offer some needed support.

And indeed, the notion of lacking such needed support represents the focus of the proposed investigation. Mr. Taylor's treatment and history is offered as an example of a type of client issues that are the central target of a specific therapeutic approach, namely, that of self psychology. Self psychology may perhaps best be defined as a theory of need. According to the theory first advanced by Heinz Kohut $(1971,1977,1984)$, mental health is the result of a series of positive interactions with one's caregivers and meaningful individuals throughout development. These interactions allow the child to confidently and openly connect with others, 
thereby establishing a reciprocal relationship between the experience of one's sense of self and positive emotional experiences. In other words, interacting with others in an emotionally fulfilling way gives the child a sense of strength and purpose, which in turn grants the child the confidence to seek out similar interactions in the future. In Kohut's understanding, one's sense of self necessarily arises in the context of meaningful relationships, and it is through these meaningful relationships our innate psychological needs can be met.

Conversely, those who do not have such needs met develop a sense of self that is not filled with strength or value; instead, it is characterized by a sense of weakness and vulnerability. This resulting vulnerability affects the child (and later, the adult's) ability to connect with others, and a dysfunctional style of relating with others grows as a means to defend against one's own insecurity. The reliance on such a defensive style makes it increasingly difficult for the individual to have these needs met, thus leaving them at risk for feelings of depression, rage, low self-esteem, and many other psychological difficulties.

The identification and explanation of these needs would thus become the central aspect of self psychology's clinical conceptualization model and treatment focus. The proposition that unmet needs is the core of psychopathology stands in contrast with the drive theory of traditional Freudian analysis, the notion of maladaptive patterns of thought in cognitive therapy, as well as the importance of reinforcement principles emphasized in behavioral models. In essence, self psychology stands as a unique theory of clinical psychology and treatment.

Yet despite its somewhat recent development, Kohut's theory of self psychology has proven quite influential. The fields of clinical psychology (Hartman, 2009),psychiatry(e.g., Gabbard, 2000), and social work (Chenot, 1998) have all witnessed the use of self psychological techniques. It has been employed in both therapy (e.g., Lee \& Martin, 1991) and psychological 
assessment (e.g., Silverstein, 1999), and has been additionally applied with children (Suth, 1996), the elderly (Muslin, 1993), families (Leone, 2001), and within group therapy (Stone, 1992). The clinical problems it has been utilized in the treatment of are similarly broad, and include borderline personality disorder (Palombo, 1983), mourning (Paradis, 1993), psychotic disorders (Garfield, 2001), eating disorders (Barth, 1988), trauma (Josephs, 1992), substance abuse (Ulman \& Paul, 1989), sexual dysfunction (Giugliano, 2011), and most notably, narcissistic personality disorder (Silverstein, 2007).

However, despite its interesting theoretical foundations, its perceived clinical utility, and its use in a wide variety of contexts, the self psychology proposed by Kohut has been hampered by three primary difficulties. First, Kohut's writings are both theoretically complex and difficult to understand, a concession even made by proponents of the theory (e.g., Siegel, 1996). Second, with the exception of Kohut's conceptualization of the narcissistic personality disorder, there has been scant research on the myriad of constructs proposed by the self psychology theory. Finally, Kohut's reliance on the importance of early childhood experiences shares much in common with other developmental perspectives, most notably, attachment theory. Thus, even if the validity of self psychology's proposals were to be established, its incremental validity would remain in question.

Self psychology as a theory is both broad and multifaceted, and thus, a comprehensive review of the theory and its multitude of interpretations is beyond the scope of this proposal. Instead, I will turn our attention to the needs at the core of the theory: selfobject needs. In the text that follows, I will present an overview of self psychological theory, the available research involving its concepts and constructs, as well as its relationship with the apparently congruent 
theory of attachment. Finally, an empirical method aimed at developing and testing a new measure of selfobject needs will be proposed.

\section{Organization of the Introduction}

The introductory material is organized into six chapters, each with its own subheadings.

Chapter I (p. 9) provides an introductory discussion on the theory of self psychology. As has been mentioned, self psychology's original texts are both dense and confusing, and this chapter is dedicated to providing a thorough and relatable explanation of the theory. Although the discussion is far from comprehensive, the number of unique concepts presented can make the material somewhat difficult. To that end, the material detailed under the Selfobject Needs heading is the single most important aspect, with particular focus merited by the Mirroring, Idealizing, and Twinship needs that will be assessed by the study's proposed measure.

Chapter II (p. 19) examines the research related to the proposed "covert" form of narcissism. The study of narcissism is crucial for two reasons. First, Heinz Kohut's self psychology was initially developed as a tool for the treatment of narcissistic personalities, before being expanded to the more broadly applied theory of contemporary usage. The utility of this expansion remains unproven. Second, a notable shortcoming of self psychology is its lack of corresponding research. However, Kohut's views on narcissism are an important exception to this failing, and provide the only rigorously researched aspect of self psychological theory.

Chapter III (p. 38) is a brief consideration of the fact that, although self psychology's concepts may appear byzantine at first, the central premises articulated by Kohut actually share numerous similarities with other contemporary therapeutic models.

Chapter IV (p. 43) briefly reviews the theory of attachment. Attachment theory and self psychology are often viewed as congruent, though one might also contend that the two are 
actually redundant. The overlapping relations of attachment theory and self psychology is articulated to clarify hypotheses regarding convergent and discriminant validity between the two theories.

Chapter V (p. 65) offers a discussion of the concept of shame. Shame is a critical component of self psychological theory, and will be an outcome of great interest in the present study. Shame's relationship with self psychology, attachment, and narcissism is considered.

Chapter VI (p. 72) describes the existing self psychology measures and their relevant research. One particular measure, the SONI, is given the greatest amount of attention.

At the end of each chapter is a brief italicized paragraph summarizing the chapter's main points and explaining its relevance for the present study. Examining these summary paragraphs can be a useful way to quickly review the present material. 


\section{CHAPTER I}

\section{SELF PSYCHOLOGICAL THEORY}

\section{The Development of Self Psychology}

In many respects, the self psychology advanced by Heinz Kohut in The Analysis of the Self (1971), reflected an expansion of the psychoanalytic schools of ego and drive theory. When he wrote it, Kohut was a training analyst at the Chicago Psychoanalytic Institute, working from the traditional Freudian approach. However, in the pivotal case of "Miss F.", Kohut (1971) detailed his increasing frustration with the therapeutic modalities of the time.

Miss F., a young woman in her twenties, came to treatment citing broad and unspecific concerns. Miss F. superficially appeared well-adjusted, as she stated that she had progressed in her career and was able to detail several romantic relationships that she had cultivated in her adult life. However, Miss F. seemed unnerved by a sense of uncertainty regarding her own thoughts and feelings, and she expressed a feeling of being fundamentally different and separate from those around her. In times when this uncertainty became prominent in her mind, Miss $\mathrm{F}$. demonstrated notable and sudden shifts in mood. Further, Kohut observed, she appeared to lean on her interpersonal relationships as a source of escape from these worries; indeed, an individual's ability to comfort her in this regard appeared to be Miss F's primary motivation for forging a relationship with them.

Speaking to the course of his work with her, Kohut observed a curious pattern in their relationship. Miss F. frequently began her sessions in a generally positive mood, speaking freely about the various difficulties and events transpiring in her life. Kohut would typically respond with silence, allowing Miss F. to explore her associations without interference. However, Miss F. reacted to this silence with bouts of anger and disappointment, adamantly faulting Kohut for 
his failure to offer her emotional support.

Though Miss F.'s angry outbursts were initially surprising for Kohut, he began to anticipate them as a regular occurrence during their sessions. Kohut responded to these emotional upheavals by describing them to Miss F. in terms of traditional transference formulations, wherein the emotions she experienced towards him were explained as actually reflecting some of the emotions she had for other significant persons in her life. Yet such formulations neither resonated with Miss F., nor alleviated her distress. Despite his systematic application of traditional transference analyses, it did not appear as though Kohut, acting as Miss F.'s therapist, was experiencing the emotional content that was truly meant for another.

Recognizing the fruitlessness of his efforts, Kohut attempted a number of solutions. With some experimentation Kohut discovered that by offering an empathetic response to Miss F.'s concerns, she would become notably relaxed and comforted. However, if he attempted to build interpretations upon his empathic reflections, Miss F would return to her angry stance, and the comfort derived from Kohut's empathy would dissolve. Further, Kohut soon found that Miss F. required a specific form of empathic response, and his deviation from this response was perceived as threatening and hostile by Miss F.

After some reflection, Kohut theorized that the voice expressed by Miss F. (both in terms of its need and its tone), reflected an unspoken voice from her childhood. Kohut speculated that the voice itself represented a previously unspoken need, and that the patient required, not only to speak it, but to have the therapist hear it and respond to it in a particular way. Specifically, Kohut posited that Miss F. was enacting a particular childhood demand, a frustrated desire to be heard, recognized, and appreciated. What Miss F. desired, Kohut believed, was to display her abilities and sense of worth, and to have Kohut demonstrate approval of her by echoing her 
words and empathically responding to them. Kohut described this process of hearing the patient's "need for admiration," and empathically reflecting it back to the patient, as the “mirroring transference," a core concept of Kohut's (1971) "selfpsychology" as reflected in the following quote:

“After a prolonged period of ignorance and misunderstanding during which I was inclined to argue with the patient about the correctness of my interpretations and to suspect the presence of stubborn, hidden resistances, I came to the crucial recognition that the patient demanded a specific response....and...completely rejected any other (p. 285)."

The notion of the patient placing specific demands on the analyst, such as Miss F.'s need to be heard, supported, and admired, was also becoming evident in Kohut's analysis of his counter transference reactions. Kohut's reactions to Miss F. had ranged from exasperation to confusion, but perhaps his most consistent counter transference reaction was that of boredom. During sessions with Miss F., Kohut struggled to stay focused, and often found his mind drifting to other topics and patients. In no small part, Kohut felt that in their sessions Miss F. was speaking to him, not as a unique individual, but almost as a tool to be used in a specific way (i.e., to provide the mirroring she required).

This notion of the therapist serving such a psychological function for the patient fascinated Kohut, and he began to examine his sessions with other patients for the same process. Repeatedly, Kohut noticed that his patients were looking to him to provide the psychic functions that they were incapable of providing for themselves. Throughout their sessions, the patients would seek to act in a matter of capable of eliciting the needed response from the therapist. In so doing, the patient could lean on the therapist to bolster an incomplete sense of self. 
Kohut began to conceptualize this use of another person (in this instance, the therapist) as a means to shore up one's own sense of self as something he referred to as a selfobject (initially written as "self-object"). Kohut described the selfobject as an object (most typically, another person) that is perceived as part of one's own sense of self, as opposed to an independent entity. Kohut posited that we are born with an innate need for connectedness with others (i.e., objects), and through these connections one's sense of self is developed. Throughout one's life, Kohut speculated, selfobjects are called upon to perform a restorative function, namely, as a means of bolstering one's sense of self and helping to regulate one's self-esteem. This experience of being used as a selfobject, as Miss F. had required of him, was a disconcerting feeling for Kohut, but one he began to see as increasingly important. Given that selfobjects were posited as a critical component of development, and that Kohut had clinically observed the clinical utility of serving as a selfobject, it appeared that the concept of unmet selfobject needs (such as the need for mirroring) would serve as a core component of the psychopathology Kohut observed.

\section{Selfobject Definition}

The notion of a selfobject is probably the single most critical concept in the theory of self psychology. Kohut posited that the self serves as the core of personality and motivation (Kohut, 1977). The self is not a static trait. It grows out of each person's unique, innate, developingpsychological needs and their experiences of satisfaction and frustration (Lessem, 2005). It is through this process that a core sense of self develops. These ideas were a marked deviation from Freud's (1927) position that personality and behavior are ultimately explained by aggressive and sexual drives.

Kohut initially described selfobjects as each individual's perceived interactions with others that are experienced as part of the self, as opposed to a separate entity. Kohut would later 
alter the concept of the selfobject to focus, not on the outside object or person, but rather, on the function that object or person played in maintaining one's sense of self. As described by Wolf (1988), the selfobject represents an intrapsychic experience, as opposed to an interpersonal relationship between the self and external objects. In other words, the selfobject refers to the function the object performs for the self, as opposed to the object itself. The self requires the sustenance provided by positive interactions with others (most notably the child's caregivers), and these positive others thus facilitate healthy selfobject experiences. For Kohut, the self and its corresponding selfobjects resembled the relationship between the human body and oxygen in the atmosphere; oxygen is essential, however, it is mostly plentiful and lungs are usually healthy. Consequently, respiration typically goes on, stress free, unnoticed, unappreciated, and without concern (Lessem, 2005).

Speaking developmentally, Kohut noted that an infant is prepared with certain innate mechanisms (temperament, biological needs, physiological capacities), but with an unformed sense of self. This sense of self was believed to grow as the child's innate mechanisms began to interact with the parents' responses to the child's selfobject needs, as certain abilities and expressions were reinforced, while other were not. Kohut (Kohut\& Wolf, 1978) theorized that during the second year of life, a core sense of personality would begin to form.

It is perhaps in the relationship between infant and parent that the selfobject experience is most clearly seen. Kohut (Kohut \& Wolf, 1978) clearly detailed that it was not merely the parents' actions, but perhaps more importantly, the parents own state of being: "Their selfconfidence as they carried us when we were babies, their security when they allowed us to merge our anxious selves with their tranquility... will be retained by us as the nucleus of...strength (p. 417)." 
Speaking more generally, the care provided by a parent or caretaker was argued to provide the child with the beneficial selfobject experiences, which ultimately leads to a cohesive sense of self. For example, when a mother provides consistent soothing and nurturing for a child, the resulting selfobject experiences provides the scaffolding through which the child's capacity for self-organization is developed and maintained, resulting in a sense of self. This sense of self, when it achieves a sense of consistency and cohesion, brings with it a sense of selfesteem (Wolf, 1988). Though these early selfobject experiences were theorized as both critical and foundational, the need for selfobjects will remain present throughout life.

\section{Selfobject Needs}

Kohut's (1971) position that selfobject functions are necessary for both the development and maintenance of one's sense of self (and resulting psychological health), almost necessarily leads one to question what specific selfobject needs there are. Kohut (1977) postulated a tripolar self: (a) a desire for recognition and power, (b) a need for idealized goals and idols, and (c) a need for kinship and bonding. Corresponding to these selfobject needs, Kohut posited(a) "the need for establishing and maintaining self-esteem (mirroring)," (b) "the need to feel safe and to have the capacity to self-sooth (idealizing)," and (c) "the need to experience and friendship and connection (twinship)."

Following along with Freud's (1914) earlier concept of primary narcissism, Kohut believed that an infant initially perceives itself as inseparable from the mother, and this unity is necessary because the infant has yet to develop an independent sense of personality. With time and increased interaction, the process of developing a sense of self begins, and the child begins to feel the sense of complete unity with the mother erode. In response, the child attempts to secure this sense of unity by drawing upon two images: the grandiose self and the idealized 
parental "imago" (Kohut, 1971). In the case of the former, the child is seen as wonderful and powerful, while in the case of the latter, the parent is seen as a source of perfection. These processes allow the child to develop a cohesive sense of self through a merger of the grandiose self and the idealized parental image. The grandiose sense of self is ultimately transformed into healthy ambitions, while the idealized parent imago becomes the child's internal values and ideals. However, if these needs are not met, the images remain in their primitive form (i.e., do not develop into their mature and healthy forms), and the child's sense of self becomes vulnerable, leaving the child to struggle with the maintenance of self-esteem and self-cohesion.

As a matter of historical consideration, it is important to note that Kohut initially posited the existence of a bipolar self, elaborating only the mirroring and idealizing selfobject need, with twinship being adding later. Similarly, other self psychologists have posited a number of selfobject needs beyond Kohut's initial formulation, including: self-delineating need (Trop \& Stolorow, 1992), efficacy need (Wolf, 1988), validation need (Stolorow, Brandchaft, \& Atwood, 1987), and the adversarial need (Wolf, 1988). However, the three selfobject needs posited by Kohut, mirroring, idealizing, and twinship, have received the greatest attention, and will be the ones explored in the present study.

Mirroring Selfobject Need

Kohut (1977) was profoundly impressed by the importance of young children's desire to revel in their accomplishments and to have this satisfaction shared by their parents and caregivers. As conceptualized by Kohut, the child has the desire to achieve something, to enjoy a sense of pride related to their accomplishment, and to then have the parents mirror that sense of pride. It is thus in this desire of the child to capture the "gleam in the mother's eye" that the mirroring selfobject is named. 
In its most fundamental form, the need for mirroring was believed to reflect the child's need to have its sense of worth and value both recognized and celebrated. A common example of such mirroring may be seen in the child presenting to the parent a new effort or skill. The child yelling to its mother, "Look what I can do!" is not merely requesting attention, but is seeking confirmation of its own special nature. Consistently receiving such positive acceptance and recognition of its own worth will allow the child to internalize a sense of self-esteem, thereby providing grounds for a sense of vitality and worth (Silverstein, 1999). As mentioned previously, such mirroring will ultimately be internalized as a person's sense of goals and ambitions as they move towards adulthood.

Kohut argued that a lack of mirroring experiences, or having mirroring experiences which are consistently painful, can have important psychological implications. Often children respond by striving to develop a sense of self that is "worthy" of the parents' admiration, tacitly accepting that something about them is wrong or lacking. These children seek to address their perceived shortcomings by excelling in an area which will gain the parents' favor (such as being sweet or athletic). Conversely, other children will deny the aspects that such mirroring experiences would develop, such as pride or a sense of agency. This denial ultimately proves problematic, however, as these disavowed feelings remain, thereby creating a sense of internal conflict (Lessem, 2005). In either case, such children are theorized to develop a sense of self that is ill-prepared to withstand psychic injury, ultimately resulting in feelings of apprehension, moodiness, or feeling unfocused, a state which Kohut described as "fragmentation" (this may be colloquially described as "falling apart").

A common pathological response to repeated painful or lacking mirroring experiences was described by Kohut (1971) as the "vertical split." Kohut described the vertical split as a 
kind of dissociative process wherein the child is thought to cast aside the early narcissistic fantasies, and instead seeks to craft a persona based upon the parent's needs and wishes. In this fashion, a false sense of self receives the craved mirroring, while the child's own desires and needs are neglected. The child is thereby believed to become trapped by the false sense of self. Because the child's own needs and ambitions have become disassociated from the rest of its life, they cannot add to the child's psychic structure by transforming into positive ideals and healthy ambitions for the child to strive for. Nonetheless, these feelings exert a powerful influence on the child. The child may grow to present as cold and arrogant, but without the mirroring selfobject experience from which to draw self-esteem, the child's self-esteem is burdened with a sense of vulnerability. The child may ultimately grow to be hypersensitive and fragile, reacting to perceived slight with shame and rage (Summers, 1994).

Repression (the unconscious defense denying one's own desires) may also provide a response to insufficient mirroring experiencing, a process Kohut described as the "horizontal split." In this instance, the child is theorized to experience a sense of disapproval or rejection in the face of their efforts to obtain mirroring. To prevent the painful experience of such empathic failures, the desires for mirroring are repressed. This affective repression is sometimes described as a form of narcissistic depletion, characterized as a sense of emptiness or worthlessness. Kohut noted that such individuals often experience chronic boredom and dissatisfaction, as the early sense of narcissistic grandiosity is unfulfilled, robbing much of their experience of its potential delight.

\section{Idealizing Selfobject Need}

Just as the child requires of the admiration of the parent, Kohut also described the child's need to idealize the parent. In this process, the child seeks to identify with an agent more 
capable than himself/herself. Although the child needs to have his/her own sense of power, s/he also looks to the parents as beings of enormous strength, a kind of idealized role model. In theory, the child begins to view the parent as an invincible and idealized figure, and this admiration of the parent will ultimately enhance the child's sense of self. Thus, the child derives an enhanced sense of worth through his/her connection with the parent through something of an unconscious syllogism: "You are a powerful being, I am part of you, and therefore I am powerful too."

In times of fear and pain, a child can experience the threat of self-fragmentation, and idealization of a parent can prove greatly restorative. In perhaps the most classic instance of this, one might consider the case of a child who falls and scrapes his knee. The child responds with fear and panic, and yet, the kiss of a mother can offer a soothing balm. In Kohut's view, the child draws strength through his connection with the mother, who has the incredible power to “make everything better."

When Kohut discussed the idealizing selfobject, he had in mind two particular functions: a calming, soothing function, and an idealized caregiver and protector. In the case of the former, the crucial ability is that to sooth the child, much as a mother's voice can sooth a young infant. The latter refers to the child's growing sense that the world is a threatening and confusing place at times. As the child is forced to face these threats, it can be a source of great comfort to know that a supremely powerful being is there to assist and protect them. "My dad is the strongest man in the world," a child may boast, and indeed, there is obvious value in having such an ally on your side.

As described by Kohut, with this idealized image comes a sense of safety, allowing the child to better confront outside challenges and internal anxieties. Thus, in an ideal sense, the 
parent must accept and accommodate the child's idealizing needs (these needs will of course develop and mature). If, however, the child is not allowed to have such feelings, or if the parent fails the child in some fundamental way, a number of negative consequences can arise. First, the proposed sense of safety may not develop, leaving the child ill-prepared to confront feeling of fear, anxiety or depression. Second, the child is deprived of an idealized image to strive to meet, an internal idea that the child might desire to become. Children without such ideals may find themselves without a sense of value or purpose, or may question their capacity to succeed in the world. Finally, the child may experience a sense of being incomplete, as there remains an unfulfilled desire to connect with some idealized other.

\section{Twinship Selfobject Experience}

In Kohut's early (1971) formulations, he argued that personality was best conceptualized as a "bipolar self." The bipolar self was characterized by a desire for power and success on one hand, with personal goals and values on the other. The person's talents and abilities stood as a link between these two aspects of the self, and a sense of self would grow as the person employed their abilities to become successful and reach personal goals (Carducci, 2009).

However, Kohut's (1984) last work would offer a third selfobject experience, as Kohut's clinical work suggested the presence of a third selfobject need. Kohut thus advanced his theory to one of a tripolar self. This final selfobject experience was referred to as a twinship need, which Kohut had initially described as a component of the mirroring transference. Kohut described twinship as the need to experience a fundamental sense of "alikeness" from the world around us, a sense of belonging and participating. It serves as a confirmation that one belongs with others, that one is part of the group, as opposed to a solitary and separate individual. Following from such unity, Kohut posited, was a sense of self-worth and identity. 
Kohut described the twinship need as active throughout life, beginning with a more primitive desire for merger, as the child powerfully experiences the other as part of itself, disallowing for the other's independent existence. As the need grows more sophisticated, tolerance of the individuality of others develops, and individuality and connectedness are viewed as congruent. Kohut offered the example of a young boy standing beside his father while his father shaved as an early example of twinship. The young boy is not merely imitating the father's behavior, but attempting to confirm his standing as connected and similar to the father. It is the attempted connection, and not the behavior that is critical. A child who whispers their fears to beloved teddy bear is seen to be engaging in the same process. It is not the object or the specific action that is important, but replication of one's own experience in another, even if said other is a figment of one's imagination (Silverstein, 1999). ${ }^{1}$

As the child ages and enters the oedipal phase, the need for twinship was theorized to become increasingly important as a means for gender identification (Basch, 1992; Kohut, 1984). The process of solidifying one's gender identity is greatly assisted through the experience of twinship with one's same-sex parent. However, it is important to note that the twinship need described by Kohut is not merely an attempt to mimic behavior, but rather, a means to experience a sense of belonging and connection. The fulfillment of this need, and not the external behavior, is the key matter of Kohut's focus.

As the child moves into adolescence, Kohut believed that the experience of twinship with members outside of the immediate family became crucial. The child's sense of identity was

\footnotetext{
${ }^{1}$ Much confusion about self psychology arises from use of the term "object," as opposed to "person." The term object is employed because abstract concepts, symbols, or even imaginary friends can fill the role of a selfobject. Indeed, the notion of a religious figure filling such a role appears immediately plausible. However, such beliefs would have to be deeply held in order to function in such a capacity.
} 
theorized to become clearer, in part through an increasing contrast with the family unit. Peer group norms are often accepted without question, as the child's taste in music, clothes, hobbies and the like may in no small part be derived from perceived social norms. Indeed, the child may have significant fear regarding deviations from such norms, both as a matter of social consequence, or the belief that it might reflect a certain oddity or defect in their character. Successful twinship experiences during this time provide the child with gentle reassurance and a sense of belongingness, both of which can offer feelings of validation as the child works to craft his or her identity.

Moving into adulthood, Kohut believed, the experience of fraternities, military service, becoming supporters of a local team, or even proudly displaying one's city of origin provide a means to seek twinship experiences. These twinship experiences provide an important reminder

of our fundamental sameness and connection with others, a bastion against the notion of isolation.

Should twinship needs be significantly disrupted or chronically unmet, individuals can experience a profound sense of disconnect and isolation. Such persons might feel unable to connect with social groups or co-workers, instead feeling largely alienated. In some instances, this can also represent an inability to connect with the group's larger values, further isolating the individual.

Selfobject Needs as a Consistent Component of Mental Health

A fundamental component of the self psychology articulated by Kohut was the premise that the need for selfobject responsiveness is never outgrown. People require the interaction of others to maintain their sense of self, and lack of such experiences can prove to be harmful at any stage of life. What changes, Kohut posited, was the maturity of the selfobject needs. As an 
infant, such selfobject needs prove all-encompassing, much like the infant is initially utterly dependent upon the mother. As the child grows older, it may seek selfobject experiences from a variety of sources, be they friends or loved ones, or even abstract values and religious beliefs (Baker \& Baker, 1987; Hahn, 1994).

In the ideal path of development, the self is nurtured through the selfobject experiences of mirroring, idealizing, and twinship, or what Kohut (1984) referred to as the constituent components of the self. These constituent components of the self would in turn give rise to the self's attributes: cohesiveness (an enduring experience of the self as whole), vigor (feeling empowered to face the world), and harmony (a capacity to soothe oneself).

In the course of normal development as conceived by Kohut, consistent selfobject experiences led to the development of healthy attributes, and ultimately, a healthy sense of self. Kohut believed that a healthy sense of self was manifested in a number of critical ways (Teicholz, 1999). Perhaps most importantly, it allows individuals to connect empathically with others, as well as to experience and express intense positive emotions. According to Kohut, such connections and emotional experiences greatly strengthened the self. Further, it instilled in one the confidence to approach and attempt new tasks, ultimately resulting in a greater sense of achievement, which would in turn lead to greater self-esteem (Lessem, 2005). Finally, it allowed for a continuous process of growth, as the individual was provided the strength to seek to reach the potential of his or her ideal self, and to continue to internalize the idealized image of admired persons.

Yet just as the self remains ever-dependent upon selfobject experiences, it was further hypothesized to remain vulnerable to their absence or insufficiency. Although such vulnerability is more pronounced during the early years of childhood, there is also a heightened vulnerability 
during transitional phases as well, such as adolescence, marriage, and parenthood (Wolf, 1988). In the case of a generally healthy adult, compensatory mechanisms might alleviate the distress brought on by inadequate selfobject experiences, however, early in development, no such compensatory mechanisms exist.

Kohut's general assertion was that troubled interactions between the child and the parents may often represent painful selfobject experiences, and to the developing self such experiences can be damaging, particularly if they reflect a general trend. In the face of such selfobject experiences, the child's sense of self may be damaged, particularly regarding one of the constituent components of the self. This disruption of the child's developing sense of self can have significant consequences, and the noted attributes of the self cannot develop (instead leaving one to feel fragmented, weak, and unable to soothe one's own emotions). Speaking in terms of a more typical clinical language, an individual becomes predisposed to feelings of depression, anxiety, anger, rage, and shame. Kohut (1984) argued that these symptoms arise from the state of internal tension that exists due to a lack of self-cohesion (i.e., a lack of security and stability). Kohut thus considered disruptions of self-cohesion and diminished self-esteem as the primary psychopathology, with ego deficits and weaknesses as defensive reactions one's unstable self-cohesion.

\section{Selfobject Experiences as the Core of Treatment}

A full discussion of the treatment approach applied in self psychology is beyond the scope of the present work. However, it is important to note that addressing selfobject needs is the core of the self psychological treatment. The basic goal of treatment is to identify empathic disruptions that occur within the treatment, and to further examine the emotional consequence such disruptions carry. As the patient grows increasingly aware of this pattern, the insights 
offered by the therapist allow the patient to bolster their ailing sense of self, simultaneously pacifying their clinical difficulties. Kohut described this process as "transmuting internalization."

In the course of treatment, the patient is expected to develop a selfobject transference towards the therapist. Inevitably, this transference will be disrupted by an empathic failure on the part of the therapist, much as Kohut experienced in the case of Ms. F. The empathic failure is experienced as hurtful by the patient, and further connects with the early empathic failures of the patient's caregivers. In other words, the painful selfobject experiences of childhood are reawakened. However, the process is often not so straightforward, as the patient may present with defenses in the course of therapy, as these defenses are used to protect oneself against the painful re-experiencing of early selfobject failures. Returning to the case of Mr. Taylor, his use of the defense of projection (attributing unacceptable internal feelings to an external source, such as another person) is evident in his description of the social skills group's pathology. .

The therapist is expected to note such occurrences, as they are believed to provide insight into the state of the patient's self-cohesion, and may help in revealing the constituent need that has been neglected. The therapist conveys this analysis to the patient, including the perceived etiology of the patient's reaction, as well as the importance of noting the patient's frustration. By consistently interpreting the in-treatment responses to the therapist's empathic failures, the patient is intended to construct a revitalized sense of self (Silverstein, 1999).

The reparative agent in the treatment is believed to be the repeated identification of disruptions in self-states. As the selfobject needs are persistently identified, transmuting internalizations are gained. The formerly stalled developmental process is reignited in an empathic environment, allowing for the repair of injuries to the self, and the growth of self- 
esteem. Through this, Kohut argued, the idealizing transference is built into the superego (i.e., an individual's sense of beliefs and ideals), while the grandiose transference is channeled into the development of ambitions and desires.

The constructs outlined by Kohut $(1971,1977,1984)$ present an interesting challenge for potential researchers. It is perhaps therefore unsurprising that there have been multiple case studies involving the theory, but there has been only minimal empirical research. However, an important exception to this lies in the study of Narcissistic Personality Disorder, in which multiple studies have offered evidence to support Kohut's theories. In the following chapter, the theory and research behind the study of narcissism is reviewed.

\section{Summary Paragraph for Chapter I}

Chapter I presented a review of Heinz Kohut's theory of self psychology, the theory that is the subject of the present dissertation. Kohut argued that the sense of self represents the core of one's s personality, and in the course of normal development, a healthy sense of self provides one with a feeling of strength and vitality. Thus, while we all undergo stressful experiences and challenges, a healthy sense of self allows us to respond to these difficulties while maintaining emotional and behavioral control. However, our sense of self, as well as our emotional strength, requires the support of other people. People around us bolster our sense of self by strengthening, or filling in for, our natural restorative capacities. In other words, we need other people, and Kohut described specific needs, called selfobject needs, that people in our life provide. For children, these selfobject needs are particularly important, as their sense of self is still developing. Kohut stated that there were three primary selfobject needs that a child requires throughout development (and beyond): (1) A need to view one's parents/caretakers as strong and powerful people that the child can look up to - Idealizing; (2) A need to have one's own 
abilities and accomplishments be appreciated by parents/caretakers - Mirroring; and (3) the need to see oneself as fundamentally connected and similar to other people - Twinship. The present study is designed to create a measure capable of assessing the degree to which a person experiences these three needs as being unmet, which, according to Kohut, results in psychological health. . 


\section{CHAPTER II}

\section{THE THEORY AND STUDY OF NARCISSISM}

\section{Narcissistic Personality Disorder}

Although much of Kohut's work has received scant empirical attention, an important exception to this observation is found in the study of the Narcissistic Personality Disorder, a subject of great interest to Kohut. The concept of the Narcissistic Personality Disorder has existed in some form since antiquity, as it roots may be traced ancient Greek mythology. Indeed, Ovid's tale of Narcissus clearly describe the arrogance, self-infatuation, and callousness that is readily seen in the narcissistic pathology as described in contemporary mental health texts. Furthermore, the concept has been considered in psychoanalytic theory for over a century.

Yet despite this historical interest, narcissism was not recognized as an official mental disorder until the publication of the Diagnostic and Statistical Manual of Mental Disorders $\left(3^{\text {rd }}\right.$ ed; DSM; American Psychiatric Association [APA], 1980). The inclusion of Narcissistic Personality Disorder in the DSM-III the inclusion of NPD in the DSM was a significant development, as it allowed for an increase in empirical investigations into the construct. The DSM-III criteria were used to create objective empirical methods of measurement, most notably the self-report, Narcissistic Personality Inventory (NPI; Raskin \& Hall, 1979). Armed with the NPI, a number of researchers began investigations into the narcissistic disorder (e.g., Raskin \& Terry, 1988; Wink \& Gough, 1990).

Though the DSM conceptualization and the creation of the Narcissistic Personality Inventory offered important developments in the understanding of narcissism, a number of theorists soon objected that the narcissistic description provided by the DSM was too narrow to capture the full scope of narcissistic personalities (Balestri, 2000). Many argued that the DSM 
had mistakenly focused on overt qualities of narcissism (e.g. exploitive tendencies, arrogance, inflated sense of importance, and demand for admiration) while neglecting the disorder's more covert features (e.g. vulnerability, feelings of shame, shyness). Essentially, it was argued that the characterization of narcissism as reflecting obvious and unbridled arrogance was incomplete. While such a description was indeed fitting for certain patients, considerable clinical data indicated that many narcissistic patients evidenced grandiose fantasies in concert with a selfeffacing and insecure demeanor. These objections, and the resulting debate within the literature, has come to be known as the Kohut/Kernberg controversy.

\section{Kohut versus Kernberg}

Historically speaking, narcissistic pathology was described as a form of maladaptive object relations $^{2}$ (Freud, 1914). Freud (1914) suggested that narcissism resulted from the libidinal cathexis of the ego,(i.e., the transfer of the libido from the object to the ego). In other words, directing one's emotional energy inward instead of outward (Freud (1914)would elaborate this process with his U-tube analogy). According to Freud's understanding, infants began life in a phase wherein the world is experienced as part of the infant itself (he described this phase as autoerotic primary narcissism). In the correct developmental process, one would advance beyond this autoeroticism and ultimately develop a capacity for object love. In this state of object love, libidinal energy was directed outward, toward loved objects. Thus, according to Freud, narcissism was something to be outgrown (even though everyone was believed to retain some elements of narcissism). Freud further described narcissism and object love as inversely related, such that in order for object love to grow, one's narcissism had to diminish. In the case

\footnotetext{
${ }^{2}$ In brief, object relations is a psychoanalytic school of thought that emphasizes the importance of early developmental experiences for determining the character and style of adult interactions. Interestingly, it shares a good deal in common with self psychology.
} 
of Narcissistic Personality Disorder, because libidinal energies were invested in the ego, they were in turn withdrawn from others. Self-involvement thus grew at the expense of the capacity to love other people, resulting in the selfishness and exploitative nature found in narcissism.

Like Freud, Kernberg (1970, 1974a, b, 1988) believed that narcissistic patients needed to overcome their excessive self-love in order to develop their capacity for object love. Kernberg argued that narcissistic grandiosity reflected a pathological process in development, its etiology connected with the narcissistic patient's difficult childhood. Kernberg stated that in the prototypical example of a narcissistic patient's development, they were confronted with cold and antagonistic parents. The parents' behavior toward the child alternated between a marked indifference and an undercurrent of aggression and resentment. Nonetheless, the child was typically assigned a special role within the family dynamic (such as being the perfect student). The child would attempt to fall back upon this special position as a way to shield itself against the parents' negativity, but this approach would prove untenable, ultimately leading to significant psychopathology. After repeatedly enduring the onslaught of the parents, the child would soon be left with the retreat to the "special position" as the only remaining defense. To escape the reality of the rejecting parents, the child would learn to regard its "special position" as a way to split itself from reality (i.e., the uncaring parents). Rather than integrating positive and negative representations of the self, the child internalizes only the positive and idealized facets of itself and of the external objects in the environment. The child simultaneously "splits" itself (i.e., dissociates) from the negative characteristics of the self and projects them onto others. ${ }^{3}$

\footnotetext{
${ }^{3}$ The contrast here between Kohut and Kernberg is significant, but easy to overlook. Kohut described the self as fundamentally nondefensive, while Kernberg viewed narcissistic grandiosity as a specific and aggressive defense. Kernberg emphasized the aggressive component of narcissism, arguing that it was a primary concern of the
} 
The result of this process, according to Kernberg, is an arrogant, aggressive, and overtly grandiose individual. This more severe description of the narcissistic personality was not unintentional, as it resulted from Kernberg's belief that the narcissistic individual's defensive organization is highly similar to that of the borderline personality disorder (a personality style characterized by instability of mood, interpersonal relationships, and self-image). What distinguished the narcissistic individual was his grandiose, but nonetheless integrated, core sense of self (Kernberg theorized that this sense of self was formed by a merger of the ideal self, the ideal object, and the real self into one entity). Thus, although narcissistic individuals repeatedly employ primitive defenses (e.g., splitting, omnipotence) in a manner similar to the borderline personality, they tend to have a consistent and relatively functional self-structure (i.e., a consistent sense of self that is described as lacking among those diagnosed with borderline personality disorder).

In sum, Kernberg's fundamental premise is that the narcissistic individual identifies with an idealized self-image in the hope of severing any dependency on other people (i.e., external objects) as well as the inner images of those objects (Gabbard, 1983). Furthermore, narcissistic individuals deny the existence of personality traits that would conflict with their idealized selfimage by projecting them onto others. Consequently, such individuals not only see themselves in an idealized fashion, but also tend to be dismissive of the needs of those around them (both because they seek to sever their dependency on others, and because they have projected their own negative characteristics onto said others).

The contemporary understanding of the Narcissistic Personality Disorder still relies

narcissistic pathology. Kohut's description emphasized the experience of vulnerability, with aggression arising as a natural response to parental failures. 
heavily upon the explanation offered by Kernberg (1970). Indeed, the influence of this early analyst is clearly seen in the current DSM criteria. Goldstein (1985) went so far as to contend that the current DSM criteria represent a summary of Kernberg's ideas, an opinion seconded by various other researchers (e.g., Millon, 1981). Yet it is this perceived allegiance to Kernberg's description of narcissism that has been the source of great scrutiny. Soon after the publication of the DSM-III, several theorists (e.g., Cooper, 1988; Gabbard, 1983; \& Philipson, 1985) criticized the DSM and its narrow depiction of the narcissistic disorder, noting that while the criteria were adequate for diagnosing the more obvious symptoms of the overt narcissist, the symptoms of the shy and inhibited narcissist were not readily encapsulated. A consideration of Kohut's description of the disorder, they noted, was missing.

Although Kohut did not deny the existence of the narcissistic individual as described by Kernberg, a number of his patients did not demonstrate the typical symptom cluster associated with the narcissistic pathology. He noted that although some of his patients appeared to have grandiose fantasies and ambitions, these individuals were also plagued by a highly fragile selfesteem that would experience great injury if they sensed a hint of derision from those around them. The brash and aggressive form of narcissism as described by Freud did not seem capable of describing the inner workings of these particular patients, and in response to these deficiencies, Kohut would develop a radically different conceptualization of the narcissistic pathology.

Kohut strongly objected to the highly pejorative view of narcissism advanced by Freud. Rather than characterizing narcissism as an early state of selfishness that needed to be discarded, Kohut conceptualized narcissism as a core source of creativity and power that if properly nurtured, could result in a stable sense of self and a corresponding sense of self-esteem and 
energy. As a matter of fundamental difference, Kohut strongly opposed Freud's belief that narcissism and object love were inversely related. Indeed, Kohut's notion of the selfobject spoke to his belief in the importance of interdependence between self and other. In the form of mature narcissism, he posited, one grows to accept this sense of interdependence between self and other (i.e., the continued need for selfobjects). Independence was not the sign of mental health, Kohut argued, rather, it was the ability to have one's selfobject needs met in an increasingly diverse number of ways.

Kohut further distinguished himself from Freud and Kernberg with his notion of narcissism having in its own course of development, distinct from the development of object love. Kohut posited that object love and narcissism were complementary, though separate, developmental axes. As detailed previously, Kohut asserted that adequate selfobject experiences allowed the grandiose sense of self to ultimately transform into healthy ambitions, while the idealized parent imago would eventually becomes the child's internal values and ideals. These two developmental lines, according to Kohut, existed on a separate axis from that of object love.

Perhaps most divergently, Kohut articulated a view of narcissism as existing on a continuum, ranging from healthy to pathological. With the development of healthy narcissism, there is a cohesive sense of self, and one is able to act with confidence and a strong sense of selfworth. Further, in response to disappointment, criticism, or failure, the individual still experiences pain and frustration, but is able to self-soothe and ultimately recover.

Conversely, Kohut believed that the pathological form of narcissism was evidenced by extreme self-preoccupation, as well a lack of self-cohesion. Such individuals experience great fragility in their sense of self-esteem, as well as a largely unstable self-concept. Kohut described narcissistic individuals as often finding themselves at the mercy of feelings of shame or 
humiliation, and in response, Kohut posited that they craft a defensive shell to protect themselves. And it is through this defensive response that grandiose fantasies of self-importance arise, as does the need to see other people, not as autonomous individuals, but as a tool to have one's own needs met (much in the way Kohut experienced Miss F's style of relating to him). This fragility, in combination with the need to treat others as a means to have one's own needs met, can often result in a sense of interpersonal distance and isolation.

\section{Covert versus Overt Narcissism}

The heirs to this debate have argued for a wide range of narcissistic presentations (Gabbard, 1989; Hibbard, 1992; Shulman, 1986; Wink, 1991), however, two primary forms of narcissism have grown to be increasingly accepted: the covert narcissist (reflecting Kohut's description) and the overt narcissist (reflecting Kernberg's description).

Gabbard (1983) offers a particularly thorough discussion of the two types, describing the overt narcissist as oblivious, while noting that the covert narcissist is best described as being hypersensitive. Overt narcissists act with no apparent awareness of how their actions are affecting those around them, often dismissing the objections and concerns of others. Their sense of self-focus is manifest in a multitude of ways: rather than engaging others in conversation, they tend to speak as though they were addressing a crowd (e.g. rarely make eye contact); their conversations tend to be thoroughly self-promoting, as they happily list their various accomplishments and attributes; and they express little interest in conversations that are not focused on their own concerns. Such overt narcissists demonstrate an apparent need to be the center of attention, and they are seen by others as overbearing and selfish. As Gabbard notes, descriptions of the overt narcissist are largely reflective of the DSM criteria for Narcissistic Personality Disorder. 
The covert narcissist, conversely, presents in a very different fashion. These individuals are highly sensitive and are acutely aware of the reactions of other people. In contrast to the selfabsorption of the overt narcissist, covert narcissists carefully direct their attention outward, vigilantly monitoring other for signs of disapproval. This kind of narcissist is typically quite shy and may often be highly self-critical. S/he expresses great fear over being the center of attention, worrying that such scrutiny will be met with humiliation and rejection. Though they have the same dreams of grandiosity that the overt narcissist expresses, the covert narcissist experiences a sense of shame related to these fantasies.

Though both the covert and overt forms of narcissism struggle with the maintenance of their self-esteem, the methods they adopt are notably different. Overt narcissists freely express their grandiosity in an attempt to impress those around them. That their audience may not be interested in their boasting is irrelevant; the overt narcissist successfully ignores the critique that others would offer. Covert narcissists, however, maintains self-esteem by avoiding situations in which they must function as the center of attention, instead offering a shy and reserved demeanor. Thus, both forms of narcissism wish to see themselves as worthy of admiration, possessing a worth beyond that of a normal person. Yet, while overt narcissists freely express their arrogance, the covert narcissist worries that others will react negatively if they embrace their immodesty. They believe that their shameful appraisal of their fantasies is shared by others and thus act to hide them under a veil of insecurity.

\section{Empirical Research Supporting the Covert/Overt Distinction}

In an early effort to provide an empirical demonstration of the narcissistic personality disorder criteria, Ronningstam \& Gunderson (1990) administered the Diagnostic Interview for Narcissism (Gunderson, Ronningstam, \& Bodkin, 1987) to 82 psychiatric patients. This measure 
includes five domain of function, including elements both within and beyond the DSM criteria: grandiosity, interpersonal relations, reactiveness, affects and moods, and social and moral adaptation. Of the 82 participants, 24 were identified as having narcissistic personality disorder, while the remainder reflected a range of psychiatric conditions. During these interviews, traits common to the narcissistic patients were identified and categorized. As expected, qualities such as perceived superiority, exaggeration of one's own abilities, boastfulness, grandiose fantasies, selfishness, and demand for admiration were all found among narcissistic patients. However, the researchers noted other traits suggested by Kohut (1971) were present in both the narcissistic group and the comparison groups. Among these traits were hypersensitivity, need for approval, and sensitivity to criticism. Gunderson et al., (1990) concluded that the DSM criteria failed to capture the disorder in its totality and that an over-reliance on the DSM caused some patients with narcissistic conditions to become misdiagnosed.

A number of subsequent investigations have considered this distinction, and increasing evidence has bolstered Ronningstam and Gunderson's (1990) conclusion that the narcissistic disorder as presented in the DSM is incomplete. The existence of a type of narcissism as described by Kohut has arisen in numerous empirical studies, though the language describing this second type of narcissism has been highly variable. For the present investigation, we draw upon the terminology employed by Paul Wink, one of the most prolific researchers on the matter. Wink (1991), building upon the recognition that two of the most common measures of narcissism, the Narcissistic Personality Inventory (NPI; Raskin\& Hall, 1979) and the Narcissistic Personality Disorder Scale (NPDS; Ashby, Lee, \& Duke, 1979), advocated for a distinction of what he described as covert (i.e., a narcissist similar to what Kohut described) versus the overt (the narcissistic typology advocated by Kernberg) narcissist as described by the DSM. 
In an early investigation of the covert/overt distinction, Wink (1991) performed a principal component factor analysis of six MMPI scales designed to measures narcissistic pathology: Raskin and Novacek Narcissism Scale (Raskin \& Novacek, 1989), Morey, Waugh, and Blashfield Narcissism Scale (Morey, Waugh, \& Blashfield, 1985), Wink and Gough Narcissism Scale (1990), the Narcissistic Personality Disorder Scale (NPDS; Ashby, 1979), the Ego Sensitivity Scale (ESS; Pepper, 1985), and the Narcissism-Hypersensitivity Scale (Serkownek, 1975). The investigation identified two orthogonal factors, labeled Vulnerability/Sensitivity and Grandiosity/Exhibitionistic, Wink referred to them as covert and overt, respectively. The latter was comprised of the DSM-based measures, while the former represented the scales derived from the Masculinity/Femininity scale. Elevations on either factor were associated with qualities of self-indulgence, arrogance, and a tendency towards envy. However, the two also demonstrated significantly different behavioral correlates. The covert factor was found to be positively correlated with traits of introversion, anxiety, hypersensitivity, and defensiveness. Conversely, the overt factor was positively correlated with the traits of exhibitionism, extraversion, self-assuredness, and aggressiveness.

In a subsequent study, Wink (1992) utilized a Q-set rating of narcissistic types, in addition to the factor-analytic methodology previously detailed. In a study of college women using a Q-methodology, Wink identified three forms of narcissism, which he labeled as willful, hypersensitive, and autonomous. The willful narcissist appeared to parallel the overt narcissist, displaying exhibitionistic tendencies, grandiosity, and high levels of confidence. The hypersensitive narcissist paralleled the covert narcissist, appearing introverted, inhibited, and restrained, though with a hidden core of grandiosity. However, the use of Q-methodology also suggested the presence of a well-adjusted and healthy narcissist, as it was unrelated to measures 
of pathology, while being positively correlated with traits of self-assurance and confidence (providing support for Kohut's notion of the developmentally adaptive qualities of narcissism).

In a study highly similar to that of Wink (1991), Rathvon \& Holmstrom (1996) examined the covert/overt distinction using the updated version of the MMPI-2. In this analysis, the Narcissistic Personality Inventory and the following five scales were subject to a principal components factor analysis: Pepper and Strong's Masculinity/Femininity (Mf) Ego Sensitivity subscale (PS; Pepper \& Strong, 1958), Serkownek's Mf Narcissism-Hypersensitivity Scale (SHNS; Serkownek, 1975), the Narcissistic scale of Ashby, Lee, \& Duke's Narcissistic Personality Disorder Scale (ALD; Ashby et al., 1979), Morey, Waugh, and Blashfield's Narcissistic scale of the Personality Disorder Scales (MWB; Morey et al., 1985), and Wink and Gough's Narcissism Scale (WGNS (Wink \& Gough, 1990). As in Wink's 1991 study, two orthogonal factors emerged, one reflecting themes of grandiosity, and the other reflecting a sense of depletion. The WGNS, Narcissistic Personality Inventory, and the MWB scales loaded .78, .83 , and .86 on the Grandiosity factor, while the ALD, PS, and the SNHS scales loaded .84, .89, and .93 , respectively, on the Depletion factor. With the exception of the WGNS, which demonstrated a factor loading of .40 on the Grandiosity factor, there were no significant crossloadings between the factors. These factors were further characterized by their MMPI profiles. The Narcissistic Grandiosity factor was found to positively correlate with scale 9 (Mania, Ma), and those with high scores on the grandiosity factor typically had average F scores and were equally well-described by $98 / 89$ or $96 / 69$ codetypes. Conversely, the Depletion factor was found to positively correlate with scale 2 (Depression, D), scale 7 (Psychasthenia, Pt), and scale 0 (Social Introversion $\mathrm{Si}$ ), and those high on the depletion factor typically demonstrated elevations on the F scale and were best described solely with a 98/89 codetype. 
Hibbard (1992) found evidence of the covert/overt distinction in an analysis of 701 college students. All participants completed eight scales measuring narcissism, object relations, masochism, social desirability, and shame. A factor analysis identified two factors consistent with the overt/covert distinction: a grandiose style and a narcissistically vulnerable style. Those identified as demonstrating the vulnerable style demonstrated a significant correlation with disruptions in object relations and masochism, suggesting that the vulnerable group experienced a greater level of core pathology. However, the core difference between the two styles appeared to be based upon the experience of shame, correlating negatively with the grandiose style, and correlating positively with the vulnerable style. Further, this relationship was not mediated by social desirability. The study, Hibbard (1992) concludes, provides further evidence for the covert/overt distinction.

Reviewing the literature focused upon the range of narcissistic presentations, Wink (1996) found further evidence to support his overt/covert distinction. Summarizing the results of several studies, Wink (1996) identified a disparate pattern of correlations between the covert and overt forms of the narcissistic pathology. Wink first selected three measures derived from the DSM-III criteria, all of which were theorized to assess the overt form of narcissism: the Narcissistic Personality Inventory (Raskin \& Hall, 1979), and two MMPI-based scales. Scores on these measures were found to positively correlate with traits such as ego-expansiveness, selfcenteredness, assertiveness, and impulsivity (Raskin \& Novacek, 1989; Raskin \& Terry, 1988; Wink \& Gough, 1991). Wink (1996) then selected three measures of covert narcissism -- the Narcissistic Personality Disorder Scale (Ashby, 1979), the Narcissistic-Hypersensitvity Scale (Serkownek, 1975), and the Ego-Sensitivity Scale (ESS; Pepper, 1985), and identified the traits most commonly associated with them. These measures demonstrated strong positive correlations 
with traits such as sensitivity, depression, anxiety, and fears of social inadequacy (Graham, Schroeder, \& Lilly 1971; Wink, 1991).

A further study of the covert/overt distinction was conducted by Wink and Donahue (1997), examining the two subtypes through their relation to boredom. Previous research had indicated that a sense of boredom was common to both covert and overt narcissism (Wink \& Donahue, 1996), however, the source of this boredom was believed to vary based upon their respective personality organization. The study authors therefore compared the scores on the WGNS (Wink \& Gough, 1990; a measure of overt narcissism) and the NPDS (Ashby, 1979; a measure of covert narcissism) with scores on the Boredom Proneness Scale (Farmer \& Sundberg, 1986), among a sample of 106 women undergraduates. As anticipated, both measures of narcissism were positively correlated with boredom proneness. However, a covert and overt distinction arose among the Boredom Proneness Scale's subscales. Specifically, only the WGNS was found to correlate with the scale tapping a sense of restlessness when confronted with external restraints on behavior, which the authors attributed to the overt narcissists sense of aggression and sense of superiority. Conversely, the NPDS was significantly correlated with subscales indicating boredom arising from a sense of purposelessness and a lack of internal stimulation (posited to reflect the sense of depletion attributed to the covert narcissist).

Similar results were found in an MMPI-2 analysis conducted by Sawrie, Watson, Sherbak, Greene, \& Arredondo (1997). Patients identified as alcoholics completed the MMPI-2, and their results were compared against a group of psychiatric controls. Researchers (e.g., Goldman \& Gelso, 1997) have theorized that ingestion of alcohol or other substances serves as a means of acquiring a false sense of self-esteem (i.e., compensating for an undeveloped grandiose self), or as a means of self-soothing following narcissistic injury (i.e., compensating for an 
undeveloped idealized parental image). It was expected that the alcoholic patients would score significantly higher than controls on measures of overt narcissism, while being comparable on measures of covert narcissism. The general assumption suggested that by ingesting alcohol and drugs, the narcissistically injured person either is acquiring the confidence and self-esteem so painfully lacking (a defect in the grandiose self) or is using the substances to calm and protect himself or herself (a defect in the omnipotent, idealized parental image).The relationship between the two forms of narcissism and alcoholism defied such simplistic categorizations, however, the distinction remained supported, as the overt measures of narcissism were found to correlate with mania and ego-inflation, while the covert measure was found to correlate with depression and anxiety.

A further development in the covert/overt distinction was made with the development of the Hypersensitive Narcissism Scale (HSNS; Hendin \& Cheek, 1997), a 10-item self-report measure of the hypersensitive form of narcissism derived from Murray's (1938) Narcissism Scale. In the development of the HSNS, only items from Murray's Narcissism Scale that demonstrated significant positive correlation with two MMPI-based measures of covert narcissism, the NPDS (Ashby, Lee, \& Duke, 1979) and the Narcissism-Hypersensitivity Scale (Serkowenek, 1975) were retained. Across three validation samples, the HSNS, the NarcissismHypersensitivity Scale, and the NPDS demonstrated significant positive correlations with each other and negative correlations with the Narcissistic Personality Inventory (Raskin \& Hall, 1979). Hendin and Cheek (1997) further compared the correlations that both the Narcissistic Personality Inventory (Raskin \& Hall, 1979) and the Hypersensitive Narcissism Scale (Hendin \& Cheek, 1997) produced with the Big Five Inventory (John, Donahue, and Kentle, 1991). Ultimately, the Hypersensitive Narcissism Scale was positively correlated with Neuroticism and 
negatively correlated with Openness and Extroversion. Conversely, the Narcissistic Personality Inventory was positively correlated with Extraversion and Openness, while being negatively correlated with Agreeableness. Hendin \& Cheek (1997) concluded that the contrasting presentation of the two types of narcissism was supported by these analyses.

Contemporary research has continued to offer empirical support for this distinction, as a wealth of studies have examined the differing clinical, interpersonal, and dispositional patterns evidenced by the two forms on narcissism. For instance, in a 2003 study conducted by Dickinson \& Pincus (2003), a comparison between "grandiose" and "vulnerable" subtypes of narcissism among a nonclinical population was made based upon self-report questionnaires and the Personality Disorder Interview-IV (PDI-IV; Widiger et al., 1995). Individuals identified as grandiose were rated as having traits associated with the narcissistic, antisocial, and histrionic personality disorders, and demonstrated elevations on scales measuring a tendency to be domineering and vindictive in interpersonal relationships. Nonetheless, such individuals denied experiencing significant interpersonal distress, and they further tended to report Secure or Dismissive attachment styles (which the authors describe as reflecting a positive selfrepresentation). Individuals identified as vulnerable were rated as possessing avoidant character traits, and endorsed experiencing domineering, vindictive, cold, and avoidant interpersonal styles. Unlike those identified as grandiose, the vulnerable individuals endorsed experiencing significant interpersonal distress, and reported Fearful and Preoccupied attachment styles (beloved to reflect a negative self-representation).

In 2005, Fossati et al. undertook an extensive statistical analysis of the Narcissistic Personality Disorder criteria among a sample of 641 outpatients. The participants were administered the Structured Clinical Interview for DSM-IV Axis II Personality Disorders and the 
Personality Questionnaire. First, confirmatory and exploratory factor analyses were conducted to determine if the NPD could be accurately characterized as representing a unitary latent trait. Both analyses indicated that the NPD criteria ultimately loaded on two correlated factors, and this two-factor structure was consistently replicated in subsequent analyses. The authors conclude that future revisions of the DSM should provide criteria for assessing the covert variant of the narcissistic PD.

In two studies utilizing a cluster analysis methodology, Lapsley \& Aalsma (2006) reviewed the notion of types of narcissism among an undergraduate population. Participants completed a measure of narcissistic dispositions (POND, Taylor, 1995), the Superiority and Goal Instability Scales (a measure of grandiosity and superiority derived from Kohut's theory of the self; Robbins \& Patton, 1985), a general measure of mental health, and a measure of separationindividuation. In both studies, cluster analysis revealed three separate clusters: covert narcissists, moderate narcissists, and overt narcissists. Both covert and overt narcissists demonstrated significant dysfunctional adjustment and psychological symptoms, but in the case of the covert narcissist, the authors remarked: "One does get the sense, here, of narcissistic grandiosity and entitlement lurking behind a façade of personal inadequacy, inferiority and vulnerability (p. 68).” The author's further note that Kohut's notion of adaptive narcissism was also supported, as the moderate narcissists were typically characterized with fewer adjustment difficulties and psychological symptoms, a finding highly similar to that of earlier research (e.g., Wink, 1992).

The notion of distress has also arisen as a distinguishing characteristic between the covert and overt forms of narcissism. Rose (2002), based on surveys of 262 undergraduate students, noted that overt narcissists reported both high self-esteem and greater life satisfaction. In contrast to this, covert narcissists reported low self-esteem and poor life satisfaction. Utilizing a 
structural equation modeling methodology, Rose investigated potential mediators to explain this disparity. The results indicated that self-esteem mediated the relationship between types of narcissism and ratings of happiness, while self-deception was not a significant mediator. As suggested by other research, overt narcissism may convey certain protective factors, as it is generally unrelated or inversely related to symptoms of distress (Sedikides et al., 2004). This stands in marked contrast to the more covert form of narcissism, which significantly correlated with feelings of anxiety, depression, and anger (Miller et al., 2010).

The negative implications of the covert form of narcissism were also identified during the development and validation of the Pathological Narcissism Inventory (Pincus et al., 2009). In a series of studies involving approximately 2,000 participants, the Pathological Narcissism Inventory was factor analyzed and the 7 resulting dimensions, organized into narcissistic grandiosity and narcissistic vulnerability types, were placed into a nomological network (i.e., the resulting dimensions' relationships with other constructs were examined). Although both forms of narcissism were found to correlate with various clinical difficulties, the vulnerable form was found to correlate more significantly with nonsuicidal self-injury and suicide attempts, as well as a significantly greater tendency to report the use of psychiatric treatment.

Other research has examined the covert/overt distinction utilizing broad elements of personality as assessed using the Five Factor Model, most commonly, the Revised NEO Personality Inventory (NEO PI-R; Costa \& McCrae, 1992). Several studies have provided evidence of distinctions between the overt and covert forms of narcissism, with the former regularly found to be positively related to Extraversion, while being negatively related to Agreeableness and Neuroticism (Paulhus \& Williams, 2002). Conversely, the covert form of narcissism has been found to be positively related to Neuroticism, while being negatively related 
to Extraversion and Agreeableness (Hendin \& Cheek, 1997; Miller et al., 2010). Yet even the similarity of an inverse relationship with Agreeableness is ultimately found to be divergent upon closer inspection. In a large-scale factor analysis of various measures of narcissism, Miller et al. (2011) presented evidence of a covert/overt distinction among narcissistic types. In the analysis, the same pattern of FFM traits between the types of narcissism was observed. Speaking to the shared inverse relationship with Agreebaleness, Miller et al. (2011) noted that the grandiose form of narcissism demonstrated a significantly greater negative relationship with the overall Agreeableness score, and it was more strongly associated with the facets of immodesty, altruism, and dishonesty.

With the impending release of the DSM-V, the importance of allowing for such diagnostic distinctions in the narcissistic pathology has become increasingly important. The concept of a unitary form of narcissism has proven tenuous, and it appears that a far more heterogeneous perspective on the disorder is required. To cement this point, Houlcroft, Bore, \& Munro (2012) conducted factor and correlational analyses using the Pathological Narcissism Inventory (PNI; Pincus et al., 2009), the Narcissism subscale of the Narcissism-AloofnessConfidence-Empathy (NACE; Munro et al., 2005), and a variety of other questionnaires assessing psychopathology and personality characteristics. The results of this recent study further confirmed the presence of two forms of narcissism, and even suggested a possible third dimension of narcissism, one characterized by antisocial tendencies. The authors concluded that a far more nuanced understanding of the Narcissistic PD is required, and that allowing for distinctions within the disorder is of critical importance for the upcoming DSM.

Summary Paragraph for Chapter II 
Kohut's theory of self psychology is rich and complex, but has not been subjected to a great deal of empirical investigation. The exception being, Kohut's ideas on the Narcissistic Personality Disorder, which have been studied extensively, and have supported Kohut's theory. Chapter II explored Kohut's ideas of a "covert" narcissist that has the fantasies of grandiosity seen the in common view of narcissism, but that also has an avoidant interpersonal style (e.g., shy and self-effacing). Kohut's theory of narcissism is derived from his theory of selfobject needs described in Chapter I, and indeed, it is the study of narcissism where self psychology began. The empirical support for the covert form of narcissism not only provides a scientific grounding for Kohut's work, but also offers a positive first step in providing evidence for his overall theory. The present investigation seeks to not only add further evidence to support the covert/overt distinction, but to also see if the selfobject needs described by Kohut were relevant to pathologies beyond that of narcissism, a conclusion that Kohut ultimately reached. 


\section{CHAPTER III}

\section{SELF PSYCHOLOGY AS NOT ENTIRELY ESOTERIC}

An initial review of the theory of self psychology can understandably lead one to view its concepts and propositions as largely arcane, a concern bolstered by the fact that its greatest empirical support is found in the research on the Narcissistic Personality Disorder, which is itself greatly understudied (Blashfield \& Intoccia, 2000).However, when carefully considered, the concepts articulated by Kohut $(1971,1977,1984)$ appear highly relevant to multiple theoretical approaches and clinical frameworks.

At the start of this investigation, it was asserted that self psychology is a theory of need. A central premise of self psychology is that all individuals stand on a continuum of having certain fundamental needs met (i.e., the fulfillment of selfobject needs), whereby those who have not received adequate support (i.e., poor selfobject responsiveness) remain vulnerable in the face life's stressors, and those who have stand as more resilient. However, regardless of where one stands on this continuum, continued support (i.e., selfobject responsiveness) is required for mental health.

In this understanding, one's self-esteem should not be conceived of as a static value that remains constant, but rather, a composite derived from the interaction between one's internal states and the supportiveness in their environment. As a logical consequence of this stance, in times of need, one should grow increasingly "narcissistic" (i.e., rely on other's for their restorative functions) as a means of coping. Interestingly, the negative consequence of avoiding such beneficial self-focus is particularly well-documented among therapists themselves (Figley, 2002).

Similarly, even severe pathology, while in the presence of needed selfobject support, 
should theoretically demonstrate improvements in functioning. An interesting consideration of this assertion may be seen in the foundational text of Dialectical Behavior Therapy (DBT; Linehan, 1993). Linehan describes the concept of apparent competence, referencing the tendency of patients with Borderline Personality Disorder to appear highly capable and highfunctioning at times. Linehan notes that "in the presence of supportive, nurturing individuals," the patient can display "emotional and behavioral competence" (p. 83). Linehan concludes by stating that "it is not clear why relationships have such an effect on these individuals," though she speculates that learning principles may account for the phenomena. In the view of self psychology, the temporary abatement of pathology and rise of competence makes sense, in that the individual is temporarily being offered an external buttress for failing internal structures.

Beyond the notion of specific types of support required for mental health, self psychology offers a potential explanation for the effectiveness of empathy as a component of psychotherapy (Elliott, Rohart, Watson, \& Greenberg, 2011).Kohut discussed therapy as a means of understanding and gathering data about a patient, a method for creating a sense of safety within the context the treatment, as well as tool for having the patient feel understood and accepted, which in turn can grow into a sense of self-acceptance (Lessem, 2005). Most central to Kohut, however, was empathy's capacity to activate the selfobject transference. Understanding the patient's needs, and in turn helping the patient to those, was one of Kohut's most influential contributions.

Based upon this understanding,Kohut advanced the controversial position that psychic defenses in treatment were not obstacles to be torn down. Kohut (1984) suggests:

"The so-called defense-resistances are neither defenses nor resistances. Rather, they constitute valuable moves to safeguard the self...The patient protects the defective self so 
that it will be ready to grow again in the future, to continue to develop from the point in time at which its development had been interrupted. And it is this recognition, deeply understood by the analyst who essentially sees the world through his patient's eyes while he analyzes him, that best prepares the soil for the developmental move forward that the stunted self of the analysand actively craves. Such recognition serves the patient better than anything else the analyst can offer." (p. 141)

In the self-psychological approach, the therapist does not set about challenging defenses, nor does the therapist conceptualize a defense as a pejorative "resistance" on the part of the client. Conversely, understanding and acceptance of the patient's internal reality, gained by empathically connecting with the client, allows the client to experience a sense of safety and confidence that makes such defenses less necessary.

The echo of this approach is seen in the contemporary usage of Motivational Interviewing, though the two theories share no explicit connection (Miller \& Rollnick, 2002). Miller \& Rollnick argue that rather than attacking resistance with "advocacy responses," the treatment is best served by trying to "understand the reason for resistance behavior and for dissonance in the counseling relationship" (p. 99). Miller \& Rollnick contend that "intrinsic motivation for change arises in an accepting, empowering atmosphere that makes it safe to explore the possibly painful present" (p. 12). The importance of empathy, understanding, and the manner of responding to resistance, are comfortably suited to a self psychological perspective.

Further, the notion of a "disordered self" that has been injured through early interpersonal experiences may be readily connected with traditional cognitive therapy. For instance, Beck (1992) speaks to the importance of core beliefs, which were believed to grow from childhood 
experiences "as the child interacts with significant others," among which a fear of "unlovability" is core (p.166). Not only are the core ideas described as "central ideas about the self," but are described in extreme cases to be "almost continuously activated."

It is with some trepidation that the preceding examples are offered, as it would be disingenuous to not acknowledge the profound differences between the self psychology approach that has been discussed and the compared theories of motivational interviewing, dialectical behavior therapy, and cognitive therapy. Nonetheless, these similarities, brief though they may be, allow for the important realization that Kohut's proposals are not entirely alien to a clinician unfamiliar with his work. Indeed, his conceptualization of psychopathology, his belief in the importance of empathy, and the potential effectiveness of selfobject experiences, all may find concepts in subsequent theories, which though not direct analogues, nonetheless appear to bear a family resemblance.

However, the single greatest congruence appears to rest within the theory of attachment, which speaks to the importance of early developmental experiences in the development of later relationship styles and pathology. Accordingly, the next chapter is dedicated to both describing the theory of attachment, as well as its relationship to Kohut's self psychology.

\section{Summary Paragraph for Chapter III}

To the unfamiliar reader, self psychology can seem largely confusing and difficult. Kohut's original works are particularly susceptible to this criticism, appearing both dense and jargon-laden. Nonetheless, his fundamental assertions are not as foreign as they first seem. Chapter III notes how several of Kohut's ideas can be found in more recent theories and treatment approaches, such as Motivational Interviewing. Although this is not to suggest a kind of equivalency, it is the present author's assertion that constructs to be measured in the present 
study (i.e., the selfobject needs of idealizing, mirroring, and twinship) are relevant to clinicians and researchers from a wide spectrum of theoretical backgrounds and perspectives. Consequently, if the hypotheses proposed are supported, results of the proposed study will help to specify why and what aspects of empathy and social support are valuable for supporting the human self in the endeavors of behavioral change and developing emotional health. 


\section{CHAPTER IV}

\section{ATTACHMENT AND SELF PSYCHOLOGY}

\section{Attachment Theory Overview}

The concept of attachment has become ubiquitous in the psychology literature, and it is widely hailed as one of the most critical developments in the study of human behavior (Rutter, Kreppner, \& Sonuga-Barke, 2009). The attachment literature is broad and developing, and in the years since its initial explication, attachment theory has proven relevant for countless domains, ranging from psychopathology to adult romantic relationships (e.g., Hrdy, 2009; Polan \& Hofer, 2008). Yet throughout its development, a central focus has remained the determination of the factors that underlie a child's sense of security with parental figures, and the results such security, or a lack thereof, has on the child's subsequent development.

The opening development in the concept of attachment was John Bowlby's (1958) initial recognition, and subsequent assertion, of infants' biologically-based, yet selective, attachment to non-interchangeable persons following a history of contingent social interactions. This revelation, though taken largely as a presupposition in contemporary thought, was revolutionary in its time. During his time as a London-based psychiatrist, Bowlby had become highly concerned with the apparent distress and ill-effects suffered by children who experienced repeated separations from their parents. Building upon a diverse scientific background, Bowlby considered this childhood pathology through the lens of evolutionary theory, primate anthropology, and ethology. In a landmark series of papers that were subsequently titled Attachment, Separation, and Loss (Bowlby, 1969, 1973, 1980), Bowlby constructed the theory that would later be named attachment. Throughout these papers the concept of attachment was described and explored, with Bowlby demonstrating a particular focus on the well-being of 
children and their ability to form attachments throughout the lifespan.

As early as 1950, Bowlby's emphasis on child-parent separations had begun to receive attention within the scientific community. As a result, he was invited by the World Health Organization to conduct interviews with a wide range of mental health practitioners and general clinicians, including psychologists, psychiatrists, and social workers across both Europe and the United States. Throughout these interviews, Bowlby was intensely interested in the effect of post-war conditions on children, with particular focus on the effects on children receiving institutional care, or children enduring extended separation from their parents.

As discovered by Bowlby through the course of these interviews, both such experiences exerted a significant influence on the children. The children who found themselves in the care of an orphanage were ultimately described by Bowlby as possessing an "affectionless character," a disposition which would later lead to difficulty in forming adult attachment relationships. As for the children who had experienced notable separations from their parents, these children often exhibited notable anxiety and distress. The key to mental health in adulthood, as suggested by Bowlby's extensive interviews, was that in the first three years of the child's life, the child was able to establish a relationship with its mother (or a single individual occupying the maternal role) characterized by warmth, intimacy, and mutual enjoyment.

The assertion that not only was the establishment of a relationship with the mother crucial for later mental health, but that the relationship had to have certain characteristics, was quite controversial. Indeed, it stood in direct contrast with prevailing thoughts and child-rearing practices at the time. Previous thoughts on the matter conceptualized maternal figures or caregivers as highly unspecific, based upon the belief that their worth to the infant was derived solely from the provision of food. As such, any adult who could fulfill this role could serve as 
caretaker. Secondly, young children (i.e., three and younger) were assumed to lack the mental capacity to hold a stable memory of a specific individual (parent or otherwise), and were believed to be therefore incapable of "missing" a parent. In sum, prior to Bowlby, specific parental activities (e.g., regular feeding), as opposed to the relationship between infant and child, were seen as the key components of successful child-rearing among infants.

In an early step in building his attachment theory (Robertson \& Bowlby, 1952), Bowlby began to directly observe a small group of children housed in residential nurseries. During the course of this observation, Bowlby noted that there were three phases of behavior that characterized the infants' behavior at the nursery, both following the parents' departure, as well as upon the parents' return. The first such phase was labeled "protest," and lasted anywhere from a few hours to several days. During this phase, the child offered protest against the parents' departure, crying and calling for their return. The child often appeared upset and frightened during this phase, displaying notable emotional distress.

If the parents were to return during the protest phase, the reunion was usually capable of soothing the child. However, if the absence grew pronounced, the child was said to enter a second phase, which Bowlby labeled as "despair." During this phase, the child's protests weakened and possibly disappeared, and the interventions of non-attachment figures (such as the nurses caring for the children) were rebuffed. If parents returned during this phase, the children often responded with frustration or ambivalence. The children were found to sometimes display sudden bouts of anger, and a fear of separation was noted as a potential outcome subsequent to these interactions.

The final phase required an even more extended parental absence. This phase, labeled as "detachment," was characterized by the child offering no visible signs of protest. In many 
senses, the child may have appeared to have adjusted well to the parent's absence, as the crying and resistance of the previous two phases were not observed. Instead, the child would demonstrate a sense of resignation, typically becoming compliant with the present nurses. When the parent would return during this phase, the child did not appear enthused by the reunion. Indeed, the parent would often be avoided or ignored, as though they were not recognized. In extreme instances, this avoidance could last for multiple days, and the child could even present rejecting or defiant behaviors.

Such investigations offered firm evidence in support of Bowlby's initial theories. Namely, there appeared to be a critical connection that the child developed with a specific person, and that connection required regular contact to maintain it. Further, continuation of this contact for the first three years of life seemed to be a key component in the course of healthy development. Conversely, disruptions in this connection appeared to place the child at risk for future psychopathology.

Perhaps even more controversially, Bowlby focused his explanation of this need for attachment with a primary caregiver through the prism of natural selection. Bowlby reasoned that species-specific behavior, much like physical developments, grew as a result of selection pressures leading to reproductive success. And indeed, other primates were found to exhibit such attachment behaviors in the first year of life, suggesting the existence of an instinctuallyguided attachment behavior system (Mason \& Mendoza, 1998). This system, according to Bowlby, must therefore increase the likelihood of survival, thereby increasing the likelihood of finding reproductive success.

Bowlby theorized that one such selection pressure was that of predation. Ground-living primates were faced with a difficulty that their tree-inhabiting counterparts were not. Namely, 
they did not have an environmental location to provide protection from predators (e.g., high up in a tree). Instead, human groups were initially forced to travel for food, living a largely nomadic existence. As such, the attachment figure was likely the only "location" which could provide a semblance of protection for the infant, as separation from the attachment figure could result in almost immediate harm or death (Hinde, 1974).

Bowlby thus argued that the need to form attachment relationships was an evolutionaryprovided mechanism to help assist in the infant's safekeeping. Because human infants are provided with this innate behavioral mechanism, they know to respond to any consistent caregiver interactions in their early years by forming a strong emotional bond with that person. Following the establishment of this bond, the child would seek to ensure close proximity with the caregiver.

Bowlby's theory was both consistent and theoretically tenable, but it would receive perhaps its greatest support from the development of the separation-reunion laboratory procedure known as the Strange Situation (Ainsworth, 1967). This procedure was developed by Mary Ainsworth as she attempted a cross-cultural replication of an observational study of Ugandan mother-child interactions. Ainsworth observed 28 infants and intended to identify and catalogue attachment behavior as it became specified to particular individuals. During this process, Ainsworth articulated what would become the two central concepts of the attachment theory: (1) use of the mother as a secure base for exploration, and (2) returning to the mother for safety. Though initially considered signs of developing attachment, these two behaviors have since grown to be recognized as the central indicators of infant security.

Unexpectedly, Ainsworth found that insecurity in the child's attachment was not simply a reflection of the parent being absent. Indeed, some of the children displayed signs of insecurity 
even in the presence of the mother. This unexpected result was later investigated in a study of 26 mother-child dyads in Baltimore. At the end of this second study, which included observation of the child-parent interactions at the family home, the participating dyads participated in the Strange Situation Procedure. During this procedure, the dyad was introduced to a friendly playroom, and while in the playroom, the mother would leave and return on two occasions, providing two instances of maternal absence and two instances of parent-child reunion. During the mother's absence, the child was left with a friendly lab assistant on one occasion, and was left alone on the other occasion. Throughout the procedure, the child's behaviors were carefully observed and examined.

Of these 23 dyads, 13 of them responded as anticipated. As expected, these 13 infants generally felt comfortable exploring the room and playing with the toys when the mother was present. When the mother left the room, the child would become distressed, and would seek proximity or contact with the mother upon her return. Following the reestablishment of the bond, the child would return to playing and exploring. These infants were described as having a secure attachment. The mothers of these children also displayed certain tendencies, which would later be correlated with their "at home" behaviors. These mothers were found to recognize their child's communications, understand them, and quickly respond in the appropriate fashion.

The remaining 10 dyads were described as insecurely attached, though there were differences among them. Four of the infants displayed a sense of distress throughout the entire procedure, regardless of their mother's presence. When the mother returned, the child would display a sense of anger, and did not become comfortable enough to return to play. Examination of the in-home behaviors, as well as responses in the Strange Situation revealed that the mothers of these children demonstrated a tendency towards insensitivity regarding their children's cues, 
ignoring them and responding inconsistently. These children would later be categorized as sinecure-ambivalent/resistant.

The six other infants (of the 10 insecurely attached) did not display outward signs of distress during the Strange Situation. Instead, they appeared to focus upon the toys in the lab regardless of the mother's presence or absence. Indeed, following the mother's return, they often ignored her. Examination of the home-records indicated that the mothers of these children were found to be rejecting of the infants' attachment behaviors. These children were labeled as insecure-avoidant.

It thus appeared that the Strange Situation had proven capable of offering insight into the relationship between the infant and the mother during the first year of life. Children who managed to establish a secure attachment had been able to utilize the mother as a secure base for exploration and play. Though the initial attempt involving this procedure had employed a small sample, Ainsworth's later replication provided similar results (Ainsworth, Bell, \& Slayton, 1971). A further consistency was the similar distribution of the attachment types across these studies, as most children were found to be securely attached (65\%), 21\% avoidant, and 14\% ambivalent.

These findings have been subjected to numerous replications, and the distinction between children with secure versus insecure attachments has been found to be robust in many studies (Carlson, Sroufe, \& Egeland, 2004; Sroufe et al., 2005; Weinfeld, Sroufe, Egeland, \& Carlson, 2008). Children demonstrating secure attachments have been found to be happier, have greater social skills, be rated as having a greater sense of self-agency, have higher self-esteem, and demonstrate a greater capacity for emotional regulation. Progressing to adolescence and then adulthood, children with secure attachments consistently demonstrated significantly less 
psychopathology and greater satisfaction in romantic relationships (Carlson, 1998).

Though the attachment styles and their importance for future functioning had received strong empirical support, these initial studies had focused upon an observable, behavioral demonstration of attachment. However, in the landmark study of Main, Kaplan, \& Cassidy (1985), the notion of an internal representation of attachment was explored. In this study, 40 sixyear-old children who had participated in the Strange Situation previously (approximately five years ago), were observed with their mothers and fathers. Mothers and fathers were independently administered the Adult Attachment Interview (George, Kaplan, \& Main, 1984), an interview designed assess for the presence of an internal working-model of attachment. This interview outlined a specific series of queries and follow-up queries regarding the parent's perception of their own attachment experiences, as well as their perceived effect of these attachment experiences on their own personalities. These interviews were ultimately transcribed and analyzed (Main \& Goldwyn, 1984).

Interestingly, the parents' style of discourse during the interview was found to reliably distinguish between their children's attachment styles. The parents' whose children had demonstrated secure attachments in the Strange Situation, were found to exhibit "coherent" and "collaborative" recollections of their own attachment experiences, regardless of whether the experience in question was perceived as positive and negative. These parents were labeled as secure-autonomous.

The parents whose children had demonstrated an insecure attachment were found to separate into two groups. One group initially offered a positive description of their own parents, later being unable to support this positive description, or perhaps even directly contradicting it. These parents were labeled as insecure-dismissing, and their children were found to demonstrate 
an insecure-avoidant attachment in the Strange Situation. The second group of parents with insecurely attached children were found to give preoccupied responses to the interview queries, often providing long and winding protocols, frequently providing confusing shifts in emotion and direction. These parents were labeled insecure-preoccupied, and their children were found to demonstrate an ambivalent attachment style in the Strange Situation.

Although these categorizations appeared both distinct and reliable, some parents did not appear to fit well within any of the available categories. These individuals often spoke of traumatic events, and sometimes appeared to demonstrate subtle disruptions in reality testing (e.g., providing highly improbable explanations of causality), and their response protocols were characterized by themes of trauma, abuse, and loss (Hesse \& Main, 2000). In 1989, a fourth classification was officially added to the Adult Attachment Interview to accommodate these parents, labeled unresolved disorganized (Hesse, 1996).

In a fashion similar to that of Adult Attachment Interview responses, the behavior of children in the Strange Situation also appeared to lack a category to adequately describe all of the participants. In Main and Solomon's 1996 study, a category for disorganized attachment was proposed to account for children whose responses during the Strange Situation did not appear readily accounted for by the secure/insecure distinction. The roots of this study dated back to Main's (1973) doctoral dissertation, wherein she became increasingly focused on six children which could not be defined within Ainsworth's original three categories. These infants, as well as infants found in other studies (e.g., Main and Solomon, 1986, 1990), demonstrated a range of unique behaviors. For example, the children might simultaneously approach the parent while averting their head, suddenly freeze, or adopt and maintain odd postures and positions. These children were labeled as displaying a disorganized/disoriented attachment. 
This fourth category was largely characterized by the experience of maltreatment, a factor strongly supported in a recent meta-analysis (Cyr, Euser, Bakermans-Kranenburg, \& van Ijzendoorn, 2010). The disorganized behavior found in the children was believed to reflect the child's inherent dilemma of viewing the parent as a source of fear, while also being instinctually guided towards the parent to relieve its distress. This was described as "fright without solution" (Maine \& Hesse, 1990). Further, the parents of such children, who themselves classified as unresolved/disorganized, often displayed anomalous behaviors towards the child, which even if not physically harmful, could be found to induce a sense of threat within the child (Hesse \& Main, 1999, 2000, 2006). This category, now commonly accepted, has been found to demonstrate significant correlations with adult pathology (Carlson, 1998; Ogawa, Sroufe, Weinfeld, Carlson, \& Egeland, 1997).

By way of summary, the attachment research identified the psychological importance of a child forming a bond with a specific individual who could serve as a source of protection. The result of forming a secure attachment with this individual was that the child could build upon this "secure base" and explore its environment. Moving through life, this secure attachment provided a number of psychological benefits. Further, this sense of attachment would crystallize into an internal representation of attachments, which would impact an adult's child-rearing practices, as well as the adult's social and romantic relationships. As one might expect, the formation of a secure attachment would generally lead to a secure internal representation of attachment relationships, thereby yielding more rewarding interpersonal relationships. However, insecure or disorganized attachment, on the part of the parent or the infant, was found to have several deleterious effects. In short, parent-child interactions would remain an important component of a person's entire life. 
Attachment and Self Psychology: A Comparison

It requires only cursory review of the theories of self psychology and attachment to recognize certain similarities between them, and indeed, both theories offered radical new ways of conceptualizing the importance of parent-child interactions in the course of development, as well as the manner in which psychopathology develops. Both Kohut and Bowlby placed the importance of human relationships at the core of their theories, and further noted that such relationships would remain crucial throughout the lifespan. Yet beyond relationships in general, both Kohut and Bowlby paid special attention to the parental relationship, offering parental responsiveness as perhaps the single most critical element of the child's psychological development, and ultimately charting the course towards healthy development versus psychopathology. Indeed, some theorists have described the selfobject needs described by Kohut as reflecting the core of the "attachment motivational system" (Lichtenberg et al., 1992, p. 136).

Both Kohut and Bowlby represented a marked departure from their psychoanalytic peers, as traditional Freudian analyses focused upon themes of intrapsychic conflict over internal drives. In traditional psychoanalysis these themes were explored through an examination of the patient's fantasies, desires, and dreams, as opposed to the "real events" of the patient's childhood. Kohut and Bowlby both offered a challenge to this perspective, instead focusing on an innate need for relatedness, and an insistence that undue focus on intrapsychic conflict rendered the analyst incapable of recognizing the pain caused by parental unresponsiveness.

Yet before progressing to an analysis of the similarities between the two theories, it is worth noting how little self-admitted overlap exists between the two. For instance, Kohut made no explicit references to Bowlby's work, and it has been speculated that this was not an accidental oversight. Mollon (2003) notes that Kohut viewed attachment theory as a form of 
psychology inherently distinct from his psychoanalytic approach, drawing a particular contrast between the methods each approach utilized. Kohut argued that the ideal manner with which to gain understanding of a patient's internal world was that of empathic immersion, and through this process the analyst could come to understand the analysand's internal structures and developmental needs. In essence, this was an attempt to gain an "inner perspective" of the patient. From Kohut's viewpoint, the seemingly external perspective of attachment theory was insufficient, and he was generally wary of incorporating developmental research into his own theory (Carr \& Cortina, 2011).

Bowlby, conversely, does offer a direct reference to Kohut in his 1988 book, A Secure Base. In it, Bowlby writes:

I believe, in adults who have developed the kind of personality...that Winnicott describes as 'false self' and Kohut (1977) 'narcissistic'...What are being excluded in these pathological conditions are the signals...that would activate their attachment behavior and that would enable them to love and to experience being loved. (p. 34-35)

This brief reference to Kohut on the part of Bowlby, and Kohut's lack of reference to Bowlby whatsoever, highlights the fundamental challenge in examining the points of convergence between the theories. Namely, we can only speculate as to the aspects of the theories which appear compatible with the other, and apparent compatibility is not to be taken as complete agreement or equivalence. Instead, in the sections that follow, each of Kohut's posited selfobject needs will be examined from the perspective of attachment theory, with an eye towards its ultimate compatibility.

Mirroring - Attachment 
Kohut described the need for mirroring as the need for acceptance and confirmation of one's sense of self. In the expression of this need, the child looks to the parents in the hopes of being recognized, admired, and praised.

Returning to the evolutionary perspective underlying Bowlby's attachment theory, a basic capacity to mirror others can be viewed as an evolutionarily-beneficial skill. Indeed, the ability to recognize the intentions and emotions of other members of the species is an important component of social interaction, as gleaning the status and intent of a member of one's own group is often crucial for survival (Pfennig \& Scherman, 1995). In the case of human beings, this ability transcends the mere recognition of another creature's emotions and potential futureactions, instead allowing us to draw parallels between our own internal states and those of others (Stern, 2006). In essence, human beings have the ability to place themselves within the mind of another.

Kohut's description of empathic immersion fits neatly within this framework. According to Kohut's understanding, empathy allows one to gain access to another person's psychological state. It is a kind of data-gathering technique that he perceived to be synonymous with vicarious introspection. Such abilities would obviously be useful in the context of a group interaction, but much more importantly for Kohut, the recognition and admiration pulled for in the mirroring selfobject needs required them. In other words, the parent cannot connect with or recognize the child's unique and special nature in order to mirror it, if the parent cannot first connect with the child's internal world. This recognition has thus led some theorist to describe the mirroring selfobject function as a uniquely human intersubjective ability (Cortina \& Liotti, 2010).

The recognition of a child's internal world was described as particularly important by Ainsworth (Ainsworth, Blehar, Waters, \& Wall, 1978), who viewed such maternal sensitivity as 
a crucial element of secure attachment. Indeed, Ainsworth description of high maternal sensitivity included being "able to see things from the baby's point of view" (p. 5), read the baby's signals, and respond to them in a way which leaves the infant satisfied. Conversely, insensitive mothers were defined as being "geared almost exclusively to her own wishes, moods, and activity" (p. 7). It requires little analysis to reveal that such "insensitive" parents would be hard pressed to offer the mirroring Kohut suggested was necessary. Thus, according to both Kohut and Ainsworth, the capacity to recognize and respond to the child's internal world is a critical parental responsibility.

\section{Idealizing - Attachment}

Kohut described the need for idealizing as the need to merge with a calm, strong and wise selfobject. In the expression of this need, the child looks to the parents as a source of inspiration, and is able to draw strength through a connection with the parent

The notion of an idealized selfobject also fits well within the attachment theory framework. Notably, Bowlby's early definition of the attachment figure was someone that was perceived as more powerful than the child. Bowlby theorized that successfully developing a secure attachment allowed the child to develop a sense of oneself as being worthy of love and care.

Returning again to the evolutionary theory that guided Bowlby, attachment is ultimately defined as a means of obtaining security for the child. The outside world is hostile toward the infant and is full of potential predators. As such, the infant can only survive by looking to the parent as a source of safety and protection. Until the child grows older and stronger, learning the skills needed to protect itself, the strength of the parent is the child's only hope.

In Kohutian terms, interactions with an idealized parent also allows the child to 
eventually gain the capacity to self-soothe and explore. The recognition of the parent as a source of strength that can be returned to in times of stress is highly similar to Bowlby's description of the benefits of secure attachment, and in both cases, these benefits are behaviorally manifested in the capacity to more confidently interact with one's environment.

Twinship - Attachment

Kohut described the need for twinship as the need to see and understand someone else, and to have that same recognition and understanding returned in kind. The expression of this need is highly variable throughout life, beginning as a primitive desire to merge with another, and culminating in an appreciation of being both separated and connected with the outside world.

In evolutionary terms, connecting with one's peers carries an obvious benefit. Social species require group interactions, as well as the ability to place oneself in the context of the group as a whole. Indeed, the twinship needs expressed by gender-specific behaviors provides a means to successfully interact with a larger social structure.

Nonetheless, such a need does not appear to be directly connected with the role of attachment. While secure attachment is indeed associated with better social interactions (Bohlin, Hagekull, \& Rydell, 2000) the twinship need does not appear to hold an immediate analogue with attachment theory.

\section{Clinical Similarities}

Both Kohut and Bowlby looked to the early experiences of life as a means of understanding later psychopathology, directing attention to specific needs that children needed to have met. Bowlby emphasized parental responsiveness to attachment needs as key, while Kohut directed attention to the needs for mirroring, idealizing, and twinship. Further, both theorists argued that an increased capacity to establish meaningful relationships with others was a critical 
indicator of successful treatment, and both detailed a broadly similar approach to treatment.

Bowlby, for instance, described that a history of unresponsive (or in more troubling cases, abusive responses) parental care could result in intense fears of separation. The work of therapy, he noted, could not truly begin until a new safe space within the context of therapy was created, and the therapist could step into the role of an attachment figure. In other words, the patient's concerns had to be validated, and the consequence of the early parental failures had to be explored.

This description is highly similar to that of Kohut, who noted that as selfobject needs occurred in the course of therapy, they had to be empathically explored and then explained to the patient. In this fashion, the therapist undertakes the responsibility initially held by the parents. Undergoing this process would ultimately allow the patient to restart a previously frozen developmental path.

One might consider the case of the disorganized attachment style as a means to explore the theories' similarities. As detailed previously, the disorganized attachment has found to correlate significantly with the experience of abuse or trauma at the hands of the parent (Main \&Solomon, 1986). This experience places the child in the impossible position of holding the parent as an object of fear and safety, simultaneously. This experience has been associated with a number of undesirable outcomes, but in Kohutian terms, has been associated with the experience of self-fragmentation (Liotti, 2004).

As conceptualized by Bowlby, during the course of therapy with such an individual, the attachment-based trauma would likely become activated, thereby bringing pathological internal working models to mind. Subsequent to this activation, the patient would struggle to form a sense of attachment with the therapist, thereby limiting the ability of the patient to "explore" 
safely within the therapy. With this obstacle in place, few therapeutic gains can be made.

The Kohutian understanding provides a ready response to this challenge. In contrast to many other psychoanalysts (e.g., Kernberg), Kohut argued for a methodical effort at understanding the patient's perspective, as opposed to identifying and challenging the patient's defenses. Through an extended process of empathic immersion, the therapist could grow to understand and appreciate the patient's needs, and this feeling of being truly understood could prove profoundly meaningful for the patient. With time, this feeling of being understood can help the patient begin to feel safe and trusted, while the therapist's explanation of the patient's needs can help to resurrect formerly dormant restorative capacities. The patient can eventually experience the therapist as a trustworthy figure that will accept them, as the therapist's empathic exploration of the patient can create the very safe space that Bowlby identified as necessary.

Narcissism and Attachment

Although there are theoretical points of convergence between the self psychology of Kohut and Bowlby's attachment theory, there has been little empirical research connecting the two theories. An important exception to this observation, however, has been found in the realm of narcissistic personalities. Research has examined the childhood experiences of narcissistic adults, suggesting that the narcissistic pathology may have it roots in attachment-disruptions. Returning to Kohut's conceptualization of the narcissistic personality, the covert form of narcissism and its corresponding attachment experiences provide a means to examine the potential convergence between the theories.

The aforementioned categories of attachment have been found to be highly stable and influential over a significant period of time (Hamilton, 2000). However, building upon the work of Ainsworth (Ainsworth \& Bell, 1970), Hazan and Shaver (1987), argued that the internal 
working models derived from ones attachment experiences could identify the likely style of one's adult romantic attachment styles. This model was later updated (Bartholomew \& Horowitz, 1991) to fit four-categories of attachment (secure, preoccupied, fearful, and dismissing), and later separated by two primary dimensions: anxiety and avoidance (Brennan, Clark, \& Shaver, 1998).

Attachment anxiety is defined as a sense of preoccupation and fear regarding the potential for abandonment and rejection, leaving the individual with a sense of a threat hanging overhead. This anxiety is theorized to arise from inaccessible or unresponsive parenting, leading to a negative model of the self (Bowlby, 1973). This sense of possible rejection appears highly applicable to the covert form of narcissism, as the covert form of narcissism is more immediately connected with negative emotions and the sense that one might be overwhelmed by external difficulties (Mikulincer \& Florian, 1998). The projected sense of disapproval and the avoidant presentation of the covert narcissist usually communicates a sense of anxiety, a fact which may reflect experiences with an unreliable attachment figure (Smolewska \& Dion, 2005).

Attachment avoidance is defined as an avoidance of relationships, excessive emphasis on self-reliance, and a sense of discomfort with intimacy and closeness. This avoidance is theorized to arise from rejecting or unemotional caregivers (Brennan et al., 1998). As with the attachment anxiety, such avoidance appears congruent to the hypersensitivity of the covert narcissist. The covert narcissist is seen to avoid being under the observation of others, and a fundamental sense of insecurity prevents them from comfortable engaging with others in a personal way.

The dimensions of anxiety and avoidance can be further examined so that their respective elevations and declinations can offer a description of the individual's attachment style. For example, elevations of both anxiety and avoidance is described as a fearful attachment, being 
high on avoidance and low on anxiety is described as a dismissive attachment, and those high on anxiety and low on avoidance are described as having a preoccupied attachment (Miller et. al., 2011).

\section{Empirical Research on Narcissism and Parental Behaviors}

Evidence regarding a connection between parenting style and narcissism has received a modest amount of support in the literature, indicating that narcissism may indeed be more likely to arise based upon early developmental experience (a conclusion that both Kohut and Bowlby would anticipate). For instance, in a study of adolescents, scores on the Narcissistic Personality Inventory were found to positively correlate with "negative" parenting styles, with negativity describing qualities such as infrequent supervision, use of corporal punishment, and inconsistency in enforcing rules (Barry, Frick, Adler, \& Grafemen, 2007).Similarly, in a study of college-students and adolescents (Horton, Bleau, \& Drwecki, 2006), elevations on the Narcissistic Personality Inventory were associated with parental warmth, while the parental tendency to monitor the child's behavior and whereabouts was negatively correlated with scores on the Narcissistic Personality Inventory. Finally, in a group of adults (ranging in age from 18 to 52), scores on Narcissistic Personality Inventory were positively correlated with retrospective ratings of parental coldness and over-evaluation (Otway \& Vignoles, 2006).

Studies utilizing the O’Brien Multiphasic Narcissism Inventory (OMNI; O’Brien, 1987)

offered further evidence of the importance of parental behaviors in the development of narcissism. In a study involving the self-report responses of 335 undergraduate students (O'Brien 1987), maladaptive narcissism as measured by the OMNI was positively correlated with recollections of Authoritarian parenting styles, while in a separate study involving 370 undergraduates (O'Brien 1988), the OMNI demonstrated no meaningful correlation with 
Authoritativeness.

A longitudinal study of narcissism conducted by Cramer (2010) provided strong evidence for the importance of parental behaviors on the development of narcissism. Participants were assessed at ages 3 and 23, gaining information about maladaptive narcissism, defense mechanisms, and parental behavior. Cramer concluded that "responsiveness that is inappropriate to the child's developmental level" (p. 26) had a direct effect on the development of narcissism, but this development depended upon the child's proclivity towards narcissism, a finding supported by other research (e.g., Barry et al., 2007).

\section{Narcissism and Adult Attachment}

Moving towards adult attachment specifically, several studies have looked at the connection between narcissism and adult attachment. Fortunately, this research has also considered the cover/overt distinction within the narcissistic personalities, allowing for an examination of the differing pattern of relationships between them with respect to attachment.

In 2003, Dickinson \& Pincus (2003) conducted an analysis of the covert and overt distinction in narcissism through an examination of personality disorder criteria, interpersonal difficulties, and adult attachment styles. The general findings of the study supported the covert and overt distinction in narcissism, but also provided important evidence for the distinction of the two constructs in relation to attachment. The narcissistic individuals identified as overt (labeled as grandiose), typically demonstrated adult attachment styles reflecting positive selfrepresentation (i.e., Secure and Dismissive). Conversely, the covert narcissists (labeled as vulnerable), reported adult attachment styles that are reflective of negative self-representations (Fearful, Preoccupied).

A 2005 study conducted by Smolewska and Dion (2005), examined the responses of 171 
female respondents on measures of overt narcissism, covert narcissism, and attachment. A canonical correlation analysis revealed covert narcissism demonstrated a strong connection with both anxiety and avoidance attachment, though it was more strongly associated with anxiety attachment than with avoidance attachment.

In a comparison of four structural equation models, Otway and Vignoles (2006), 127 participants completed a range of questionnaires, including measures of narcissism and attachment. In their research, Otway and Vignoles revealed that both forms of narcissism were predicted by recollections of both parental coldness and excessive admiration. Speaking to attachment more specifically, the covert form of narcissism was predicted by attachment anxiety. No such predictor of overt narcissism was found.

In a study producing similar results as Smolewska and Dion (2005), Miller et al., (2010) examined the differing interrelations between factor 2 psychopathy, covert narcissism, and borderline personality disorder. In a study of 361 undergraduates, covert narcissism demonstrated a strong connection with both anxiety and avoidance attachment, though it was more strongly associated with anxiety attachment than with avoidance attachment. Conversely, the overt form of narcissism appeared to enjoy a secure form of attachment. Summary Paragraph for Chapter IV

Chapter IV provided a description of attachment theory, explored its development and relevant research. The theory of attachment speaks to the importance of parent-child interactions for the short and long-term mental health. Attachment theory states that the parentchild relationship can affect the child's view of self, the world, and future. This assertion is compatible with that of Kohut's self psychology, which also speaks to the importance of early developmental interactions between parent and child. Attachment research enjoys strong 
empirical support, and its theoretical congruence with Kohut's theory suggests that the operationalization of self psychology may have much to contribute to the further elaboration of attachment theory and vice versa. On the other hand, attachment theory may provide a more parsimonious explanation of phenomena of interest to self psychology, as it may render Kohut's developmental theories unnecessary. The proposed research will examine the relations between the two theories, examining whether or not they are complementary, redundant, or perhaps divergent. 


\section{CHAPTER V}

\section{SHAME}

\section{Kohut and Self Psychology's View of Shame}

Kohut began his consideration of shame early in his work, addressing the matter in the Analysis of Self (1971). Kohut described the experience of shame as arising from a disturbed narcissistic equilibrium, and indeed, the new conceptualization of narcissism offered by Kohut almost necessarily required a meaningful reconsideration of the theory of shame. In contrast to Kohut, Freudian analysis of the time had largely focused upon the emotions of guilt and anxiety, arguing that these emotions arose from frustrated sexual and aggressive desires, and paying sparse attention to the form of shame described by Kohut.

The focus on shame was in many ways a natural progression for Kohut, as his understanding of normal development, as well as his conceptualization of the development of psychopathology, was grounded in the individual's sense of self. As such, the experience of shame, which involves a negative evaluation of one's sense of self, becomes immediately appropriate. As a brief aside, the distinction between guilt and shame in this regard merits attention.

Shame and guilt share a number of common features. Most notably, shame and guilt are both self-conscious emotions that reflect a negative affect that serves to modify one's behavior (Tangney, 1995). In other words, individuals are aware of when they experience either emotion, both emotions are painful to experience, and both emotions can serve to regulate behavior. The emotions are separated, however, based upon their respective focus. In the case of guilt, the negative affect is focused upon a specific action or failure. For instance, one might experience a sense of guilt after saying something unduly harsh. The guilt then becomes focused upon a sense 
that the action in particular was uncalled for and regrettable. In the case of shame, one's entire sense of self becomes the focus of the negative affect. The experience of feeling weak, unworthy, or fundamentally flawed is a sign of one experiencing a sense of shame. In sum, guilt refers to a sense of regret over what one has done, while shame refers to a sense of regret over who one is (Cooper, 1998).

The sense of shame, given its relevance for one's sense of self, was thus a matter of some importance for Kohut. Kohut theorized that the sense of shame first arose when the child displayed its need for approval and attention from the parents, and the parents responded with condemnation. Kohut described this experience as particularly damaging to the child, not only because the experience of parental dissatisfaction is painful in and of itself, but also because in that moment the child was reaching out for praise and acceptance. Shame them resulted from the child experiencing of rejection at the precise moment when it is seeking comfort and support.

The child takes from this painful experience the lesson that its expansive and exhibitionistic fantasies are shameful and base, and must be held at bay. The normal course of narcissistic development is thereby disrupted, and the experience of any sort of grandiosity in the future will likely be experienced as threatening, as it brings with it the sense of shame that has been withheld by repressing the grandiose fantasies.

As one might expect, Kohut describes this sense of shame as therefore arising from the incongruent responses of important selfobjects (most likely, the parents). Failure to experience the needed mirroring from another, or an inability to connect with an idealized other, or even an inability to connect with another person as fellow human being (i.e., twinship), leaves the child feeling vulnerable, and the child may respond by retreating from the outside world, becoming more introverted and withdrawn (Morrison, 1994). 
The onus for this retreat is ultimately the child's perception of its selfobject matrix (i.e., the significant persons in its environment) as lacking and estranged. The child further begins to wonder if there is something within it that is lacking, some internal flaw that might explain why its parents appear dissatisfied and distant. "What is it about me that causes my parents to react to me in this way?" The child's withdrawn nature is therefore accompanied by feelings of worthlessness, inability, weakness, and isolation, the very sort of feelings emblematic of shame.

In the case of normal development, the child is able to construct an image of its ideal self, and work towards obtaining that ideal. Of course, this ideal cannot be met, and it is in the failure to meet the perceived ideal that the child begins to inadvertently develop criteria for the experience of shame (Morrison, 1994). If the child receives adequate selfobject responses, these criteria become flexible and realistic. The child's efforts are supported and celebrated, and the child is therefore able to create sense of an obtainable ideal (i.e., the child does not have an unrealistic sense of what living up to its ideals will require). Further, the child's own reasonable expectations, and the encouragement and support of the parents, allows the child repeatedly attempt to meet these ideals, thereby giving the child a sense of agency and strength.

However, when the child does not have its selfobject needs met, the idealized image takes on a rigid and unrealistic character. If this idealized sense of self could be matched, the child fantasizes, the desired selfobject responses might occur. In other words, the child hopes that by meeting this unrealistic standard, the parents will provide the sense of connection that the child desires. However, as the child continues to fail to live up to this standard, and as the desired parental responses remain withheld, the child is repeatedly forced to view itself as a failure, and the discrepancy between the child's idealized self (who the child believes would be accepted and praised), and the child's actual self, gives rise to a sense of shame. 


\section{Narcissism and Shame}

As is the case with much of Kohut's theory, narcissism provides one of the few grounds for a firm investigation of the experience of shame. Several theorists have argued for the centrality of shame in the experience of narcissism (e.g., Morrison, 1986; Broucek, 1991), suggesting that shame arises in response to a repeated failure to match one's grandiose ideals. Further, the presentation of narcissistic symptoms has been described as a defensive reaction against shame, as the narcissistic individual aggressively holds onto a sense of success so that thoughts of failure are pushed aside (Morrison, 1989). Similarly, other theorists describe narcissistic grandiosity as a means to undue one's sense of failure by demanding the admiration of those around them (Reich, 1986). In essence, narcissism as explained by the DSM is treated as something of a defensive shell to protect a vulnerable core (Miller, 1981).

As one might expect, the covert/overt distinction in narcissism has proved highly relevant the experience of shame, with the covert form of narcissism (more aligned with Kohut's description of narcissism) being theoretically and empirically linked with the experience of shame. Indeed, Glen Gabbard, a preeminent psychoanalytic theorist, argued that the sense of shame is crucial to understanding the psychology of the covert narcissist (Gabbard, 1983). It need be remembered that the covert narcissist experiences a sense of grandiosity, but the desire to connect with this grandiosity has become a source of shame and humiliation (based upon the developmental experiences described above). The covert narcissist thus fears having their grandiose nature exposed, as they regard their exhibitionistic desires as fundamentally flawed, and this negative self-evaluation gives rise to a sense of shame. "They projectively attribute their own disapproval of their grandiose fantasies onto others" (Gabbard, 1983, p. 468), and thus carefully avoid having their shameful secret revealed. 
Research has supported this differential experience of shame among the covert and overt forms of narcissism. For example, in the formerly discussed study of Hibbard (1992), 701 college students completed eight scales measuring narcissism, object relations, masochism, social desirability, and shame. The covert/overt distinction arose, with the two styles being separated based upon the experience of shame. Shame correlated negatively with the grandiose style, and correlated positively with the vulnerable style. Further, this relationship was not mediated by social desirability. Broucek's (1991) research was congruent with that of Hibbard, finding that covert narcissism was characterized by a kind of vulnerability to shame.

That the overt narcissist presents as shameless in this regard may reflect a defensive tendency. According to Pulver (1986), the overt narcissist's grandiosity is a conscious effort to suppress their sense of shame. As such, self-report measures of shame may encounter a particular defensiveness from the overt narcissist. Indeed, requests to endorse shame represent an effort to concede a painful, and actively denied, emotion. As such, the accuracy of selfreported shame can be somewhat tenuous (Scheff, 2003).

It is of some interest to note that Kohut's explanation of the experience of shame provides obvious correlates with attachment theory. According to Kohut, the development of shame ultimately arises from interactions with attachment figures who are not attuned to the child's needs. The unresponsive nature of these interactions teaches the child that the parents are unwilling to fulfill its needs, and the child's explanation of the parental reticence is that of a sense of a personal shortcoming, which in turn gives rise to shame. In essence, Kohut indirectly asserts, the emotional availability and responsiveness of an attachment figure serves as a buffer against the growth of shame.

Kohut's explanation of shame appears highly congruent with attachment theory, with 
some researchers going so far as to describe shame as an "attachment emotion" (Lewis, 1980). Accordingly, other researchers have, in agreement with Kohut, described the failures of early attachment as the primary source of shame (Kaufman, 1989). Beebe and Lachman (1988), for instance, described shame as arising from caregiver failures, arguing that these failures teach the child to not expect the caregiver to participate the in their emotion-regulation. Speaking summarily, Lewis (1992) asserted that the failures of attachment figures to provide the child with needed psychological support creates a shameful disposition. In other words, shame and insecure attachment are inexorably linked (Nathanson, 1992; Seidler, 1997).

Empirical research has supported these notions, as preoccupied attachment is associated with significant experience of shame (Bartholomew \& Horowitz, 1991). Interestingly, the dismissive attachment style has a similar defensive style to that of the overt narcissist, tending to display a defensive stance and an unwillingness to endorse negative emotions such as shame (Cassidy, 1994; Magai et. al., 2000). In sum, the attachment perspective of shame is largely similar to that of Kohut, suggesting that lack of caregiver attunement leads the child to feel unsupported by the parents, leading to a sense of shame as the child struggles to craft an explanation for the experienced parental coldness. Shame then, arises from, and contributes to, insecure attachment (Nathanson, 1992; Seidler, 1997).

Summary Paragraph for Chapter V

Kohut described much of psychopathology as arising from unmet selfobject needs (described in Chapter I), theorizing that the result of having these needs go unmet was a symptom presentation of emotional dysregulation including depression and rage. An emotion of particular interest for Kohut was that of shame. Shame, as opposed to guilt, is the experience of oneself as fundamentally unacceptable, flawed and lacking, a condition of obvious importance to 
a psychology of the self. Accordingly, a measure of selfobject needs such as proposed by the present study, is hypothesized to be highly correlated with experiencing shame. Chapter V, offered a description of Kohut's understanding of shame, as well as the empirical literature regarding shame and narcissism. 


\section{CHAPTER VI}

\section{EXISTING SELF PSYCHOLOGY MEASURES}

\section{Superiority and Goal Instability Scales}

The Superiority and Goal Instability Scale were developed by Robbins \& Patton (1985), based upon Kohut's $(1971,1977)$ psychoanalytic theory. These scales were designed to measure Kohut's proposed idealizing and grandiosity poles of the self, and were connected with future career ambitions. The Superiority Scale focused upon a sense of one's own sense of grandiosity, including items such as "My friends follow my lead," while the Goal Instability Scale reflected a lack of direction in one's life (theorized to result from disruptions in one's view of the idealized parental image), and included items such as "It's easier for me to start than to finish projects." The scale was initially developed on 453 undergraduate students. A factor analysis revealed the presence of two factors, labeled Superiority and Goal Instability. These scales were then entered as predictors in a hierarchical regression models to predict the ability of a new sample of 88 undergraduates to formulate and pursue career objectives. Both scales proved to be significant predictors.

The Goal Instability Scale has demonstrated strong empirical support, and has been found to relate to adjustment to college life (Robbins, Lesse, \& Herrick, 1993), academic performance (Scott \& Robbins, 1985), and career development (Robbins, 1987). The Goal Instability Scale has also demonstrated high reliability and identical factor structure among older adults as well (Robbins, Payne, \& Chartrand, 1990). Speaking summarily, the Goal Instability Scale demonstrates strong psychometric properties as well as theoretically-expected relationships with other variables of interest. 
The Superiority Scale has received less attention in the literature, and the research that has been conducted using the measure has produced inconsistent results. For example, in its initial development study (Robbins \& Patton, 1985), the Superiority Scale was found to nonsignifciantly correlate with self-esteem. Robbins \& Schwitzer (1988) found a negative correlation between the Superiority Scale and social adjustment, a surprising and troubling finding. It may thus be concluded that the scale's validity is mixed at best (Robbins \& Schwitzer, 1988).

Social Connectedness and the Social Assurance scales

The Social Connectedness and Social Assurance Scales were developed by Lee \& Robbins (1995). The scales were developed and validated on a sample of 696 undergraduate students. Half of the sample was used as a split-sample in order to validate the structure of the measures identified by the other half of the sample. The measure was based on Kohut's (1984) proposed selfobject need for connectedness, described by Kohut as an alter ego (i.e., twinship) need. Factor analyses ultimately identified a two-factor solution to the measure, defined as Social Connectedness and Social Assurance. The two measures demonstrated high internal reliability, high test re-test reliability over a two-week period, and a confirmatory factor analysis (done with the second sample) supported the initial factor solution. Social Connectedness was found to reflect one's opinion of self in relation to other people, while social assurance appears to reflect one's reliance on other people. Both scales are meant to reflect the larger construct of belongingness.

The Social Connectedness Scale was validated in a series of studies, and was further found to be distinct from related measures of social identity, loneliness, and amount of social support, while demonstrating expected relationships with self-esteem and anxiety (Lee 
\&Robbins, 1995, 1998, 2000).

Despite this empirical support, the scale was revised in 2001 (Lee, Draper, \& Lee, 2001), to address potential negative response bias (the original items were all written in a negative direction), alter some of the item wording, and to broaden its scope to better account for the full range of social connectedness). In the same study, Lee et al. (2001), offered support for the revised Social Connectedness Scale's validity. In its present form, the scale includes items such as "I feel distant from people." This scale has also shown potential as a mediator between social support and depression and self-esteem (Williams \& Galliher, 2006).

Aside from its initial development, the Social Assurance scale has received scant attention, becoming largely abandoned in favor of the Social Connectedness Scale. Although the two scales are believed to assess different aspects of belongingess, and demonstrated only a moderate correlation with each other $(r=.22$; Lee \& Robbins, 1995), the overlap between them may account for the Social Assurance scale's relative absence from the literature.

\section{Selfobject Needs Inventory}

The Selfobject Needs Inventory (SONI; Banai, Mikulincer, \& Shaver, 2005) represents the single most relevant attempt at deriving an empirical measure of Kohut's selfobject needs. The SONI was intended as a measure of the selfobject needs of idealizing, mirroring, and twinship, with Banai et al., 2005 further conceptualizing each of these dimensions as having an approach pole and an avoidance pole. As such, a six-factor measure was anticipated (i.e., three dimension, each with two factors). The SONI consists of 38 items, each scored according to a Likert-type scale ranging from $1=$ Not at All to $7=$ Very Much. The measure was created and developed in Hebrew, and was tested among Israeli undergraduate students.

In its initial validation study, 372 Israeli undergraduate students completed the SONI, and 
factor analyses revealed a five factor structure consisting of the following factors: hunger for twinship, avoidance of idealizing and twinship, hunger for idealization, hunger for mirroring, and avoidance of mirroring. Cronbach's alphas for the five scales were acceptable, ranging from .79 to .91 .

During its initial study, the SONI's validity was explored by examining its relationship with the aforementioned measures of Kohutian concepts, and the SONI's validity was strongly supported. For instance, the Superiority Scale was significantly related to Approach Mirroring $(r$ $=.35, p<.01)$ and Avoidance of Mirroring $(r-.34, p<.01)$; the Goal Instability Scale was significantly associated with Idealizing Approach $(\mathrm{r}=.32, p<.01)$; and the Lack of Connectedness Scale was significantly associated with Twinship Approach $(r=29, p<.01)$ and Avoidance of Twinship/Idealization, $(r=.36, p<.01)$ (Banai et al., 2005).

In a series of follow-up analyses, the SONI's relationships with a number of other constructs were considered. Speaking summarily, a canonical correlation between the set of five SONI scales and attachment anxiety, attachment avoidance, rejection sensitivity, and fear of intimacy was found to be significant: $r c=.48, F(20,290)=6.80, p=.01$. In this analysis, the hunger aspects of the SONI were significantly correlated with attachment anxiety and rejection sensitivity, while avoidance of selfobject needs were associated with attachment avoidance and fear of intimacy. A further analysis found that canonical correlation between the five SONI scales and Narcissistic Personality Inventory scores yielded a significant association between selfobject needs and narcissistic personality, $r c=.41, F(20,336)=4.30, p<.01$. Finally, A canonical correlation between the set of five SONI scales and the set of self-esteem also yielded a significant multivariate association, $r c=.36, F(25,448)=5.04, p<.01$, with hunger for mirroring and hunger for twinship significantly associated with low levels of self-esteem. 
Despite the initial study covering the development and validation of the SONI (encompassed in a single publication), subsequent research conducted by researchers beyond the measure's creators has not been supportive of the measure's validity. Further, even in its initial development problems were noted, as the collapsing of avoidance of idealizing and twinship into a single category was a theoretically unexpected result.

Review of the modest amount of research conducted with the SONI has revealed several shortcomings. For instance, Bastanfar (2009), utilizing a sample of 199 American undergraduate students, attempted to reproduce the five-factor structure identified by Banai et al. (2005). However, the five-factor demonstrated consistently poor fit, as indicated by the chi-square test of goodness of fit test, the chi-square to degrees of freedom ratio, the comparative fit index, the Tucker-Lewis Index, and the root mean square residual. Following these results, Bastanfar (2009), conducted an exploratory factor analysis, and found that after varimax rotation, a 10factor solution was identified.

Similarly, Gruber (2009) administered the SONI to 206 American undergraduates. The factor structure proposed by Banai et al. (2005), was reviewed, but the scales demonstrated highly variable internal inconsistencies, with Hunger for Idealization and Avoidance of Mirroring demonstrating unacceptable internal consistency (alphas of .69 and .60, respectively). A new factor analysis was conducted, requiring the omission of seven items due to significant cross-loadings, and ultimately concluding in a four-factor solution.

Finally, in a study of 268 American undergraduates, Canepa (2011) examined the SONI's five reported scales and their relationship to several constructs. Although Canepa did not examine the factor structure of the SONI, two important differences between her findings and that of Banai et al. (2005) were noted. First, significant inter-correlations between SONI hunger 
and avoidance scales were observed, suggesting less separation between the selfobject needs than found by Banai et al. Further, in Canepa's sample, anxious attachment was also found to correlate significantly with the avoidance of idealization/twinship, a finding in contrast to that of Banai et al.

The failure of the SONI to produce six factors in its initial validation, as well as the empirical investigations calling into question its proposed factor structure, are significant shortcomings. The source of these shortcomings is unclear, though these differences might be in part attributable to differences in culture or language, as the measure was not developed in English or among an American population. As such, though it is an intriguing attempt at tapping Kohut's proposed selfobject needs, it cannot claim to be successful in the regard.

\section{Shortcomings of the Available Self Psychology Measures}

The development of the aforementioned measures represents an important beginning for the research into self-psychological constructs. At a minimum, they offer evidence that the seemingly abstract constructs described by Kohut (1971) can be tapped, at least in part, by selfreport measures. Further, they provide preliminary evidence that self psychology principles exist in a wider context than that of the narcissistic personalities.

However, in the case of Superiority and Goal Instability Scales, as well as the Social Connectedness Scale-Revised, the measures are not designed as assessments of selfobject needs as such, though they are undoubtedly related. Additionally, these scales were not designed as measures with clinical aims in mind, though they certainly carry therapeutic implications. In essence, these measures provide empirical demonstrations of Kohutian concepts, but cannot accurately be described as measures of selfobject needs, which represent the core of selfpsychological theory. The present measure will offer an important improvement upon these 
measures by not only drawing upon the selfobject needs specifically, but also by developing a tool which is clinical in nature.

The SONI, conversely, is an effort at producing an inventory very similar to that of the present measure. Nonetheless, the SONI has not fared well in several analyses, suggesting that a more capable instrument could provide an important improvement to the literature. It is also the present author's contention that the construction of a measure based upon the presumption of an approach/avoidance axis for each selfobject need, though understandable, is not ideal. Should the theory of the importance of selfobject needs prove correct, then the absence of such needs is presumed to result in a wide range of pathologies, of which, the active avoidance of the needs is but one. Therefore, the theory upon which the SONI is developed, articulates the selfobject needs as universal, while offering the avoidance of the needs as a potential defense mechanism. Given the proposed universality of the former, and the more limited applicability of the latter, it is perhaps unsurprising that the author's of the SONI were not able to validate their suggested six-factor structure. Of course, one might also contend that a six-factor structure is simply a psychometrically burdensome hypothesis. In either case, the present measure will intend to contrast itself with the SONI by focusing upon the three selfobject needs, and by further producing a more psychometrically sound instrument.

Summary Paragraph for Chapter VI

Although Kohut's theoretical ideas appear somewhat abstract, there have been several efforts to create measures of the constructs he described. Chapter VI described these measures and their corresponding literature, as well their shortcomings that the proposed measure will address. Of particular interest is the SONI, which is very similar to the measure that the proposed study seeks to develop in a fashion that will obtain stronger validity. The SONI will be 
included in the present research as a way to establish the newly-created measure's convergent and discriminant validity. Further, the proposed measure will be directly compared against the SONI, both in terms of its predictive ability and its psychometric soundness. 


\section{CHAPTER VII}

\section{PROBLEM STATEMENT}

The theory of self psychology as advanced by Heinz Kohut (1971, 1977, 1984) emphasized the importance of early developmental experiences, most centrally, the empathic attunement of caregivers as key nutrients for fostering the capacity for healthy self-regulation and interpersonal relations. Kohut elaborated three critical selfobject needs (i.e., idealizing, mirroring, and twinship) that were ideally satiated by these early experiences, deeming the fulfillment of these needs as essential for developing a healthy sense of self, capable of sustaining ambitions, self-soothing, and caring for others and oneself. In the case of psychopathology, these needs were theorized to have gone unmet, leaving the individual with deficits in their ability to regulate self-esteem, and thereby rendering them vulnerable to mood and behavioral dysregulation, as well as difficulty sustaining satisfying intimate relations, especially in the face of life's stressors.

Although Kohut's theory has been embraced as quite useful by many mental health professionals, particularly those with a psychodynamic affiliation, there has been relatively scant empirical research examining its underlying hypotheses. It is the present author's assertion that a primary reason for the scarcity of empirical attention devoted to Kohut's theory has been due to challenges operationalizing his concepts, which has lead to a lack of adequate measures of his key constructs, especially his idea of the selfobject needs. For instance, studies using the Selfobject Needs Inventory (SONI; Banai, Shaver, \& Mikulincer, 2005), the most recent attempt to operationalize Kohut's theorized needs, has demonstrated inadequate reliability and validity across multiple studies (e.g., Bastanfar, 2009). 
To address these measurement shortcomings, the present dissertation had several objectives:

The first and primary objective was to develop a self-report measure capable of assessing the selfobject needs proposed by Kohut. The proposed strategy was designed to yield a measure that could reliably and validly assess individual differences in the selfobject needs of idealizing, mirroring, and twinship. Consequently, a 3-factor model was expected. An initial version of this measure was to be created and assessed with an exploratory factor analysis.

The second objective was to confirm the factor structure identified during the measure's initial development, via a confirmatory factor analysis. This step also provided an opportunity to compare the present measure's factor structure with that of the SONI, as well as their respective relationships with the aforementioned constructs.

The third objective was be to place this newly developed measure in a broader nomological network of theoretically related constructs pertaining to the development of self and the ability to remain resilient and socially connected when facing challenges or threats. Specifically, its relationship with measures of adult attachment, overt narcissism, covert narcissism, shame, self-esteem, idealization, grandiosity, social connectedness, and the SONI was considered. It was hypothesized that the new measure would demonstrate strong positive correlations with shame, covert narcissism, and the corresponding factors of the SONI. Small positive correlations between the new measure with overt narcissism and insecure attachment were also hypothesized. Finally, the new measure's factors of Idealizing, Mirroring, and Twinship were hypothesized to demonstrate uniquely large positive correlations with the external measures of idealization, grandiosity, and social connectedness, respectively. 


\section{Specific Hypotheses}

The present research thus investigated two core hypotheses. These hypotheses are outlined below.

A psychometrically sound measure of selfobject needs, named the AESOP (Arble Estimate of SelfObject Pursuits) can be created. This measure is expected to reliably produce a three-factor structure, with each factor corresponding to one of the following selfobject needs: idealizing, mirroring, or twinship.

The measure's validity should be demonstrated through an investigation of its interrelations correlations with other measures in a broader nomological network. 


\section{CHAPTER VIII}

\section{METHOD}

$\underline{\text { Study } 1}$

\section{Item Generation}

The initial task in creating a measure designed to assess the selfobject needs of idealizing, mirroring, and twinship was to create an item pool capable of covering the elements of these domains. As such, an extensive review of the self psychology literature, published case studies, and clinical reviews of self psychological theory was undertaken to create a list of potential items. This initial series of items was then be reviewed for clarity, brevity, lack of redundancy, precision (i.e., focusing solely upon the desired construct), and appropriate reading level.

This edited form of items was then distributed to a "rating team" consisting of two members of the present dissertation committee with interest and expertise in self psychology, as well as three clinical psychologist members of the New York Institute for Psychoanalytic Self Psychology. These additional raters were solicited via email for their willingness to review and rate potential items. Five potential raters were solicited in this way, and three agreed to participate. External members were identified through postings on the Institute's website.

A total of 51 items for Idealizing, 43 items for Mirroring, and 42 items for Twinship were created. Members of the rating team rated each item on a five-point scale with the following anchors: 1 = Poor; 3 = Acceptable $;=$ Excellent. Participating raters applied these anchors according to the item's performance across two domains: clairty and relevance. Clarity refers to the item's ability to clearly convey its meaning to the reader. In simpler terms, it assesses if the item is easily understood. Relevance refers to the item's perceived ability to reflect the construct it is designed to measure. For example, an item designed to tap a need for mirroring would be 
reviewed for its perceived ability to actually assess the need for mirroring, as opposed to another construct or selfobject need. Raters were instructed to rate the item based upon its clarity and relevance, with items requiring a mean rating of three or higher across both domains. Items with mean scores lower than 3 were discarded.

The resulting pool of items was intended to yield sufficient potential items for each of the three proposed factors. The resulting initial scale was organized into a single measure where respondents rate how descriptively the item describes them according to a seven-point scale with the following anchors: $1=$ Not at All Descriptive; $4=$ Somewhat Descriptive; $7=$ Very Descriptive.

Participants

The present study utilized a convenience sample of undergraduate students at a local Midwestern university. Participants were offered the incentive of extra credit in their courses in exchange for participation. A posting for the study was placed within the aforementioned university's online study system. This posting offered a description of the study's purpose, length of time to participate, and amount of extra credit available.

Though recruitment efforts were based on obtaining the greatest number of participants, statistical requirements were also considered. In performing an exploratory factor analysis, the sample size required is in large part a reflection of the data itself and the strength of the measure (MacCallum, Widaman, Zhang, \& Hong, 1999). As such, normally distributed data involving a measure with few item cross-loadings and high internal consistency requires a smaller sample. Nonetheless, larger samples are ideal (Costello \& Osborne, 2005). Some researchers have suggested a general ratio of 10-to-one between respondents and number of items on a measure, with others positing a minimum of 100 respondents (Mundfrom, Shaw, \& Tian, 2005). In the 
case of the present research, a more practical ratio of five-to-one was established as a prerequisite. However, the final instrument of the present measure consisted of 70 items, and 738 individuals completed the measures. Thus, the 10-to-one ratio was ultimately obtained.

\section{Procedure}

Participants were informed that in exchange for completing a measure of selfperceptions, they would be eligible for extra credit. Participants reviewed and indicated their willingness to participate by checking the consent option of an electronic consent form. Subsequent to this, participants were able to access the provided survey. The survey instructions informed the participants to "Read each item and rate how accurately it describes you, using the provided scale."

Participants completed the survey online using the university's secure research portal. Access to the survey required a unique student ID, which allowed participants to access their extra credit, while also preventing participants from completing the survey on multiple occasions. Because the survey was hosted online, participants could complete the measure at the time and location of their choice. The survey was hosted as a "forced choice" option, meaning that respondents had to provide responses to every item, and could not skip items.

Additionally, the survey would not allow participants to access the survey if they listed their age as being under 18 , or if they had not selected a box indicating that they had reviewed the provided consent materials. As the participants were informed, they could discontinue participation at any time by simply closing their Internet browser.

Analyses

A maximum likelihood exploratory factor analysis was used to determine the new measure's underlying factor structure. Items were reviewed for removal according to three 
criteria: 1) Significant loadings on multiple factors or failure to significantly load on a single factor (with factor loadings of .32 and above being regarded as significant, as indicated by Tabachnick \& Fidell, 2001); 2) Low item-total correlations with other items on the same factor (i.e., lower than .4); 3) The item's removal would increase the Cronbach's alpha value above a standard cutoff such as .70 (de Vaus, 2002). The factor structure following the removal of these items was then reviewed.

Determining the number of factors to retain in an exploratory factor analysis is a matter of some controversy, though a frequently employed strategy is to retain factors with eigen values greater than 1.0. However, this strategy has been criticized for being imprecise and for leading to an over retention of factors (Costello \& Osborne, 2005). The present study employed an alternative strategy by conducting a parallel analysis (Hayton, Allen, \& Scarpello, 2004), wherein a data set containing random numbers, but sharing similar parameters (i.e., the same sample size and number of variables), is compared against the obtained data set. The intended analyses are run on the random data, and the produced eigen values are recorded. The process is repeated many times (for the present research, 100 such replications were run), and the resulting eigen values are then tallied to calculate means and standard deviations. The resulting means and standard deviations are then used to calculate $95^{\text {th }}$ percentile values, which the factors produced by the real data must exceed in order to be retained. In theory, exceeding these $95^{\text {th }}$ percentile values is a demonstration that the retained factor accounts for variance above and beyond chance.

It was hypothesized that the proposed three-factor structure would not vary according to any demographic variables.

Study 2 


\section{Participants}

The present study utilized a convenience sample of undergraduate students at a local Midwestern university. Participants were offered the incentive of extra credit in their courses in exchange for participation. A posting for the study was placed within the aforementioned university's online study system. This posting offered a description of the study's purpose, length of time to participate, and amount of extra credit available. A total of 712 participants were obtained.

\section{Procedure}

Participants were informed that in exchange for completing a series of self-report measures assessing a number of domains, they would be eligible for extra credit. Participants then reviewed and signed a consent form and a complete the provided packet of measures. Of note, the procedure of study 2 is identical to that of study 1, utilizing the same methodology, and enjoying the same benefits (e.g., requiring participants to complete all of the requested items).

\section{Measures}

Relationship Questionnaire (RQ; Bartholomew \& Horowitz, 1991). The RQ is a self-report measure of adult attachment evaluating a four-type model of attachment styles. The four types are as follows: Secure, Fearful, Preoccupied, and Dismissive. The first half of the RQ contains a paragraph-long description of each of the four attachment styles. Based upon these descriptions, the respondent picks the attachment style that most accurately describes their experience in close relationships. In the second half of the RQ, participants rate how well the paragraphs describe their own experiences using a 7-point Likert scale with the following anchors: 1) Not at all like me;7) Very much like me. The RQ has demonstrated strong internal consistency and test-retest reliability (Roberts, Gotlib, \& Kassel, 1996). The RQ has been found 
to be significantly related to the attachment categories assigned by the Adult Attachment Interview (AAI), demonstrating agreement on classification $78 \%$ of the time (Simpson \& Rholes, 1998).

Narcissistic Personality Inventory (NPI; Raskin \& Hall, 1979). The NPI was derived from the DSM-III (APA, 1980) criteria for Narcissistic Personality Disorder.It consists of 40 items, presented as forced-choice dilemmas - with one option reflecting narcissistic tendencies. For instance, a respondent might be forced to choose between "I have a natural talent for influencing people" or "'I am not good at influencing people." The final score consists of the number of narcissistic selections endorsed. Scores can range from 0 to 40, with higher scores indicating greater degrees of narcissism. The NPI has demonstrated strong internal consistency (Raskin \& Terry, 1988), convergent validity (Emmons, 1984) and discriminant validity (Biscardi \& Schill, 1985).

Hypersensitive Narcissism Scale (HSNS; Hendin\& Cheek, 1997). The hypersensitive narcissism scale is a measure of covert narcissism adapted from Murray's (1938) 20-item Narcissism Scale. The HSNS consists of 10-items, using a 5-point Likert scale, wherein the respondent rates how "true" an item is for them. Items in the HSNS include the following: "I can become entirely absorbed in thinking about my personal affairs, my health, my cares, or my relations with others." In its initial validation study, Hendin \& Cheek (1997) found that the HSNS demonstrated adequate internal consistency, convergent validity, and discriminant validity. Such research has found that the HSNS is correlated with neuroticism (Hendin \& Cheek, 1997) an anxious attachment style (Besser \& Priel, 2009) and shyness (Gleason, Jarudi, \& Cheek, 2003). 
Rosenberg Self-Esteem Scale (RSES; Rosenberg, 1965). The RSES is a 10-itemselfreport measure of self-esteem. It is scored on a four-point Likert Scale, with answers ranging from 1 = strongly agree to 4 = strongly disagree. Higher scores are found to reflect higher levels of self-esteem. The RSES enjoys high test-retest reliability (Blascovich \& Tomaka, 1991).It is the most commonly used measure of self-esteem (Blascovich \& Tomaka, 1991).

Harder Personal Feelings Questionnaire (PFQ2; Harder \& Zalma, 1990). The PFQ2 is a 22-item instrument measuring the constructs of shame and guilt. It employs a 5-point Likerttype scale, with answers ranging from $0=$ Never to $4=$ Continuously or almost continuously. Scores on the shame subscale range from 0 to 40 , while scores on the guilt subscale range from 0 to 24. Higher scores indicate a greater level of proneness toward the given construct. Analyses of the PFQ2 have provided evidence of construct validity (Harder, Rockart, \& Cutler, 1993), internal consistency (Corcoran \& Fischer, 2000), and reliability (Harder \& Zalma, 1990).

Superiority and Goal Instability Scales (SGIS; Robbinson \& Patton, 1985). The SGIS is a 20-item measure of Kohut's developmental axes of grandiosity and idealization (with 10 items dedicated to each). Responses are made according to a 6-point Likert scale, with answers ranging from $1=$ Strongly Agree to $6=$ Strongly Disagree. The scales may be generally described as assessing the grandiosity (i.e., Superiority) and need for idealization (i.e., Goal Instability) poles of the self articulated by Kohut. The scales have demonstrated adequate reliability and convergent validity (Robbins, 1989). The scales are described in greater detail previously.

Social Connectedness Scale-Revised (Lee, Draper, \& Lee, 2001). The Social Connectedness Scale is a 20-item measure of interpersonal closeness designed to tap into Kohut's third pole of development: connectedness. The measure assesses the degree of 
interpersonal closeness experienced by the respondent, as well as the degree of difficulty in maintaining this closeness. Responses are made according to a 6-point Likert scale, with answers ranging from $1=$ Strongly Agree to $6=$ Strongly Disagree. The scale is an update of The Lack of Connectedness Scale (Lee \& Robbins, 1995), which itself demonstrated strong reliability and convergent validity (Lee \& Robinson, 1998). The updated scale has also demonstrated strong psychometric properties (Lee et al., 2001). The scale is described in greater detail above.

Brief Symptom Inventory (BSI; Derogatis \& Melisaratos, 1983). The 53-item BSI is a revised and shortened version of the Symptom Checklist 90 Revised (SCL-90-R). It covers nine clinical areas: somatization, obsessive-compulsive, interpersonal sensitivity, depression, anxiety, hostility, phobic anxiety, paranoid ideation, and psychoticism. The BSI has demonstrated strong internal consistency reliability (Derogatis \& Melisaratos, 1983) and convergent validity( Recklitis and Rodriguez, 2007).

Selfobject Needs Inventory (SONI; Banai, Shaver, \& Mikulincer, 2005). The SONI is a 38 item self-report measure, with answers ranging from $1=$ Not at All to $7=$ Very Much. Five factors derived from Kohut's theory of selfobject needs were identified: hunger for twinship, avoidance of idealization, twinship needs, hunger for idealization, hunger for mirroring, and avoidance of mirroring needs. Factor structure, test-retest reliability, and concurrent and divergent validity were also investigated during the development of the measure, and were found to be acceptable. The scale is described in greater detail above.

Paulhus Deception Scales (PDS; Paulhus, 1998). The PDS is a 40-item self-report measure designed to assess the respondent's tendency to provide socially desirable responses, even if such responses do not align with the respondent's true feelings. The PDS is unique in 
that it assesses two distinct forms of social desirability in the responding: self-deception and impression management. Self-deception is believed to represent an unconscious defense against potentiality threatening beliefs or feelings, while impression management is seen as a conscious distortion to make oneself appear better. The measure, and the validity of its two-factor design, has been supported in several reviews (e.g., Lanyon \& Carle, 2007).

Analyses

This second sample allowed for two critical analyses. First, a confirmatory factor analysis was conducted on the new measure. The factor structure identified in the first study, a proposed three-factor model, was examined. A wide range of fit statistics were considered, including: chi-square, the comparative fit index (CFI), the standardized rootmean-square residual (SRMR), and the root-mean-square errorof approximation (RMSEA). These fit statistics offered an important test to examine the measure's theorized structure.

Second, disrciminant, cluster, and correlational analyses between the factors of the new measure and the constructs assessed congruently were examined. These analyses placed the new measure in a larger nomological network, thereby providing evidence of the measure's convergent and discriminant validity. 


\section{CHAPTER IX}

\section{RESULTS}

$\underline{\text { Study } 1}$

\section{Item Generation}

A team of five raters evaluated the list of potential items generated for the present study. A total of 51 potential items were generated for the selfobject need of idealizing, 43 potential items were generated for the selfobject need of mirroring, and 42 potential items were generated for the selfobject need of twinship. Average ratings of clarity and relevance were then calculated for each potential item.

Based upon the criteria of an item needing a mean score of " 3 " in both clarity and relevance, items were deleted from each category of selfobject needs. In this initial analysis, 37 Idealizing items were retained, 24 Mirroring items were retained, and 15 Twinship items were retained.

Idealizing items were then trimmed to avoid redundancy and ambiguous language. Items with the lowest scores were also targeted for removal. This process further reduced the number of Idealizing items to 25 .

To increase the number of Twinship items, items that received high scores on relevance but insufficient scores on clarity were targeted for improvement. These re-worded items were then rated by a subset of three raters for clarity only (again requiring a mean rating of 3 ). Based upon these additions, 21 Twinship items were ultimately retained.

As can be seen in Table 1, on the selfobject need of idealization, there were 22 instances when raters produced significantly discrepant ratings (i.e., at least one individual rated the item as worse than acceptable, while another rater described the item as better than acceptable). 
Additionally, there were 22 instances of similarly discrepant ratings for the selfobject need for mirroring, and 19 instances on the selfobject need for twinship.

The original items and their expert ratings are presented in Tables 1-3.

The final potential items for the AESOP are presented in Appendix A. Exploratory Factor Analysis

The 70 remaining items of the present scale were subjected to a maximum likelihood exploratory factor analysis. A total of 738 participants completed the measure. Upon review of the responses, 52 respondents were removed from the analysis. These participants' data were removed if the respondent reported an age of under $18(n=3)$, if the respondent completed the present measure in less than four minutes (a four minute completion time placed these respondents in the $99^{\text {th }}$ percentile of completion time; $n=43$ ), or if all responses were identical or nearly identical (e.g., responding with all 1's; $n=6$ ). This reduced the present sample to 686 respondents.

Prior to performing the factor analysis the suitability of the data for the factor analysis was assessed. Inspection of the correlation matrix revealed the presence of many coefficients of .3 and above. The Kaiser-Meyer-Oklin value was .93, exceeding the recommended value of .6 (Kaiser, 1974) and the Bartlett's Test of Sphericity (Bartlett, 1954) reached statistical significance. These results suggest that a factor analysis is appropriate for the present data.

Based upon pre-established criterion, items were deleted under the following conditions: significant loadings on multiple factors, failure to load significantly on a single factor, item-total correlations of lower than .4 with items on the same factor, and if the item's removal would result in an increase to Cronbach's alpha. Items were viewed to load significantly on a factor if the factor loadings were .32 or above (Tabachnick \& Fidell, 2001). Based upon these criteria, 11 
mirroring items were deleted, 15 idealization items were deleted, and 13 twinship items were deleted.

Following the removal of these items, the underlying factor structure of the measure was reviewed. A parallel analysis with 100 replications was conducted utilizing a sample size of 686 respondents and 31 variables. Based upon this analysis, three factors were retained, with eigen values of $8.53,3.97$, and 1.87 . These values exceeded the $95^{\text {th }}$ percentile eigen values produced in the parallel analysis $(1.42,1.36$, and 1.32$)$. The fourth eigen value produced by the parallel analysis (1.29), was not exceeded. Review of the produced scree plot also suggested the retention of three factors. These three factors accounted for $27.5 \%, 12.8 \%$, and $6 \%$ of the variance, respectively.

To aid in the interpretation of these factors, a direct oblimin rotation was performed. The rotated solution is presented in Table 4. The remaining items were found to sort into the predefined categories of the three selfobject needs. As can be seen, the strength of these loadings was highly variable.

All three of the scales produced acceptable reliability. The 10-item Idealizing scale produced a Cronbach's alpha of .79, the 13-item Mirroring scale produced a Cronbach's alpha value of .91 , and the 8 -item Twinship scale produced a Cronbach's alpha of .80 . The various item-total correlations and the "Cronach's alpha if item were deleted" statistics are presented in Table 5.

Study 2

\section{Confirmatory Factor Analysis}

Method. A total of 712 participants completed the measure. Upon review of the responses, 40 respondents were removed from the analysis. These participants' data were 
removed if the respondent reported an age of under $18(n=0)$, if the respondent completed the present measure in less than 15 minutes (a 15 minute completion time placed these respondents in the $99^{\text {th }}$ percentile of completion time; $n=32$ ), or if all responses were identical or nearly identical (e.g., responding with all 1's; $\mathrm{n}=8$ ). This reduced the present sample to 672 respondents.

The factor structure that was identified with the EFA from Sample 1 was confirmed in Sample 2 using a latent modeling technique. The present items were entered into a confirmatory factor analysis (CFA) using Mplus (v. 7, Muthen and Muthen, 2012). Measurement items were allowed to intercorrelate within a factor, but were restrained to not correlate or load across factors. For the purpose of the CFA the scale ratings were treated as a true interval scale. Ordinal Likert scales are often interpreted as a continuous variable, and so to better evaluate its use as such, the present measure's factors were confirmed using a maximum likelihood estimation method. Model fit was evaluated with a compendium of accepted fit indices (Raykov \& Marcoulides, 2006; Hu \& Bentler, 1999): normal theory weighted chi-square statistic (a nonsignificant value indicates good fit); root mean square error of approximation (RMSEA, a value less than 0.05 indicates excellent fit); comparative fit and Tucker-Lewis fit indices (CFI and TLI, respectively, values exceeding 0.90 indicate excellent fit); standardized root mean residual (SRMR, less than 0.08 supports adequate fit).

Results. The factor structure identified from the EFA in Sample 1 was confirmed in Sample 2. All measurements significantly identified the respective latent factor (all $p<0.001$ ). See Table 6 for a list of factor loadings. The latent model fit well: $\mathrm{RMSEA}=0.046, \mathrm{CFI}=0.93$, $\mathrm{TLI}=0.91$, and SRMR $=0.07 . \quad$ The chi-square test was significant $\left(\chi^{2}(388)=950.39, \mathrm{p}<\right.$ 0.001), which may be a biased statistic give the large sample size and number of parameters. 
Nonetheless, the analysis confirmed the factor structure in a second independent and randomlyselected sample. The latent factors were moderately intercorrelated: $0.38-0.60$, all $\mathrm{p}<0.001$.

Scale responses did not vary by age $(F=1.36, p=.26)$, sex $(F=1.8, p=.17)$, or ethnicity $(\mathrm{F}=1.44, \mathrm{p}=.19)$.

Discriminant Analysis: Predicting Psychological and Affective Outcomes

Methods. Several personality attributes and psychological health outcomes were measured in Sample 2. These outcomes were dichotomized within the sample to represent highscoring individuals (i.e., the top $25 \%$ of the included measures) and the remaining $75 \%$ of the sample. Overt (NPI) and covert (HSNS) narcissism, RSES, idealization and grandiosity (SGIS), and social connectedness (SCS) were dichotomized at the $75^{\text {th }}$ percentile: those in the upper $25 \%$ of the sample were classified as the clinical group, and all others as normal. The several Brief Symptom Inventory scales have published t-values for non-patient men and women. For all scales a $t \geq 65$ was classified as a clinical group, and below this criterion as a normal group. The AESOP scale totals were entered as correlated predictors into a discriminant model for each dichotomous outcome. Model fit was evaluated by Wilk's lambda and estimation classification accuracy. Cannonical correlations were converted to $\mathrm{R}^{2}$ statistics as an estimate of effect size. To correct for multiple comparisons, a more conservative alpha criterion was used, $\mathrm{p}<0.01$. Differential effects between the present measure's scales in predicting the outcomes were evaluated with a Steiger Z comparison of bivariate correlations (Steiger, 1980). The Steiger Z accounts for the correlation between the present measure's scales and a significant value indicates a difference between scales in relationship to an outcome.

Results. The AESOP's scales discriminated between high-scoring individuals (i.e., the top $25 \%$ of the included measures) and the remaining $75 \%$ of the sample. See Table 7 for a 
summary of all discriminant analyses. Discriminant accuracy was good for all outcomes: 67.1$78.6 \%$, indicating that on average, fewer than 3 out of 10 cases were misclassified by the present scales. Notably, the present scales were most sensitive to covert narcissism $\left(R^{2}=0.21\right)$, but were not strongly associated with overt narcissism $\left(\mathrm{R}^{2}=0.02\right)$.

The three scales did not equally predict each outcome. See Table 8 for a simplified report of the scale prediction of each outcome. The Mirroring scale was the most common correlate of the various outcomes, and was more strongly associated with the outcome when more than one scale was a predictor (all Steiger $Z>11.5$, all $p<0.001$ ), except for grandiosity and the NPI.

The Twinship scale was associated with more of the outcomes than the Idealizing scale. When both scales were covariates, the Twinship scale was more strongly associated with Hypersensitive Narcissism Scale $(\mathrm{p}<0.01)$, interpersonal sensitivity $(\mathrm{p}=0.01$ ), and paranoid anxiety $(\mathrm{p}=0.01)$; and was overlapping with Ideal in predicting anxiety, obsessive compulsive behavior, and grandiosity (all $\mathrm{p} \geq 0.05$ ).

\section{Cluster Analysis: Observed Clusters within Sample 2 on the Present Measure and Outcomes}

Method. As a follow-up to the discriminant analysis that identified predictability of outcomes by the AESOP's scales, a cluster analysis determined the patterns of covariance observed in the sample. In other words, the analysis identified subgroups of respondents with different combinations of scale responses and then determined group differences in outcomes. Thus, the three present measure's scales were entered into a two-step cluster analysis as continuous variables (log-likelihood distance estimation, Schwarz's bayesian criterion), and evaluated for differences in the outcome measures. Correction for multiple comparisons was made by using a more conservative $\mathrm{p}$-value, $\mathrm{p}<0.001$. 
Results. Two clusters were observed with fair separation (average silhouette $=0.40$ ): those who responded lower on the 3 scales $(n=252,37.5 \%)$ and those who responded higher $(n$ $=420,62.5 \%$ ). All three scales discriminated between the clusters (predictor importance $\geq$ 0.56), but the Twinship scale was the most sensitive to individual differences (predictor importance $=1.0$ ). A post-hoc univariate ANOVA confirmed that the groups significantly differed on all outcomes (all $\mathrm{F} \geq 8.24$, all $\mathrm{p}<0.001$ ), except for NPI $(\mathrm{p}=0.045)$ and social connectedness $(\mathrm{p}=0.004)$ that were non-significant after correction for multiple comparisons. The groups differed most strongly on Hypersensitive Narcissism Scale, followed by grandiosity and several Brief Symptom Inventory scales (see Figure 1 for a comparison of outcomes between groups).

Figure 1.

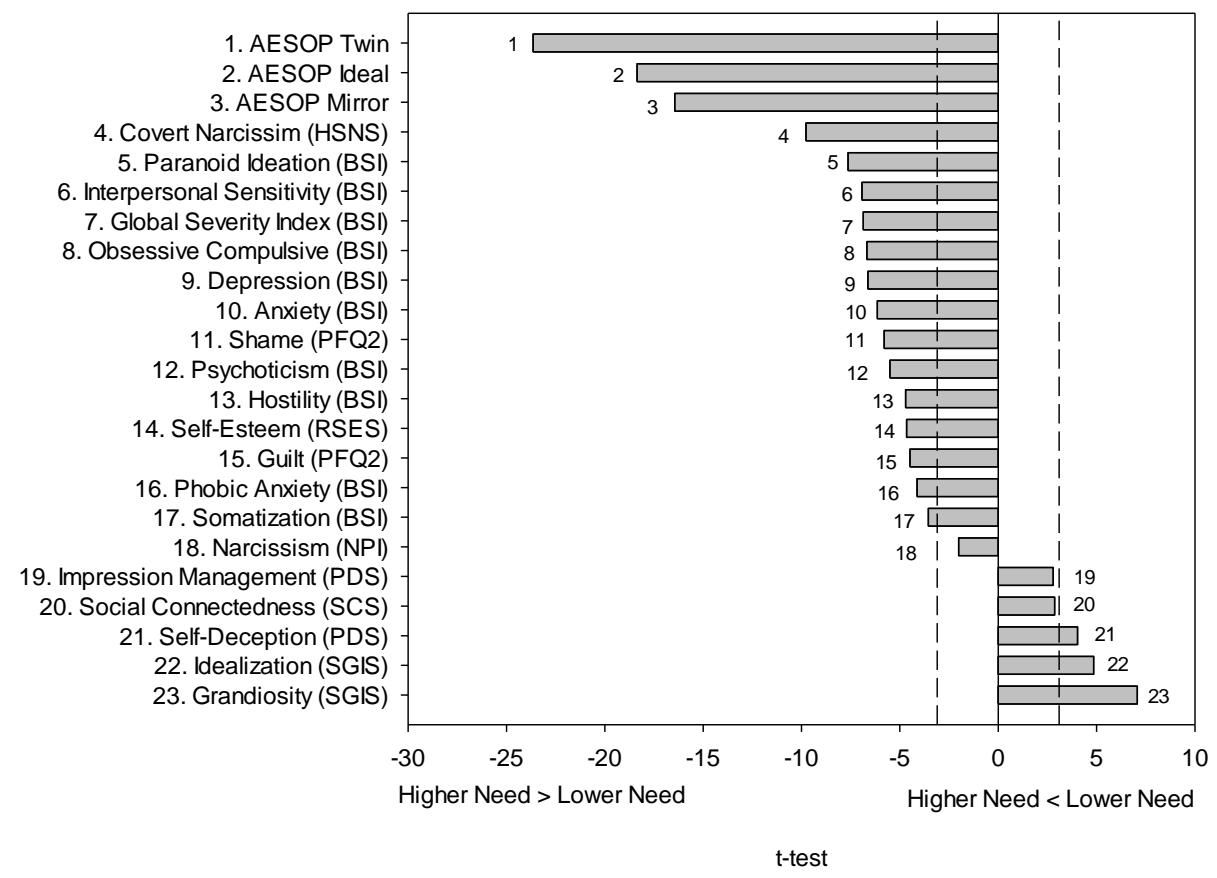

Note: Approximately $63 \%$ of the sample endorsed higher need on all c scales than did the remainder of the sample. Bars represent t-tests of cluster group comparisons. Dotted lines are critical $t$ values for significance at $\mathrm{p}<0.001$. 
Confounding Variable Analysis

Self-deception and impression management. The present measure's scales were negatively correlated with PDS scales. Mirroring was negatively correlated with both selfdeception $(\mathrm{r}=-0.38, \mathrm{p}<0.001)$ and impression management $(\mathrm{r}=-0.25, \mathrm{p}<0.001)$, while Twinship was negatively correlated only with the former $(r=-0.12, p<0.01)$ and not the latter $(r$ $=-0.06, \mathrm{p}=0.11)$. Idealizing was unrelated to either $(\mathrm{r}=0.03$ and -0.002 , respectively, $\mathrm{p} \geq$ 0.38). In addition, after correction for multiple comparisons, Cluster 1 (low responses) was significantly higher in self-deception, but there was no difference between clusters in impression management.

Attachment style. A repeated measure MANOVA tested for differences in the present measure's scale ratings between attachment style groups. The group differences varied by the scale $(\mathrm{F}=7.01, \mathrm{p}<0.001)$. Post-hoc with $95 \%$ confidence intervals $(\mathrm{CI})$ identified that differences were isolated to the Mirror scale $(\mathrm{F}=14.26, \mathrm{p}<0.001)$, which was lower in the dismissing attachment style $(\mathrm{M}=40.00, \mathrm{SD}=15.17 ; 95 \% \mathrm{CI} 37.08 / 42.92)$ compared to fearful attachment $(\mathrm{M}=52.34, \mathrm{SD}=15.90 ; 95 \% \mathrm{CI} 49.36 / 55.55)$. Groups did not differ on Idealization $(\mathrm{F}=0.97, \mathrm{p}=0.41)$ or Twinship $(\mathrm{F}=2.46, \mathrm{p}=0.06)$ scales.

\section{SONI Factor Structure}

Given the interest of the SONI in contrast to the AESOP, particular attention was given to the SONI's underlying factor structure. A total of 712 participants completed the measure. Upon review of the responses, 40 respondents were removed from the analysis. These participants' data were removed if the respondent reported an age of under $18(\mathrm{n}=0)$, if the respondent completed the present measure in less than 15 minutes (a 15 minute completion time placed these respondents in the $99^{\text {th }}$ percentile of completion time; $n=32$ ), or if all responses 
were identical or nearly identical (e.g., responding with all 1's; $n=8$ ). This reduced the present sample to 672 respondents.

A CFA on the structure published for the SONI scale was conducted in Mplus. Latent model fit for a 5 factor structure was acceptable, but poor: $\chi^{2}(124)=2623.21, \mathrm{p}<0.001$; $\mathrm{RMSEA}=0.07 ; \mathrm{CFI}=0.79$ and TLI $=0.78 ; \mathrm{SRMR}=0.08$. All items significantly loaded on the respective factors; however, more than half of the items significantly cross-loaded onto more than one factor. Model fit could be significantly improved if 17 of the 38 items were allowed to cross-load on 3 or more factors, and an additional 9 items to cross-load on 2 factors. A second quandary was the pattern of correlations amongst the factors. Factor 1 was correlated with Factor $3(\mathrm{r}=0.75, \mathrm{p}<0.001)$ and Factor $4(\mathrm{r}=0.54, \mathrm{p}<0.001)$. Factor 2 was positively correlated with Factors 3-5 (all $\mathrm{r} \geq 0.10, \mathrm{p}<0.05$ ), and Factors 4 and 5 were negatively correlated $(r=-0.39, \mathrm{p}<0.001)$.

To further examine the SONI's underlying factor structure, the items of the SONI were subjected to a maximum likelihood exploratory factor analysis, thereby repeating the analyses conducted on the present measure. A parallel analysis with 100 replications was conducted utilizing a sample size of 672 respondents and 38 variables. Based upon this analysis, four factors were retained, with eigen values of $8.05,5.17,2.87$, and 1.63 . These values exceeded the $95^{\text {th }}$ percentile eigen values produced in the parallel analysis $(1.42,1.36$, and 1.32$)$. The fifth eigen value produced by the parallel analysis (1.29), was not exceeded. Review of the produced scree plot also suggested the retention of four factors. These four factors accounted for $21.2 \%$, $13.6 \%, 7.5 \%$, and $4.3 \%$ of the variance, respectively.

To aid in the interpretation of these factors, a direct oblimin rotation was performed. The rotated pattern matrix is presented in Table 9. As suggested in the CFA, there were numerous 
cross-loadings of SONI items across the four factors. Furthermore, the groupings of the various items appeared discrepant from those posited during the SONI's development.

\section{Comparison to SONI}

Methods. All analyses with the AESOP were repeated for the SONI measures. Comparisons to the present measure were made within each analysis with Steiger Z, controlling for the correlation between the present measure's scales and the corresponding SONI positive attribute scales.

Results. In the discriminant analysis, SONI (5 scales) identified the clinical group from the normal on all variables (all Wilk's $\lambda=0.81-0.93$, all $\mathrm{p}<0.001$; all canonical $\mathrm{r}=0.27-0.44$ ) with good accuracy (65.5-79.8\%). In comparison to the present measure, SONI was comparable on almost all outcomes. Comparatively, SONI was more strongly associated with the NPI (Steiger $Z=-5.16, p<0.001)$ and Brief Symptom Inventory phobia (Steiger $Z=-2.76, p=0.01$ ); whereas the present measure was stronger on Brief Symptom Inventory paranoia (Steiger Z = $2.41, \mathrm{p}=0.01)$. However, examination of the direction of significant predictors, the IdealizationTwinship Avoidant scale was consistently in the same direction as the approach scales, which is inconsistent with the original scale construction.

This inconsistency was echoed in the cluster analysis. Two clusters were identified with fair separation (average silhouette $=0.40$ ): cluster 1 was lower on all approach and the IdealTwinship avoidant scales, and cluster 2 was higher on these 4 scales. Most notably, however, was that the two avoidant scales had very low predictor importance $(0.20$ and 0.01$)$, and were therefore not distinguishing individual differences well. Indeed, further analysis of the outcomes indicated that Hypersensitive Narcissism Scale (predictor importance $=0.34$ ) was a stronger discriminant between the groups than either avoidant scale. In addition, the SONI groups 
differed largely on both impression management and self-deception, both of which may be confounds to the scale. Finally, SONI differed between attachment styles $(\mathrm{F}=4.88, \mathrm{p}<0.001)$ : dismissing attachment style was lower on Mirror-Approach as compared to the other groups $(\mathrm{F}=$ 23.54, $\mathrm{p}<0.001$ ), and fearful attachment was higher on Twin-Ideal-Avoidant than secure $\operatorname{attachment}(\mathrm{F}=4.81, \mathrm{p}=0.003)$.

Correlations of all included variables are presented in Table 10. 


\section{CHAPTER X}

\section{DISCUSSION}

The present research was focused on the creation of an empirically-supported measure of the abstract, but broadly employed, concept of selfobject needs. This new measure was designed through a multi-step process involving expert clinical raters generating and rating the face validity of the items, an exploratory analysis of potential items among a relatively diverse sample of 686 college students, an independent replication and statistical confirmation of the measure's proposed structure among a different, diverse sample of 672 college students, and an examination of its relationship with other constructs of interest for the second sample. Following these procedures, an apparently clean and stable structure aligning with the proposed measure's three factor-structure emerged. Additionally, the relationships of this measure to other included measures offers evidence of the measure's convergent and discriminant validity. In the discussion to follow, the specific and overall implications of these findings will be addressed. The latter parts of the discussion addresses findings that challenge the measure, other limitations, and suggestions for future research.

\section{Item Generation}

The process of developing items for the associated selfobject needs emerged after a broad survey of Kohut's original source material (e.g., Kohut, 1971), related self psychology research (e.g., Tolpin, 2002), diverse literary sources (e.g., Sartre, 2007), and consultation with clinical providers familiar with self psychology theory and practice. A liberal approach was taken toward the generation of items, under the logic that subsequent review (both by raters and statistical analysis) could remove unneeded items, while a lack of a sufficient number of items would require a more onerous correction. All items generated had a straightforward, face valid 
connection to feelings and behaviors described by Kohut's descriptions of the selfobject needs demonstrated in the therapeutic transference..

As mentioned previously, a total of 51 potential items were generated for the selfobject need of idealizing, 43 potential items were generated for the selfobject need of mirroring, and 42 potential items were generated for the selfobject need of twinship. The items for idealizing proved to be the easiest to generate for the dissertation team, as it yielded the highest number of potential items as well as the greatest rate of retention. Twinship proved to be the most difficult, as the number of items retained and the retention rate of its items was the lowest among the three.

Kohut's $(1971,1977,1984)$ description of the selfobject needs, and his general theory of psychopathology as following from a model wherein the lack of selfobject need fulfillment results in a variety of clinical presentations, is both challenging and abstract, offering a reasonable explanation for the difficulty in generating successful items. Furthermore, that the Twinship need proved the most difficult in this respect, appears reasonable from a theoretical perspective. Twinship was the last need identified by Kohut (1984), as it had initially been subsumed under the need for Mirroring. Others went on to challenge Kohut's positing of a separate Twinship need has also been challenged by other authors, some of whom regard Twinship as a more of an underlying need behind all selfobject needs, rather than an independent selfobject need (e.g., Basch, 1992). Nonetheless, the exploratory, confirmatory, and cluster analyses in the present research (to be discussed subsequently), as well as the ratings provided by expert reviewers, supports the conceptualization of Twinship as an unique, independent need.

Despite these potential difficulties, over 20 items receiving mean relevance ratings of "Acceptable" or better among five independent experts in self psychology were generated for 
each selfobject need. At a fundamental level, the ability to create such a list of items obtaining expert consensus suggests a certain theoretical unity among the constructs. Furthermore, the created items were intentionally kept distinct from related, but potentially tautological symptoms derived from unmet selfobject needs. For example, unmet Idealizing needs are posited to result in a potentially anxious disposition. Accordingly, a potential item such as "I feel anxious in new social situations" would theoretically expect to be endorsed by an individual with an unmet need for Idealization. However, use of such items already includes the self-object need linked with the symptom anxiety and therefore would inflate the size of the positive relation between selfobject need with measures of anxiety. Thus, the present items were designed to eliminate this built in confound with symptoms.

Conversely, the Mirroring item “I often feel like people don't appreciate the struggles I've had to overcome," specifically addresses a common experience of individuals who have been insufficiently mirrored. While such an item may indeed be endorsed by a depressed individual, there is inherently nothing about depression that would require such an experience. A depressed individual might report feeling as though they haven't overcome any struggles, or that they don't deserve such appreciation. Thus, the present measure's items attempted to avoid symptom confound sometimes observed in other self-report measures. The agreement of raters on a set of items of these abstract constructs, particularly while avoiding reference to obvious symptoms, offers an initial endorsement of the coherence of the construct.

Exploratory Factory Analysis

The theorized three-factor structure of the AESOP aligning with the selfobject needs of Twinship, Mirroring, and Idealizing was supported by the results of the initial exploratory factor analysis. Although several items had to be deleted due to significant cross-loadings or failure to 
load significantly on a single factor, this was not unexpected based upon the aforementioned liberal approach taken towards item generation. As in the item generation phase, the fewest number of items were retained by the Twinship factor, as only eight items met all of the required prerequisites for retention (as compared to 13 and 10 items for Mirroring and Idealizing, respectively).

A neatly defined three factor structure was supported by the final results, with each of the items loading significantly onto its respective selfobject need. The strength of these loadings was somewhat variable, ranging from .34 to .77 , though all exceeded the criterion value of .32 (Tabachnick \& Fidell, 2001). The sorting of these values into the three-factor structure is of great significance, as it provides an empirical demonstration of the potential distinction between the proposed selfobject needs, and supports Kohut's proposed three selfobject needs.

An initial question of interest was if selfobject needs can be reasonably expected to sort into separate factors. Although each need is theoretically distinct, the experience of selfobject needs may also be described as inherently entwined. For example, if a child is to derive a sense of value and enhancement from the experience of being mirrored, the mirroring parent should be idealized, at least to some extent. In a similar vein, the experiencing a sense of twinship is best facilitated through receiving mirroring from the intended "twin." Based upon this relatedness, the potential for the items to not sort into the pre-defined categories was legitimate.

In the present analysis, all of the respective selfobject needs were correlated (ranging from .31to .44), though none of the values were so highly correlated as to convey a sense of redundancy among the needs. This significant positive correlation between the factors, combined with the sorting of the variables into the three posited factors without overlap, instead supports the original contention. Namely, that the individual selfobject needs are best 
conceptualized as distinct but related concepts. Furthermore, it appears to follow that a deficit in one selfobject need may not necessitate a deficit in the related needs.

\section{Confirmatory Factor Analysis}

A large sample exploratory factor analysis is an important beginning for the development of the AESOP. At a minimum, it demonstrates that a theoretically derived measure of the intended constructs can be created. Furthermore, it offers preliminary support for the notion of separating the proposed selfobject needs into three categories. However, the stability for such a measure is highly uncertain. Exploratory analyses represent only an initial step in a larger validation process. Due to constraints within the modeling process, confirmatory and exploratory analyses often produce discrepant results (Prooijen \& Kloot, 2001). As such, an exploratory analysis cannot be assumed to produce a stable and enduring pattern. Furthermore, an initial EFA is a solitary effort in need of confirmation and replication.

The strong results of the confirmatory factor analysis thus offer crucial supplementary evidence for the validity of the measure's proposed three-factor model. All of the fit indices suggest an adequate to strong model fit, with the exception of the significant chi-square value. This finding is unsurprising, as with the large number of respondents, some researchers have suggested that it is more appropriate to test model fit with a normed chi-square (Bollen, 1989), with values less than 0.3 indicating a good model fit. Under this criterion, the present model's chi-square value is also indicative of good model fit (Kline, 2005). In short, the three factor model appears supported in present analysis.

That the three factor structure was hypothesized based upon theoretical grounds, was identified in an exploratory factor analysis, and then confirmed in an independent sample, offers strong grounds for concluding that the proposed model enjoys notable support. The three scales' 
reliability ranged from acceptable to excellent, again suggesting the feasibility of the three-factor model. In this regard, the Idealizing scale reports the lowest internal consistency (Cronbach's alpha $=.79$ ), falling a mere .01 away from typical standards of "good" reliability (Kline, 2000).Speaking summarily, the measure's proposed structure has received consistent statistical and theoretical support.

SONI

The most relevant comparative instrument to the AESOP is the SONI, a measure also designed to tap Kohut's selfobject needs (though it employs an approach/avoidance axis absent from the current measure). As mentioned previously, the SONI has not demonstrated a consistent structure across subsequent validation studies (e.g, Bastanfar, 2009). Indeed, the author's original six-factor structure was not supported in the SONI's foundational study, instead resulting in a five factor model. Given the potential overlap of the SONI with the present measure, the underlying factor structure of the SONI was also examined in the present sample.

The confirmatory factor analysis testing the model proposed by the SONI's authors suggested a poor fit, with cross-loadings across the five proposed factors being a persistent issue. This same difficulty has arisen in previous studies with the SONI, suggesting that the model's organization is somewhat precarious. Fit indices ranged from poor to acceptable, again questioning the structure of the SONI.

In parallel to the present measure, an exploratory analysis with the SONI was also conducted. In this analysis, the SONI presented a muddled organization, with a four factor solution. As can be seen in Table 9, the four factor model is highly cluttered by numerous crossloadings. The first factor consists only of approach-orientation items, though items from each of three selfobject needs were found to significantly load on the factor. More distressingly, in the 
remaining three factors, both approach and avoidance items were found to demonstrate significant loadings. This raises questions, not only about the SONI's organization, but also about the distinction it purports to make regarding the approach-avoidance axes.

That the SONI's structure faired so poorly in the present analysis raises significant questions regarding the measure's validity. While the individual items of the SONI seem to derive from a theoretically sound methodology, the methods employed to create the measure appear lacking. In the SONI's development, the primary source underlying the measure's structure was a factor analysis with a varimax rotation. As has been mentioned, an initial factor analysis represents only an initial developmental step, and thus subsequent replication of the measure's psychometrics was missing. Additionally, the failure of the SONI to produce the intended factor structure was an immediate cause for concern. Several specific shortcomings may account for the measure's present difficulties.

First, factor retention was based upon the number of factors with eigen values exceeding one. There has been a documented history of criticism of this approach, with it being widely regarded as one of the least accurate methods of factor retention (Velicer \& Jackson, 1990). Monte Carlo analyses using this method have been found to regularly suggest an over-retention of factors (Costello \& Osborn, 2005). Second, the authors employed an orthogonal rotation method, which despite its ability to provide a sense of clarity among the factors, yields a solution based upon the false assumption that the factors will be uncorrelated. Given the nature of the reported factors, such a rotation method appears ill-advised. Finally, no confirmatory analysis on the proposed structure was conducted. The importance of a CFA is highlighted by the statistical differences between it and a traditional factor analysis. Because the number of factors in a CFA 
must be specified in advance, there is a resulting restriction of parameters not seen in the original factor analysis, thereby leading to potentially divergent results.

The cross-loadings and differing factor structure make interpretation of the SONI's relationships highly difficult. Though the specific items of the SONI likely bear more than a minor relationship to items of the present measure, variance attributable to the SONI is hard to define. Namely, it is difficult to know if the SONI is best conceived as an overall measure of selfobject neediness, as opposed to reflecting three separate measures.

\section{Cluster and Discriminant Analyses}

The present measure was confirmed to have three distinct and correlated scales. Although each object need may be related to a common source in one's personal history, the psychological consequences of each need are suggested to differentially reflect distinct personality features. This intriguing hypothesis was supported by the discriminant analysis that tested each scale as a predictor of personality functioning. Interestingly, the Mirroring subscale was the strongest correlate of the several personality and psychological symptom measures. The direction of the association was consistent with Kohut's original description of the selfobject need construct - persons who endorsed a high frequency of Mirroring needs were commonly in the upper $25^{\text {th }}$ percentile of covert narcissism, shame, and reported clinical levels of symptoms on the Brief Symptom Inventory. However, the scale was not selective, correlating with all measures, except for the Narcissistic Personality Inventory. The Idealizing and Twinship scales were more selective in correlating with outcomes. Higher Idealizing and Twinship needs were associated with higher covert narcissism, and of the two scales, Twinship was the stronger predictor. Indeed, the Twinship scale was more sensitive to individual differences in needs than the other two scales. In addition to narcissistic tendencies, high Twinship needs also identified 
persons who reported clinically significant levels for several psychological outcomes, including anxiety, phobia, and obsessive compulsive behaviors. Whereas the Mirroring and Twinship scales were correlated with most of the Brief Symptom Inventory psychological scales, high Idealizing was only associated with reporting more psychotic symptoms. The only convergence across all three subscales was in predicting covert narcissism.

Consistent with Kohut's original conceptualization of selfobject needs, high need was associated with a greater frequency of narcissistic personality function. Kohut posited that while higher selfobject needs would be associated with narcissism generally, Kohut's description of narcissism is the basis of the disorder's theorized covert form; a personality presentation containing a hidden sense of grandiosity, combined with a shy and avoidant outward demeanor (this is contrasted against the presentation of narcissism in its overt form, emphasizing an outward sense of arrogance and grandiosity). Thus a more direct relationship between selfobject needs and covert narcissism would be expected, and this indeed appears to be the case. Only the Idealizing subscale was associated with overt narcissism, whereas all three scales were predictive of covert narcissistic tendencies. For all scales, reports of higher object need were associated with an increased likelihood of the person being in the upper $25^{\text {th }}$ percentile. The non-specific association between Idealizing need with both overt and covert narcissism may suggest that the Idealizing subscale can be used as a general screening measure for narcissism. Thus, the subscales appear to have unique predictive utility to differentiate between personality functions and psychological symptoms.

The subscales were indeed confirmed as unique factors with differential predictive use, but importantly the subscales were moderately inter-correlated. Again, consistent with Kohut's original definitions of the object need constructs, the three sources of needs are independent and 
related. Therefore, study of the scales in isolation may be informative as to the unique source of the behavior, but the three scales should also function as mutually diagnostic.

The complimentary use of all three scales as a single measure for clinical diagnosis was confirmed with a cluster analysis. The sample of college students naturally diverged into two groups, with one cluster (approximately $63 \%$ of the sample) providing significantly higher scores on all three selfobject needs than the remaining cluster (of note, this significance refers to differences between the groups, not absolute values on the scale itself). The two clusters did not differ in average age $(t(669)=1.54, p=0.12)$, nor in the number of women $\left(\chi^{2}=0.07, p=0.79\right)$ or in ethnic distribution $\left(\chi^{2}=8.66, \mathrm{p}=0.28\right)$, suggesting there is no inherent bias in the scales. Based on the measure's-defined groups, persons with high need tended to have an increase in covert narcissism, higher interpersonal sensitivity, and an increased risk for the several psychological outcomes. Whereas high need was associated with higher narcissism, low object need was associated with a higher sense of grandiosity, possibly interpreted as a greater sense of self-confidence. Therefore, the direction of the scale values is potentially informative for diagnostic screening.

Whereas high need may lead to maladaptive behaviors, the meaning of low needs is more uncertain. Such low needs may be associated with greater self-reliance and confidence, or perhaps low needs reflect a defensive reaction. Indeed, as suggested by the cluster analysis, the lower scoring cluster produced significantly higher scores on the included measure of selfdeception (though not impression management). As one might expect, an individual who seeks to deny their own needs, will likely not be inclined to endorse such items on a self-report measures. In either case, the interpretation of these low values requires additional empirical study. The present measure was intentionally constructed to allow for interpretation of low 
values as low need, not necessarily as high avoidant behavior; thereby allowing for the possibility that low need was associated with more adaptive behaviors. Of note, approximately one-third of the present sample was classified as having lower need.

This is an important consideration, given that a critical feature of this scale development was a comparison with the SONI scale of selfobject needs. The SONI's structure could not be confirmed in this sample, suggesting measurement invariance that undermines the interpretation of the scale. This may be in part due to the construction of both approach and avoidant scales, as described before. The avoidant scales were either ineffectual, or were correlated in the same direction as the approach scales, counterintuitive to the original scale construction. In comparison, the three factor scale of the present measure was confirmed in a second, randomlyselected sample. Further, the present scale was confirmed in a nomological network in relation to narcissistic personality function and psychological symptoms. The SONI also correlated with these outcomes, though high approach behaviors were more strongly associated with covert and overt narcissism than either of the avoidant behavior scales, suggesting a potential shortcoming in the SONI's organization (namely, that the avoidance scales appear to contribute little). Further, SONI responses were highly correlated with potential confounds of impression management and self-deception. Therefore, the SONI scale items may be biased towards detecting narcissism and may omit other relevant dimensions of selfobject needs.

Taken together, the AESOP's scales appear to be useful measures of selfobject needs that discriminate between personality functions and psychological outcomes as predicted by Kohut's original conceptualization. Indeed, the scales had good accuracy when discriminating persons who would fall above clinical criteria - more than 7 out of 10 cases were correctly classified across assessed outcomes. Of course, the utility of the present scales in clinical diagnosis and 
intervention remains to be further tested. Nonetheless, the measure's scale structure successfully passed tests of construct and predictive validity.

Yet perhaps a conceptually troubling aspect of the present scale was the frequency of correlations of symptom measure with the Mirroring selfobject need, as it was consistently strongly related to essentially all domains of psychopathology, thereby limiting its ability to discriminate between individuals. Though Mirroring is cited as the most common unmet selfobject need (e.g., Lee \& Martin, 1991), and its effects are theorized to be broad, the pattern of results might also reflect that it is tapping into a more general construct (e.g., negative emotionality).

Furthermore, although Kohut's theories are more generally applied to the covert form of narcissism, and two of the three selfobject needs were found to correlate with the overt form of narcissism, the two clusters did not differ significantly on scores on the measure of overt narcissism. The implications of this result are uncertain, as the present measure was nonetheless successful at discriminating between high-scores on the measure of covert narcissism, but it appears that the present measure may prove less useful in regards to the more traditional discussion of narcissistic pathology.

Viability of Self-Report

To measure the extent to which self-report bias might color the present results, the Paulhus Deception scales were included in the present battery. This instrument provides a measure of deceiving oneself, as well as deceiving others. Had the present measure produced a significant positive correlation with either scale, this would offer an important caution as to the meaningfulness of its relationship to other self-report measures. In the current analysis, no scale of the present measure demonstrated such a positive correlation, instead, Mirroring was found to 
negatively correlate with both scales, while Twinship was found to negatively correlate with selfdeception. These findings may reflect the fact that high scores on the present measure require a certain awareness of one's own needs as well as a willingness to report them, thus precluding a tendency to deny one's own emotional desires. This further offers support, at least in a circumspect sense, of the validity of the present results. Nonetheless, returning to the results of the cluster analysis, when examining the approximately $30 \%$ of the sample demonstrating relatively lower scores on the present measure, there was a significant increase in the tendency to engage in self-deception. Thus, the present measure appears vulnerable to the respondent's tendency towards denial, rendering low-scores on the measure difficult to interpret.

\section{Convergent and Discriminant Validity}

Attachment. Scores on the present measure's selfobject needs revealed a consistent pattern of relationships across the noted attachment styles. All three scales were positively correlated with the fearful and preoccupied attachment styles. Fearful attachment styles are defined as being high on attachment anxiety and attachment avoidance, while high attachment anxiety and low attachment avoidance is defined as a preoccupied attachment style. Both attachment styles are defined by a desire for close relationships with others, but concerns about one's ability to obtain them (either out of a concern of being hurt, or a concern of not being valued).

Some theorists have conceptualized selfobject needs as being part of a larger attachment motivational system (e.g.,Lichtenberg, 1988), offering selfobject needs as a kind of desire for affiliation. Given that selfobject needs are relational in nature, characterizing them as affiliative pursuits does not appear unduly controversial. In the present results, that an individual experiencing unmet selfobject needs would similarly experience anxiety regarding the possibility 
of meaningful connection with other individuals, is highly intuitive. The positive correlation with these characteristics is thus expected.

The need for Mirroring was the sole selfobject need to produce a deviation from the pattern of the other selfobject needs, as it was negatively correlated with healthy attachment and the dismissive attachment style (the other needs were non-significantly related). Dismissive attachment is defined as high avoidance of meaningful relationships, combined with a lack of anxiety. Individuals who report high mirroring needs may be described as appearing as disinterested in establishing meaningful relationships.

That Mirroring was the only selfobject need to be negatively correlated with both the healthy and dismissive forms of attachment is interesting, as it would not be unreasonable for all the selfobject needs to demonstrate such a relationship. One would expect that an individual with unmet selfobject needs would be less likely to develop a secure form of attachment, and one might further expect that the neediness conveyed by elevations on the current measure would stand counter to the self-reported disinterest found in the dismissive form of attachment.

Nonetheless, a unique element of the need for Mirroring is that it is the most dependent upon the responses of an outside party. Whereas Twinship and Idealizing require that an individual recognize a value within another person (either an essential relatedness or a trait worthy of admiration), Mirroring is more specifically oriented to receiving responsiveness from others. As mentioned previously, elements within each selfobject need can be found within the other needs, but Mirroring requires a unique kind of responsiveness on the part of other individuals. A person requiring Mirroring must be in a position to elicit such a response from a third party, whereas one could develop a sense of Twinship or Idealizing "from a distance." As such, individuals with this need may be the least likely to demonstrate the distance found within 
the dismissive attachment style. In regards to healthy attachment, as will be seen in later discussions, the need for Mirroring appears to be connected with the greatest amount of pathology, of which the negative correlation with secure attachment may represent another example. In some respects, the parental sensitivity required to provide mirroring experiences, may represent the most fundamental aspect of attachment, and its absence may be the most damaging (Gomez, 1997).

Narcissism. The distinction between the covert and overt forms of narcissism has already been discussed in great detail, and it was noted that the covert form of narcissism has consistently demonstrated a strong relationship with the fearful and preoccupied forms of attachment, the very pattern observed in the AESOP's attachment-related relationships. This continuity was also seen in the consistently strong correlations between the selfobject needs and the Hypersensitive Narcissism Scale. Although both Mirroring and Idealizing were found to correlate significantly with the measure of overt narcissism, a much stronger relationship was observed between the present measure's scales and the covert form of narcissism (both in terms of magnitude and the relationship being seen across all three selfobject needs).

This was an expected finding, as Kohut's theory of selfobject needs has been most often employed in the exploration of this covert form of narcissism. Indeed, much of Kohut's work was derived from perceived deficiencies in existing psychological theory to address the patients with covert narcissism that he was treating. Furthermore, the nature of overt narcissism may significantly reduce the endorsement of neediness as described by the present measure, as well as its accompanying vulnerability (Dickinson \& Pincus, 2003). Thus, although the developmental history of a person demonstrating overt narcissist is likely interspersed with unmet selfobject needs, the willingness to endorse such needs, and the specificity with which selfobject needs 
were developed to match the experience of the person with overt narcissist, is much less than that of the overt presentation.

Shame. As discussed in Chapter 5, the concept of shame has been identified as a core component of narcissism, and is defined as a perceived weakness within the whole self (Lewis, 1971). In the case of the overt narcissist, the clinical presentation of grandiosity and arrogance is seen as a defensive maneuver to maintain feelings of success and importance, thereby suppressing the experience of shame. In the case of covert narcissism, there is a strong desire to connect with the internal experience of grandiosity, but the ability to do so is hampered by a belief that the desire for this grandiosity is shameful. Kohut himself described shame as a disruption in the narcissitic equilibrium (Kohut, 1971), attributing the development of shame to the disapproval of parental figures. In essence, shame can be seen as a response when those looked to for selfobject responsiveness provide a sense of disinterest or negativity. Thus, the parental shortcomings that place an individual at risk for shame, are speculated to also give rise to unmet selfobject needs. The two should be positively correlated, in other words.

Precisely this relationship was found, as all three of the selfobject needs demonstrated a positive correlation with the measure of shame, again offering support of Kohut's contention that self-injury and shame are related constructs. By way of comparison, only two of the three selfobject needs were found to correlate with the experience of guilt, and in those two instances, the correlations were lower than their relationships with shame, an expected finding.

Clinical Symptoms. Kohut's view of psychopathology can be defined as relational in nature. According to Kohut, failure to receive the needed selfobject experiences results in an injured sense of self, such that future behavior deemed pathological represents efforts to protect the injured self, as well as an attempt to pursue the desired selfobject experiences. Conversely, 
self psychology describes the healthy self as allowing for the experience of positive self-regard and strength. Healthy individuals demonstrate a flexibility in meeting their selfobject needs, feel comfortable being empathically open to others (this comfort is due to a perceived lack of need for defensiveness), which in turn facilitates greater selfobject responsiveness from those in their environment, and have a positive relationship with their own emotional experiences (i.e., strong emotions are experienced as rejuvenating and necessary, as opposed to threatening; Lessem, 2005).

As such, disrupted interpersonal relationships, affective disturbances, and maladaptive behavioral patterns are theorized to represent an effort to protect and bolster a vulnerable self while maintaining the desired connections. Thus, self-esteem, personal agency, and selfcohesion are expected to fluctuate from low to high, and are therefore, offered as targets for intervention in a self psychology model, as self psychology emphasizes functional deficits of the self, as opposed to specific symptoms.

Because of this approach, it is theorized that numerous clinical symptoms may be attributable to deficits in self-structure. Accordingly, the multitude of positive correlations between the selfobject needs and the symptoms on the Brief Symptom Inventory is to be expected. Indeed, Mirroring was found to correlate with all of the symptoms, while Twinship was positively correlated with all but one of the Brief Symptom Inventory's 's scales (i.e., somatization). Conversely, Idealizing correlated only with obsessive-compulsive, interpersonal sensitivity, anxiety, and paranoid scales on the Brief Symptom Inventory.

It should first be noted that despite the fact that Twinship demonstrated the fewest number of correlations with the scales of the Brief Symptom Inventory, it nonetheless demonstrated a significant positive correlation with half of the measure's indices. Thus, as with 
the other selfobject needs, unmet need for Twinship appears to have numerous potential psychological consequences. However, Idealization's differences may be attributable to two primary discrepancies from the other selfobject needs. First, unmet Idealization may be expressed via sublimation, such as adopting serious convictions and beliefs (Silverstein, 2007). Furthermore, the perceived shortcomings in a need for Idealizing may be viewed as a failure on the part of other persons (e.g., a disappointing father), instead of being readily apparent as an issue with one's own status as depressed.

The positive correlation between the three scales of the present measure across multiple Brief Symptom Inventory indices offers support for the clinical nature of the purported deficits in selfobject needs. Furthermore, that these relationships were demonstrated across multiple clinical scales appears congruent with Kohut's model of psychopathology as reflecting structural deficits, as opposed to a specific link to one individual symptom.

It need be mentioned, however, that there is a distinction between distress and psychopathology. An important contribution of Kohut's theory was that narcissism was part of a normal developmental trajectory, and that the need for selfoject responsiveness was persistent throughout life. Healthy development was not characterized by a lack of need for selfobject responsiveness, but rather, a less intense experience of selfobject needs, as well as a greater flexibility in having the needs met. Thus, given the proper circumstances, any individual will experience distress resulting from unmet selfobject needs. The response to these unmet needs may result in notable impairment, but could also be expressed as a mild sadness that would fall far below a diagnosable condition. Thus, when investigating correlational patterns, one should be cautioned in recognizing that a positive correlation between the present measure and a scale 
on the Brief Symptoms Inventory does not necessarily imply the presence of a psychiatric diagnosis, or even a significant difficulty.

Other Selfobject Measures. All three of the selfobject need scales were negatively correlated with the Superiority scale of the Superiority and Goal Instability Scales (Robbins \& Patton, 1985). This scale is designed to measure Kohut's grandiose pole of the self. Kohut (1977) posited that when the selfobject needs of an individual went unmet or were disrupted in some way, the individual would later develop a compensatory mechanism to restore the desired internal functioning. These compensatory mechanisms represent a kind of second chance to restore a sense of self-cohesion. For example, an individual who presents as grandiose might be able to receive the need for mirroring that was previously missed. It may be conceived of a means to strengthen the self, standing in contrast to more traditional psychic defenses (Silverstein, 1999).

This grandiosity is displayed in items such as "I am witty and charming to others." Though Kohut did not intend these items to necessarily reflect a pathological stance (only after these compensatory strategies fail would more characterological issues take root), the disposition reflected in these comments stands in contrast to those endorsing a need for selfobject experiences. Indeed, it is unclear if endorsement of the items reflects an individual displaying a compensatory mechanism, or an overt form of narcissism. As such, a negative correlation between the two is to be expected.

More unexpectedly, the only selfobject need scale that did not demonstrate a significant positive correlation with the Goal Instability Scale was that of Idealizing, precisely the scale that would have theoretically expected the strongest correlation. This unexpected finding may in part be derived from the fact that the idealization in the Goal Instability scales are meant to tap a lack 
of direction in one's life, thus its emphasis concerns a connection to various academic outcomes, a limited aspect of the overall experience of lacking Idealization. Items such as "After a while, I lose sight of my goals," reflect this career focus. It might be the case that this specific aspect of lack of Idealization may not be entirely congruent with the broader clinical aspects tapped by the present measure.

The Social Connectedness scale is designed to measure an individual's sense of belongingness, and thus its negative correlation with the selfobject needs scales falls along expected lines. However, that Mirroring demonstrated the strongest negative correlation with the measures was surprising, particularly given that the need for Twinship is meant to tap the same construct. This may suggest an avenue for improvement on the Twinship domain, particularly in regards to themes of social connection. The mixed nature of selfobject experiences suggests that the correlation with Mirroring is not problematic, though the small magnitude of the correlation with Twinship could likely be improved.

SONI. An extensive exploration of the relationship between the AESOP and the SONI has already been provided. Most notably, the approach axes of the SONI demonstrated a consistently high correlation with the scales of the present measure. Furthermore, the strongest relationship in each case was found the corresponding selfobject need (e.g., the Mirroring scale of the present measure was most highly correlated with the approach mirroring scale of the SONI). The similar construction and intent of the measures makes this a highly understandable finding. Some of the findings were more contradictory, such as the Mirroring scale having a positive correlation with the avoidance of idealization and twinship. This somewhat curious finding may in part be explained by the confused factor structure of the SONI. Because of the significant cross-loadings across the measure's factors, the meaning behind its individual 
relationships is somewhat unclear. Nonetheless, the broad similarities between the approach axes and the present measure offer additional evidence for its validity.

Summary Remarks, Limitations, and Directions for Future Study

Evidence for the validity of the AESOP may be derived from the agreement provided by expert raters, initial statistical analyses suggesting a clean factor structure along the predicted three-factor lines, a confirmation of the proposed model with an independent sample, and the measure's consistent adherence to prediction relationships within the nomological network. In short, significant evidence for the measure's validity was provided.

Despite this positive assessment, there are important limitations to the present research. First, although independent samples were drawn to create and then confirm the measure's structure, the extent to which individuals participated in both studies is unknown. Furthermore, that both samples were drawn from the same university population suggests that there may be inherent limitations to the generalizability of the sample as a whole. Replication of these findings in different samples is thus required.

Second, the replication of these findings among clinical samples is critical. Given that the notion of selfobject needs, and a tool to measure said needs, are primarily of a clinical interest, demonstration of convergent and discriminant validity among a nonclinical sample is highly limited in providing evidence of the present measure's validity.

Third, even though the measure enjoys a relatively clean factor structure and demonstrated the expected relationships with other measures, this does not ensure that it is in fact measuring the intended construct. It is also unclear if it measures the intended construct in the most parsimonious or comprehensive way. Additional theoretical and statistical review of the measure might provide evidence of additional items that could be included in the measure to 
provide a better assessment of selfobject needs.

Fourth, the cross-sectional nature of the data makes its predictive implications somewhat limited. For example, although the self-psychology model would predict that unmet selfobject needs would lead to symptoms such as depression or anxiety, it might be the case that the causal pathway actually works in reverse. Similarly, numerous moderating and mediating relationships remain possible. Longitudinal and experimental methodologies will likely be required to address this shortcoming.

Finally, all of the present measures are self-report. The possibility of the results being driven by mono-method variance (i.e., sole reliance of self-report measures) cannot thus be discounted. Future research involving experimental conditions, combined with alternative measurement strategies (e.g., performance-based measures of personality) would provide an important next step. Additionally, testing the measure's predictive abilities in an experimental condition could provide an important test of its overall utility. Related to the second criticism, convergent validity from important clinical sources, such as therapist ratings of the client's transference, are also crucial. 


\section{CHAPTER XI}

\section{MAKING USE OF THE RESULTS}

In an ideal sense, the AESOP's worth would extend beyond its psychometric properties and convergent/discriminant validity, and would provide a source of clinical utility. As a demonstration of how results on the present measure might provide clinically relevant information, a composite hypothetical therapy client profile derived from several of the respondents from Study 2 will be reviewed. The composite nature of the profile will serve to protect the confidentiality of the respondents.

The individual in question is a 27 -year-old Caucasian woman, entering treatment citing concerns of depression and anxiety. In the initial assessment battery, consisting of the measures included in the present research, the individual demonstrates a clinical elevation on the Brief Symptom Inventory's anxiety and depression scales, a fearful attachment style on the Relationship Questionnaire, and a prominent elevation on the Idealizing scale (falling in the $84^{\text {th }}$ percentile in the present sample, as opposed to $61^{\text {st }}$ and $55^{\text {th }}$ percentile on the Mirroring and Twinship scales, respectively).

Based upon this initial profile, difficulties in anxiety and depression will likely serve as a motivation for entry into treatment, perhaps in conjunction with experiences of interpersonal dissatisfaction. The preoccupied form of attachment suggests that the client may have an interest in forming a strong relationship with the therapist, but may feel fearful about doing so. Given the expressed need for Idealization, some of this ambivalence likely derives from a profound sense of disappointment in primary caregivers. If this is indeed the case, initial investigations in therapy might focus on these early relationships, as well as current examples of idealized relationships. The client's previous experiences in therapy could provide a fruitful avenue to 
assess idealization, as the client might make comments such as "My old therapist understood me perfectly." The client might prove initially hesitant to engage in treatment, though this could suddenly change as the client experiences an unexpected gain from the therapy (Kohut would conceptualize this increase in vitality as derived from being in contact with an idealized figure i.e., the therapist). The therapist might experience this gain as startling, as it could take place prior to the therapist engaging in a meaningful interventions that he or she is aware.

If this is indeed the case, the therapist should be aware of numerous factors. For example, the client may experience absences as a source of great distress, reporting an increase in depression and anxiety in the case of a missed appointment or the therapist scheduling a break in the treatment (this distress would be derived from the absence of a newly idealized object). Similarly, such a client would likely be highly sensitive to perceived shortcoming on the part of therapist, becoming highly upset in the face of being misunderstood. Whereas one might initially experience these outbursts as a difficulty in anger management or hostility, these are perhaps more accurately understood as an injury to the self. Therapist shortcomings may be experienced as a repetition of the shortcomings of other previously idealized figures. A therapist unaware of these dynamics might be inclined to regard the anger as a defensive reaction or ignore the relational aspects of the exchange, thereby compounding the initial injury.

Such a profile carries implications for the therapist's disposition as well. If the client engages in an explicit form of idealization such as praising the therapist's skill or reporting an immediate decrease in symptoms, the therapist might feel uncomfortable or fraudulent. A therapist in this position might feel the need to dismiss the noted gains or praise, offer jokes regarding the quality of his/her work, or repeatedly seek to remove the praise through vigorous interpretation. Therapists who struggle to carry the weight of this idealization may respond to 
the discomfort produced by the idealization, perhaps leading to a potentially harmful or nonempathetic response. Conversely, a therapist who experiences criticism when the client feels as though the therapist has "failed," or who recognizes the client's idealization beginning to fade, may result in the therapist feeling insulted or rejected. The therapist's ability to successful navigate these experiences and interpret them correctly, may be in large part dependent upon the ability to understand the process taking place.

Of course, these comments are all speculative in nature, and no assessment can be reasonably interpreted in the absence of a thorough clinical interview. Furthermore, the process of therapy is interactive and personalized, thus it cannot be distilled to a formulaic approach such as "Need for Idealization necessitates unexpected anger towards the therapist will arise." Nonetheless, as the present discussion suggests, these needs may speak to a client's personal history, issues in transference and countertransference, and potentially difficulties in the treatment to navigate. 


\begin{tabular}{|c|c|c|c|c|c|c|}
\hline Item & $\begin{array}{l}\text { Average } \\
\text { Clarity }\end{array}$ & $\begin{array}{l}\text { Average } \\
\text { Relevance }\end{array}$ & $\begin{array}{r}\mathrm{Cl} \\
\text { Ratin }\end{array}$ & $\begin{array}{l}\text { arity } \\
\text { g Range }\end{array}$ & $\begin{array}{r}\text { Rel } \\
\text { Ratin }\end{array}$ & $\begin{array}{l}\text { evance } \\
\text { lg Range }\end{array}$ \\
\hline 1. I try to surround myself with successful people. & 4.00 & 4.00 & 3 & 5 & 3 & 5 \\
\hline 2. Sometimes I feel like I have a special connection with famous people. & 3.25 & 3.75 & 2 & 4 & 3 & 5 \\
\hline 3. I am attracted to highly ambitious people. & 3.75 & 3.75 & 2 & 5 & 3 & 4 \\
\hline $\begin{array}{l}\text { 4. I put a lot of value in the time I spend with talented people. } \\
\text { 5. There is at least one person in my life that I look to as a model of how I would }\end{array}$ & 3.25 & 3.50 & 3 & 4 & 3 & 4 \\
\hline like to be. & 3.75 & 3.50 & 3 & 5 & 3 & 5 \\
\hline 6. Whenever I can, I try to spend time with people that I look up to. & 4.00 & 4.00 & 4 & 4 & 4 & 4 \\
\hline 7. You should surround yourself with people that are impressive in some way. & 3.00 & 3.25 & 2 & 4 & 2 & 5 \\
\hline 8. When I need help, I feel better when there is someone there to manage things. & 3.00 & 3.00 & 2 & 4 & 2 & 4 \\
\hline 9. I like having someone I look up to provide me with advice. & 3.75 & 3.75 & 3 & 4 & 3 & 4 \\
\hline 10. I feel safe when I am around people who are calm and confident. & 4.25 & 3.50 & 3 & 5 & 3 & 5 \\
\hline 11. I don't need help from people with any expertise, I just need support.* & 2.25 & 2.50 & 2 & 3 & 2 & 4 \\
\hline $\begin{array}{l}\text { 12. It's important to have people that you admire in your life. } \\
\text { 13. It's important to me that I have a career that other people have a lot of }\end{array}$ & 4.00 & 4.00 & 3 & & 4 & 4 \\
\hline $\begin{array}{l}\text { respect for. } \\
\text { 14. People are sometimes jealous of the connections that I have or the people }\end{array}$ & 3.50 & 2.50 & 2 & & 2 & \\
\hline that I know. & 3.50 & 2.75 & 2 & 5 & 2 & 5 \\
\hline 15. I do very well in the company of powerful people. & 3.75 & 3.00 & 2 & 5 & 2 & 5 \\
\hline $\begin{array}{l}\text { 16. I enjoy watching movies where heroes "play by their own rules". } \\
\text { 17. Sometimes I feel like I have a special connection with artists/musicians when }\end{array}$ & 3.75 & 2.75 & 2 & & 2 & 3 \\
\hline I see/hear their work. & 2.75 & 2.75 & 1 & 4 & 1 & 4 \\
\hline $\begin{array}{l}\text { 18. I wish that I could have looked up to my parents more. } \\
\text { 19. The world is a scary place, and we need to be in contact with people who can }\end{array}$ & 4.00 & 3.25 & 2 & 5 & 2 & 5 \\
\hline $\begin{array}{l}\text { give us courage. } \\
20 \text {. I only support politicians and community leaders that are above all of the }\end{array}$ & 3.00 & 3.00 & 3 & 3 & 3 & 3 \\
\hline corruption that we see. & 2.50 & 2.75 & 2 & 3 & 2 & 4 \\
\hline 21. I tend to date people who are more competent than I am. & 2.75 & 2.50 & 2 & 4 & 2 & 3 \\
\hline 22. I feel better when I have someone to serve as my mentor. & 4.25 & 3.75 & 3 & 5 & 3 & 4 \\
\hline
\end{tabular}


23. When I'm away from the people that I think highly of, I feel empty inside.

24. I want to have people that I can turn to who will tell me what to do.

25. It hurts when people don't live up to my expectations.

26. I try hard to emulate people who I think highly of.

27. The best way to get ahead in life is to follow in the footsteps of the great people who have come before you.

28. I'm jealous of other people's connections.

29. I feel better when I'm in the presence of wise people.

30. A person's greatest strength is the people they know.

31. It's important to me that I associate with groups or clubs that are well thought of by others.

32. I can recall an occasion when I was ashamed of one of my parents.

33. One of the worst things that loved ones can do is be unreliable.

34. I feel fragile when I don't have strong people in my life to lean upon.

35. I sometimes dream about meeting famous and influential people.

36. I like to think of myself as "My own person".*

37. I dislike the thought of being greatly influenced by other people.*

38. I get very angry when people disappoint me.

39. I sometimes take comfort in the knowledge that there is a higher power that I can connect with.*

40. I want to be close to people who have something unique and special about them.

41. I have a lot of weaknesses, but with help from the right people, I could accomplish a lot.

42. I tend to put people on a pedestal.

43. It's devastating when I'm disappointed by the people I respect in my life.

44. When I'm stressed, I think of how a hero would react.

45. I feel like I'm meant to be part of something greater. 
47. It hurts me when I see the people I respect not living up to their potential.

48. I get highly invested in the trials and tribulations of celebrities.

49. I am good at calming myself down.*

50. I tend to have faith in authority figures. 


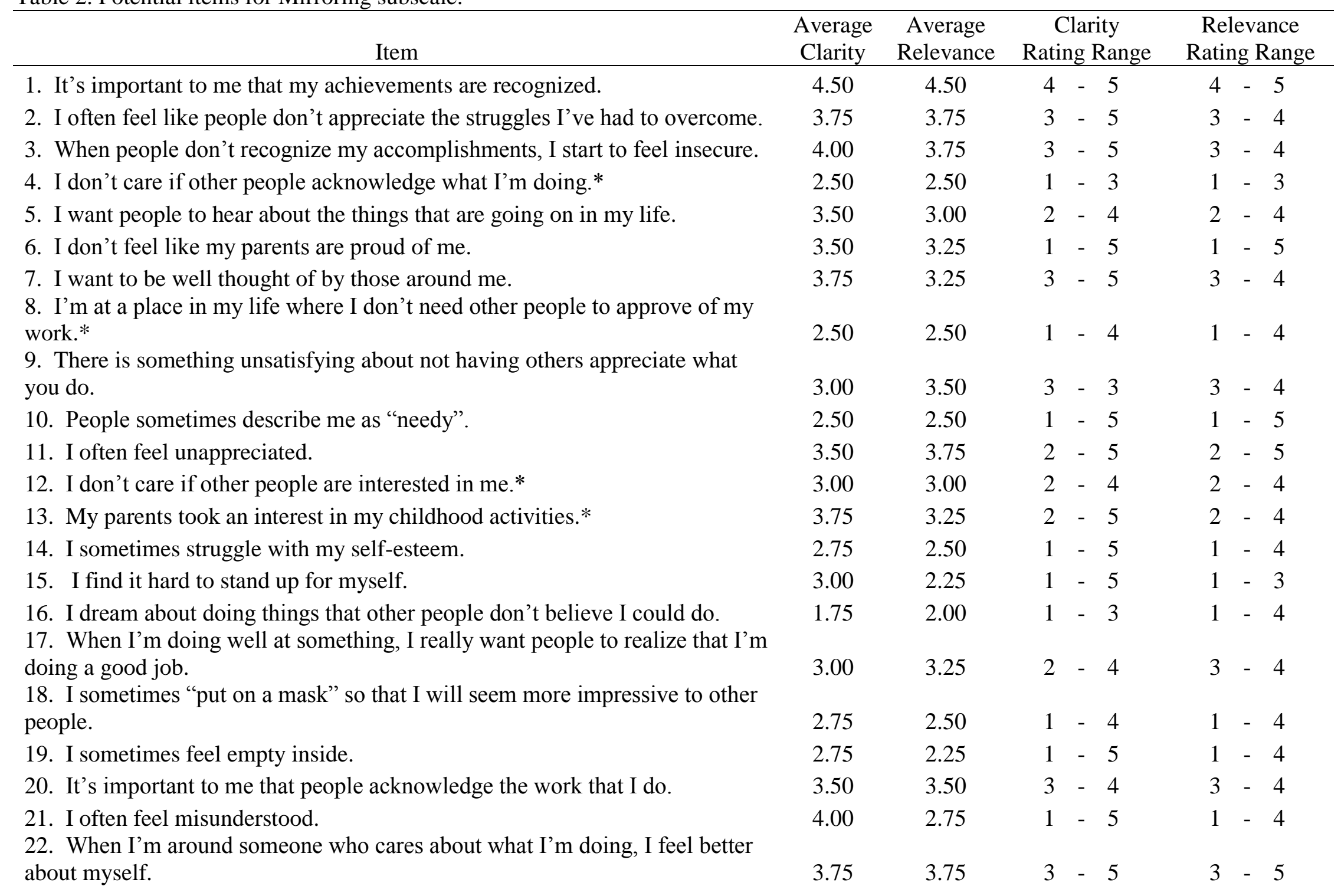


23. I'm devastated when people criticize me.

24. I've been known to "snap" at people from time to time.

25. Life would be a lot better if people appreciated me.

26. I sometimes struggle with feelings of worthlessness.

27. The most important thing in a relationship is that your partner truly listens to you.

28. I wish people understood me better.

29. My parents criticized me a lot when I was younger.

30. When things go wrong I have a tendency to fall apart.

31. If people don't care about what I'm doing, I find it hard to keep going.

32. My excitement is rarely shared by others.

33. I don't feel as though I have many talents.

34. I crave the admiration of other people.

35. I keep a lot of my hopes and dreams to myself.

36. I do well in the face of adversity.*

37. I feel as though the people in my life haven't celebrated my accomplishments.

38. I wish the people close to me cared more about my feelings..

39. Sometimes I feel hollow, like something inside of me is missing.

40. I feel as though I'm often overlooked.

41. People would describe me as energetic.*

42. I fantasize about being recognized for the work that I do.

43. I prefer to go unnoticed.*

\begin{tabular}{|c|c|c|c|c|c|c|}
\hline 3.75 & 3.25 & 1 & -5 & 1 & - & 5 \\
\hline 3.00 & 1.25 & 1 & -5 & 1 & - & 2 \\
\hline 3.50 & 3.25 & 2 & -4 & 2 & - & 5 \\
\hline 3.75 & 3.00 & 1 & -5 & 1 & - & 5 \\
\hline 3.25 & 3.25 & 2 & -4 & 2 & - & 5 \\
\hline 3.25 & 3.25 & 2 & -4 & 2 & - & 5 \\
\hline 2.75 & 2.75 & 1 & -4 & 1 & - & 5 \\
\hline 2.75 & 2.50 & 1 & -4 & 1 & - & 4 \\
\hline 3.25 & 3.50 & 2 & -4 & 2 & - & 4 \\
\hline 2.50 & 2.50 & 1 & -5 & 1 & - & 4 \\
\hline 3.00 & 1.75 & 1 & -4 & 1 & - & 3 \\
\hline 4.00 & 4.00 & 3 & -5 & 3 & - & 5 \\
\hline 2.75 & 2.75 & 1 & -4 & 2 & - & 4 \\
\hline 2.75 & 1.75 & 1 & -4 & 1 & - & 3 \\
\hline 3.50 & 3.75 & 3 & 4 & 3 & - & 5 \\
\hline 3.00 & 2.25 & 1 & 4 & 1 & - & 3 \\
\hline 2.25 & 3.25 & 1 & 4 & 1 & - & 5 \\
\hline 3.25 & 2.50 & 1 & -5 & 1 & - & 4 \\
\hline 2.75 & 1.75 & 1 & -4 & 1 & - & 3 \\
\hline 3.75 & 4.00 & 3 & -5 & 3 & - & 5 \\
\hline 3.75 & 3.50 & 2 & 5 & 2 & & 5 \\
\hline
\end{tabular}




\begin{tabular}{|c|c|c|c|c|c|c|}
\hline Item & $\begin{array}{l}\text { Average } \\
\text { Clarity }\end{array}$ & $\begin{array}{l}\text { Average } \\
\text { Relevance }\end{array}$ & & $\begin{array}{l}\text { Clarity } \\
\text { Rating Range }\end{array}$ & \multicolumn{2}{|c|}{$\begin{array}{r}\text { Relevan } \\
\text { Rating Ra }\end{array}$} \\
\hline $\begin{array}{l}\text { 1. It helps a lot to be around people going through the same things I'm going } \\
\text { through. }\end{array}$ & 3.50 & 3.50 & 3 & -4 & 3 & - \\
\hline 2. I try to stay away from people who are in similar situations as me.* & 2.50 & 2.75 & 1 & 4 & 2 & - \\
\hline 3. I like talking with people when we have similar opinions. & 3.75 & 3.25 & 3 & 5 & 3 & - \\
\hline 4. The people I associate with should share my lifestyle. & 2.50 & 2.75 & 2 & 3 & 2 & - \\
\hline 5. I want people close to me to share my current circumstances. & 2.50 & 3.00 & 1 & 3 & 3 & - \\
\hline 6. I try to find friends whose personalities contrast with mine.* & 2.75 & 2.75 & 1 & 5 & 2 & - \\
\hline 7. I like knowing that my feelings are shared by others. & 3.75 & 3.50 & 3 & 5 & 3 & - \\
\hline 8. I like to belong to groups of people whose personalities are similar to mine. & 3.25 & 2.25 & 1 & 4 & 1 & - \\
\hline 9. It's boring if the people around me share most of my thoughts and opinions.* & 2.25 & 3.00 & 1 & 3 & 2 & - \\
\hline $\begin{array}{l}\text { 10. It's easier for me to handle stress when I know that other people are going } \\
\text { through the same thing. }\end{array}$ & 3.50 & 3.75 & 3 & -4 & 3 & - \\
\hline 11. I want my friends to share my beliefs. & 4.00 & 4.00 & 3 & 5 & 3 & - \\
\hline 12. I always wanted a best friend who is just like me. & 4.25 & 4.00 & 4 & 5 & 4 & - \\
\hline 13. I like being part of a team or a group. & 4.00 & 3.00 & 2 & 5 & 2 & - \\
\hline 14. I used to imitate my parents' behavior when I was a child. & 3.25 & 2.25 & 1 & 4 & 1 & - \\
\hline 15. Sometimes I worry that there is no one really like me. & 3.50 & 3.00 & 2 & 4 & 2 & - \\
\hline 16. I have a strong need to belong. & 3.25 & 2.25 & 1 & 5 & 1 & - \\
\hline 17. I try to define myself by being unique.* & 3.00 & 2.25 & 1 & 5 & 2 & - \\
\hline 18. I have a lot of loyalty to a particular group. & 3.00 & 2.75 & 2 & 3 & 2 & - \\
\hline 19. I dress similarly to a lot of my friends. & 3.25 & 3.00 & 2 & 4 & 2 & - \\
\hline 20. I try to stand out through my taste in music or movies.* & 2.75 & 2.25 & 1 & 5 & 1 & - \\
\hline 21. I struggle with feelings of loneliness. & 3.25 & 2.50 & 1 & 5 & 1 & - \\
\hline 22. Sometimes it helps to be around someone, even if we aren't talking. & 3.50 & 3.00 & 2 & 5 & 2 & - \\
\hline 23. I hate being alone. & 3.25 & 2.50 & 1 & 5 & 1 & - \\
\hline 24. I define myself based on the people I spend time with. & 3.25 & 2.75 & 2 & -4 & 2 & - \\
\hline
\end{tabular}


25. I feel as though I'm an important part of a community.*

26. I have a hard time connecting with other people.

27. I sometimes feel as though there is something blocking me from getting close to other people.

28. I wish I had a group of friends that I felt really comfortable with.

29. I sometimes feel like I'm unusual in some way.

30. If I'm not doing things with my friends, I feel like I lose touch with them.

31. Being around people who are similar to me keeps me feeling grounded.

32. I'm happiest when I feel like I'm part of a larger community.

33. It's hard for me to connect with people who are different than I am.

34. Being around people who think like I do makes it easier to overcome difficulties or solve problems.

35. I am definitely not a "lone wolf".

36. Sometimes I feel as if I don't belong.

37. I find it hard to relate to people.

38. I like to stand out from my friends and family.*

39. I wish I could have more time away from everyone else.*

40. When I was very young, I always wanted to help my parents with their chores.

41. I want to be around people who share my values.

42. I find a lot of my close friends grow apart from me.

\begin{tabular}{|c|c|c|c|c|c|c|}
\hline 3.00 & 2.25 & 1 & -3 & 1 & - & \\
\hline 3.00 & 2.25 & 1 & -3 & 1 & - & 3 \\
\hline 3.25 & 2.00 & 1 & -5 & 1 & - & 4 \\
\hline 3.25 & 2.50 & 2 & -4 & 2 & - & 3 \\
\hline 2.75 & 2.50 & 2 & -3 & 2 & - & 4 \\
\hline 3.25 & 2.50 & 1 & -5 & 1 & - & 5 \\
\hline 3.50 & 3.50 & 3 & -4 & 3 & - & 4 \\
\hline 3.50 & 3.00 & 2 & -5 & 2 & - & 4 \\
\hline 3.75 & 3.25 & 2 & -5 & 2 & - & 4 \\
\hline 3.00 & 3.25 & 3 & -3 & 3 & - & 4 \\
\hline 3.25 & 2.25 & 2 & -4 & 2 & - & 3 \\
\hline 3.00 & 2.50 & 2 & -4 & 2 & - & 3 \\
\hline 3.00 & 1.50 & 1 & - 4 & 1 & - & 2 \\
\hline 2.75 & 2.00 & 1 & - & 1 & - & 3 \\
\hline 3.00 & 2.50 & 2 & -4 & 2 & - & 4 \\
\hline 3.00 & 2.00 & 1 & -5 & 1 & - & 4 \\
\hline 3.75 & 3.25 & 2 & -5 & 2 & - & 4 \\
\hline 3.00 & 2.25 & 1 & -4 & 1 & - & 4 \\
\hline
\end{tabular}


Table 4. Rotated factor solution of remaining AESOP items.

\begin{tabular}{|c|c|c|c|c|c|c|}
\hline & \multicolumn{3}{|c|}{ Pattern } & \multicolumn{3}{|c|}{ Structure } \\
\hline Item & Factor 1 & Factor 2 & Factor 3 & Factor 1 & Factor 2 & Factor 3 \\
\hline Idealizing 1 & & .496 & & & .486 & \\
\hline Idealizing 3 & & .545 & & & .571 & \\
\hline Idealizing 6 & & .655 & & & .680 & \\
\hline Idealizing 7 & & .422 & & & .473 & \\
\hline Idealizing 12 & & .614 & & & .651 & \\
\hline Idealizing 13 & & .341 & & & .398 & \\
\hline Idealizing 17 & & .588 & & & .612 & \\
\hline Idealizing 19 & & .349 & & & .372 & \\
\hline Idealizing 20 & & .581 & & & .597 & \\
\hline Idealizing 23 & & .376 & & & .406 & \\
\hline Mirroring 2 & .643 & & & .652 & & \\
\hline Mirroring 3 & .686 & & & .706 & & \\
\hline Mirroring 7 & .692 & & & .682 & & \\
\hline Mirroring 9 & .533 & & & .573 & & \\
\hline Mirroring 11 & .510 & & & .542 & & \\
\hline Mirroring 12 & .665 & & & .648 & & \\
\hline Mirroring 13 & .770 & & & .768 & & \\
\hline Mirroring 14 & .720 & & & .746 & & \\
\hline Mirroring 15 & .748 & & & .774 & & \\
\hline Mirroring 18 & .562 & & & .598 & & \\
\hline Mirroring 20 & .575 & & & .610 & & \\
\hline
\end{tabular}




\begin{tabular}{|c|c|c|}
\hline Mirroring 21 & .714 & .703 \\
\hline Mirroring 24 & .667 & .700 \\
\hline Twinship 1 & .627 & .711 \\
\hline Twinship 2 & .594 & .612 \\
\hline Twinship 3 & .565 & .589 \\
\hline Twinship 4 & .646 & .717 \\
\hline Twinship 5 & .422 & .429 \\
\hline Twinship 6 & .360 & .363 \\
\hline Twinship 8 & .570 & .627 \\
\hline Twinship 17 & .581 & .599 \\
\hline
\end{tabular}

Note. Loadings of under .32 are not indicated. 
Table 5. Reliability Statistics by Selfobject Need

\begin{tabular}{|c|c|c|}
\hline Item & Item-Total Correlation & $\begin{array}{l}\text { Cronbach's Alpha if } \\
\text { Item Were Deleted }\end{array}$ \\
\hline Idealizing 1 & .40 & .78 \\
\hline Idealizing 3 & .47 & .77 \\
\hline Idealizing 6 & .57 & .76 \\
\hline Idealizing 7 & .46 & .77 \\
\hline Idealizing 12 & .55 & .76 \\
\hline Idealizing13 & .40 & .78 \\
\hline Idealizing 17 & .51 & .76 \\
\hline Idealizing 19 & .40 & .78 \\
\hline Idealizing 20 & .53 & .76 \\
\hline Idealizing 23 & .41 & .78 \\
\hline Mirroring 2 & .62 & .91 \\
\hline Mirroring 3 & .69 & .91 \\
\hline Mirroring 7 & .63 & .91 \\
\hline Mirroring 9 & .57 & .91 \\
\hline Mirroring 11 & .52 & .91 \\
\hline Mirroring 12 & .59 & .91 \\
\hline Mirroring 13 & .72 & .90 \\
\hline Mirroring 14 & .71 & .90 \\
\hline Mirroring 15 & .73 & .90 \\
\hline Mirroring 18 & .60 & .91 \\
\hline
\end{tabular}




\begin{tabular}{lll}
\hline Mirroring 20 & .59 & .91 \\
\hline Mirroring 21 & .66 & .91 \\
\hline Mirroring 24 & .68 & .91 \\
\hline Twinship 1 & .58 & .77 \\
\hline Twinship 2 & .57 & .77 \\
\hline Twinship 3 & .53 & .78 \\
\hline Twinship 4 & .60 & .77 \\
\hline Twinship 5 & .43 & .79 \\
\hline Twinship 6 & .41 & .81 \\
\hline Twinship 8 & .51 & .78 \\
\hline Twinship 17 & .60 & .77 \\
\hline
\end{tabular}


Table 6. Confirmatory factor analysis measurement loadings.

\begin{tabular}{lrrr}
\hline Factor \& Item & Standardized & \multicolumn{2}{c}{ Unstandardized } \\
\hline Mirror & & & \\
14 & 0.79 & 1.00 \\
15 & 0.75 & 0.96 \\
3 & 0.75 & 0.95 \\
18 & 0.73 & 0.93 \\
24 & 0.73 & 0.90 \\
13 & 0.70 & 0.92 \\
7 & 0.68 & 0.86 \\
21 & 0.66 & 0.82 \\
2 & 0.64 & 0.80 \\
9 & 0.63 & 0.76 \\
12 & 0.60 & 0.73 \\
11 & 0.57 & 0.71 \\
20 & 0.53 & 0.74
\end{tabular}

Ideal

$\begin{array}{rll}20 & 0.60 & 1.00 \\ 17 & 0.54 & 0.82 \\ 6 & 0.53 & 0.83 \\ 12 & 0.53 & 0.75 \\ 13 & 0.50 & 0.94 \\ 23 & 0.45 & 0.76 \\ 7 & 0.41 & 0.75 \\ 19 & 0.40 & 0.86 \\ 3 & 0.34 & 0.59 \\ 1 & 0.31 & 0.46\end{array}$

Twin

\begin{tabular}{rll}
1 & 0.76 & 0.91 \\
4 & 0.75 & 1.00 \\
2 & 0.65 & 0.70 \\
3 & 0.65 & 0.74 \\
8 & 0.65 & 0.84 \\
17 & 0.53 & 0.63 \\
5 & 0.50 & 0.65 \\
6 & 0.39 & 0.58 \\
\hline
\end{tabular}

Note: All item loadings were significant $\mathrm{p}<0.001$. Unstandardized loadings equal to 1.0 were fixed for latent construct identification. 
Table 7. Summary of discriminant analysis

\begin{tabular}{lcccc}
\hline & \multicolumn{4}{c}{ AESOP } \\
\cline { 2 - 5 } & $\begin{array}{c}\text { Discriminant } \\
\text { Accuracy }\end{array}$ & $\begin{array}{c}\text { Wilk's } \\
\text { Lambda }\end{array}$ & $\begin{array}{c}\text { Cannonical } \\
\text { Correlation }\end{array}$ & $\mathrm{R}^{2}$ \\
\hline Covert Narcissism (HSNS) & 78.6 & 0.79 & 0.46 & 0.21 \\
Interpersonal Sensitivity (BSI) & 70.2 & 0.79 & 0.46 & 0.21 \\
Global Severity (BSI) & 71.4 & 0.79 & 0.46 & 0.21 \\
Self-Esteem (RSES) & 79.3 & 0.80 & 0.45 & 0.20 \\
Paranoid Ideation (BSI) & 70.8 & 0.80 & 0.45 & 0.20 \\
Social Connectedness (SCS) & 76.2 & 0.81 & 0.44 & 0.19 \\
Psychoticism (BSI) & 71.0 & 0.81 & 0.44 & 0.19 \\
Depression (BSI) & 69.5 & 0.81 & 0.43 & 0.18 \\
Idealization (SGIS) & 75.9 & 0.83 & 0.41 & 0.17 \\
Anxiety (BSI) & 69.8 & 0.84 & 0.41 & 0.17 \\
Obsessive-Compulsive (BSI) & 69.5 & 0.86 & 0.38 & 0.14 \\
Phobic Anxiety (BSI) & 67.6 & 0.88 & 0.35 & 0.12 \\
Hostility (BSI) & 67.1 & 0.89 & 0.34 & 0.12 \\
Shame (PFQ2) & 77.2 & 0.89 & 0.33 & 0.11 \\
Somatization (BSI) & 70.2 & 0.89 & 0.33 & 0.11 \\
Guilt (PFQ2) & 74.9 & 0.91 & 0.30 & 0.09 \\
Grandiosity (SGIS) & 72.0 & 0.93 & 0.27 & 0.07 \\
Narcissism (NPI) & 74.4 & 0.98 & 0.13 & 0.02 \\
\hline Note: All Wilk's lambda are significant & & & &
\end{tabular}

Note: All Wilk's lambda are significant, $\mathrm{p}<0.01$. 
Table 8. Summary of discriminant analysis: differential effects by sub-scale.

\begin{tabular}{lccc}
\hline & \multicolumn{3}{c}{ AESOP } \\
\cline { 2 - 4 } & & & \\
& MIRROR & IDEAL & TWIN \\
\hline Cover Narcissism (HSNS) & + & + & + \\
Global Severity Index (BSI) & + & & + \\
Interpersonal Sensitivity (BSI) & + & & + \\
Self-Esteem (RSES) & + & & \\
Paranoid Ideation (BSI) & + & & + \\
Social Connectedness (SCS) & - & & \\
Psychoticism (BSI) & + & + & + \\
Depression (BSI) & + & & + \\
Idealization (SGIS) & - & & + \\
Anxiety (BSI) & + & & + \\
Obsessive-Compulsive (BSI) & + & & \\
Phobic Anxiety (BSI) & + & & \\
Hostility (BSI) & + & & \\
Shame (PFQ2) & + & & - \\
Somatization (BSI) & + & & \\
Guilt (PFQ2) & + & & \\
Grandiosity (SGIS) & - & & \\
Over Narcissism (NPI) & & & + \\
\hline Not Only significant predictors & $<0.05)$ are shown; signs
\end{tabular}

Note: Only significant predictors $(p<0.05)$ are shown; signs correspond to the direction of effects in relationship to the clinical group membership. 
Table 9. Rotated factor loadings (pattern matrix) of SONI items.

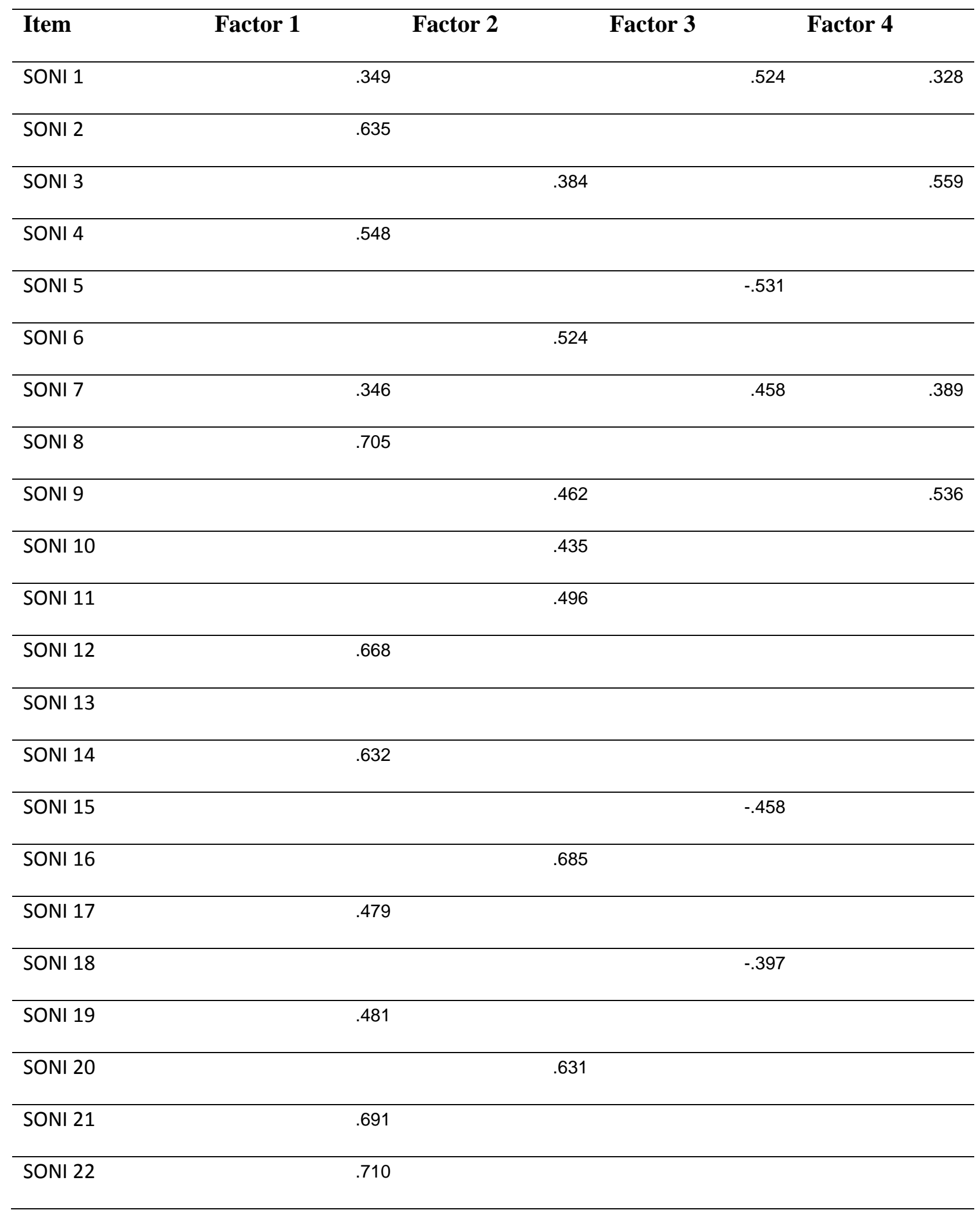




\begin{tabular}{|c|c|c|c|c|}
\hline SONI 23 & & & -.521 & \\
\hline SONI 24 & & & -.433 & \\
\hline SONI 25 & & .646 & & \\
\hline SONI 26 & .590 & & & \\
\hline SONI 27 & .697 & & & \\
\hline SONI 28 & & .680 & & \\
\hline SONI 29 & .364 & .357 & .439 & \\
\hline SONI 30 & & .370 & & .519 \\
\hline SONI 31 & .619 & & & \\
\hline SONI 32 & .686 & & & \\
\hline SONI 33 & .414 & & .372 & \\
\hline SONI 34 & & .536 & & .318 \\
\hline SONI 35 & & .387 & .341 & \\
\hline SONI 36 & & .434 & & \\
\hline SONI 37 & & .32 & -.366 & \\
\hline SONI 38 & & .544 & & \\
\hline
\end{tabular}

Note. Loadings of under .32 are not indicated. 
Table 10. Correlations amongst all assessed measures.

\begin{tabular}{|c|c|c|c|c|c|c|c|c|c|c|c|c|}
\hline & & 1 & 2 & 3 & 4 & 5 & 6 & 7 & 8 & 9 & 10 & 11 \\
\hline 1 & $\begin{array}{l}\text { Secure } \\
\text { Attachment } \\
(\mathrm{RQ})\end{array}$ & --- & $-.48^{* *}$ & $-.18^{* *}$ & $-.17^{\star *}$ & .05 & .06 & .04 & $.10^{* *}$ & $-.13^{* *}$ & .06 & -.02 \\
\hline 2 & $\begin{array}{l}\text { Fearful } \\
\text { Attachment } \\
(\mathrm{RQ})\end{array}$ & $-.48^{* *}$ & --- & $.23^{* *}$ & $.11^{* *}$ & -.06 & $-.10^{* *}$ & $-.08^{*}$ & $.08^{*}$ & $.15^{\star *}$ & $.10^{\star *}$ & $.17^{* \star}$ \\
\hline 3 & $\begin{array}{l}\text { Preoccupied } \\
\text { Attachment } \\
\text { (RQ) }\end{array}$ & $-.18^{\star \star}$ & $.23^{* \star}$ & --- & $-.13^{* *}$ & .02 & $-.20^{* *}$ & $-.16^{* *}$ & $.14^{\star *}$ & $.25^{\star *}$ & $.15^{\star \star}$ & $.36^{\star *}$ \\
\hline 4 & $\begin{array}{l}\text { Dismissive } \\
\text { Attachment } \\
\text { (RQ) }\end{array}$ & $-.17^{* *}$ & $.11^{* *}$ & $-.13^{* *}$ & --- & $.09^{*}$ & $.18^{* *}$ & .04 & -.04 & .06 & .00 & $-.13^{* *}$ \\
\hline 5 & $\begin{array}{l}\text { Narcissism } \\
\text { (NPI) }\end{array}$ & .05 & -.06 & .02 & $.09^{*}$ & --- & $.17^{* *}$ & $-.16^{* *}$ & $.08^{*}$ & $.13^{\star *}$ & $.28^{\star *}$ & $.15^{* \star}$ \\
\hline 6 & $\begin{array}{l}\text { Self- } \\
\text { Deception } \\
\text { (PDS) }\end{array}$ & .06 & $-.10^{* *}$ & $-.20^{* *}$ & $.18^{* *}$ & $.17^{* *}$ & --- & $.34^{* *}$ & $-.10^{* *}$ & $-.43^{* *}$ & $-.10^{\star \star}$ & $-.42^{* *}$ \\
\hline 7 & $\begin{array}{l}\text { Impression } \\
\text { Management } \\
\text { (PDS) }\end{array}$ & .04 & $-.08^{*}$ & $-.16^{* *}$ & .04 & $-.16^{\star *}$ & $.34^{\star *}$ & --- & -.05 & $-.28^{* *}$ & -.07 & $-.27^{\star *}$ \\
\hline 8 & $\begin{array}{l}\text { Approach- } \\
\text { Twinship } \\
\text { (SONI) }\end{array}$ & $.10^{* *}$ & $.08^{*}$ & $.14^{* *}$ & -.04 & $.08^{*}$ & $-.10^{* *}$ & -.05 & --- & $.08^{*}$ & $.61^{* *}$ & $.47^{* *}$ \\
\hline $\begin{array}{l}9 \\
10\end{array}$ & $\begin{array}{l}\text { Avoid- } \\
\text { Idealization } \\
\text { and Twinship } \\
\text { (SONI) } \\
\text { Approach } \\
\text { Idealization } \\
\text { (SONI) }\end{array}$ & $-.13^{\star *}$ & $.15^{\star *}$ & $.25^{* *}$ & .00 & $.28^{* *}$ & $-.43^{* *}$ & $-.28^{* *}$ & $.61^{* *}$ & $.21^{* *}$ & $.21^{* *}$ & $.48^{* *}$ \\
\hline
\end{tabular}




\begin{tabular}{|c|c|c|c|c|c|c|c|c|c|c|c|c|}
\hline & & 1 & 2 & 3 & 4 & 5 & 6 & 7 & 8 & 9 & 10 & 11 \\
\hline 11 & $\begin{array}{l}\text { Approach } \\
\text { Mirroring } \\
\text { (SONI) }\end{array}$ & -.02 & $.17^{* *}$ & $.36^{* *}$ & $-.13^{* *}$ & $.15^{* *}$ & $-.42^{* *}$ & $-.27^{* *}$ & $.47^{* *}$ & $.48^{* *}$ & $.48^{\star *}$ & --- \\
\hline 12 & $\begin{array}{l}\text { Avoid Mirroring } \\
\text { (SONI) }\end{array}$ & .04 & .00 & $-.08^{*}$ & $.19^{* *}$ & .02 & $.16^{\star *}$ & .06 & -.02 & $.14^{* *}$ & .02 & $-.24^{* *}$ \\
\hline 13 & $\begin{array}{l}\text { Covert } \\
\text { Narcissism } \\
\text { (HSNS) }\end{array}$ & $-.19^{* *}$ & $.27^{* *}$ & $.34^{* *}$ & .00 & .06 & $-.31^{* *}$ & $-.24^{* *}$ & $.32^{* \star}$ & $.47^{* *}$ & $.35^{\star *}$ & $.58^{* *}$ \\
\hline 14 & $\begin{array}{l}\text { Self-Esteem } \\
\text { (RSES) }\end{array}$ & $-.23^{* *}$ & $.25^{* *}$ & $.33^{\star *}$ & $-.14^{* *}$ & $-.26^{* *}$ & $-.49^{* *}$ & $-.23^{* *}$ & .02 & $.39^{* *}$ & .02 & $.44^{* \star}$ \\
\hline 15 & Shame (PFQ2) & $-.11^{* *}$ & $.25^{* *}$ & $.28^{* *}$ & -.06 & $-.17^{* *}$ & $-.32^{* *}$ & $-.25^{* *}$ & $.13^{* *}$ & $.32^{* *}$ & $.13^{* *}$ & $.39^{* *}$ \\
\hline 16 & Guilt (PFQ2) & $-.11^{* *}$ & $.22^{* *}$ & $.25^{* *}$ & -.03 & $-.10^{* *}$ & $-.34^{* *}$ & $-.20^{* *}$ & .04 & $.31^{* *}$ & $.10^{* *}$ & $.28^{* *}$ \\
\hline 17 & $\begin{array}{l}\text { Idealization } \\
\text { (SGIS ) }\end{array}$ & $.18^{* *}$ & $-.18^{* *}$ & $-.31^{* *}$ & .05 & $-.10^{* *}$ & $.40^{* *}$ & $.30^{* *}$ & -.05 & $-.33^{* *}$ & -.06 & $-.40^{* *}$ \\
\hline 18 & $\begin{array}{l}\text { Grandiosity } \\
\text { (SGIS ) }\end{array}$ & -.03 & .04 & $-.08^{*}$ & -.05 & $-.58^{* *}$ & -.03 & $.14^{\star *}$ & $-.19^{* *}$ & -.07 & $-.31^{* *}$ & $-.24^{* \star}$ \\
\hline 19 & $\begin{array}{l}\text { Social } \\
\text { Conenctedness } \\
\text { (SCS) }\end{array}$ & $.43^{* *}$ & $-.34^{* *}$ & $-.33^{* *}$ & -.02 & $.17^{* *}$ & $.34^{* *}$ & $.19^{* *}$ & $.08^{*}$ & $-.41^{* *}$ & .02 & $-.32^{* *}$ \\
\hline 20 & $\begin{array}{l}\text { Somatization } \\
\text { (BSI) }\end{array}$ & $-.17^{* *}$ & $.16^{* *}$ & $.23^{* *}$ & .01 & .03 & $-.30^{* *}$ & $-.20^{* *}$ & .00 & $.39^{* *}$ & $.10^{*}$ & $.32^{* *}$ \\
\hline 21 & $\begin{array}{l}\text { Obsessive- } \\
\text { Compulsize } \\
\text { (BSI) }\end{array}$ & $-.18^{* *}$ & $.29^{* *}$ & $.30^{* *}$ & -.01 & $-.10^{* *}$ & $-.34^{* *}$ & $-.18^{* *}$ & $.14^{* *}$ & $.30^{* *}$ & $.15^{* *}$ & $.40^{* *}$ \\
\hline
\end{tabular}




\begin{tabular}{|c|c|c|c|c|c|c|c|c|c|c|c|c|}
\hline & & 1 & 2 & 3 & 4 & 5 & 6 & 7 & 8 & 9 & 10 & 11 \\
\hline 22 & $\begin{array}{l}\text { Interpersonal } \\
\text { Sensitivty (BSI) }\end{array}$ & $-.22^{\star \star}$ & $.26^{* *}$ & $.38^{* *}$ & $-.09^{*}$ & $-.12^{\star \star}$ & $-.35^{\star *}$ & $-.19^{\star *}$ & $.15^{\star *}$ & $.38^{* *}$ & $.12^{* *}$ & $.47^{* *}$ \\
\hline 23 & $\begin{array}{l}\text { Depression } \\
\text { (BSI) }\end{array}$ & $-.24^{* *}$ & $.28^{* *}$ & $.34^{* *}$ & -.04 & $-.09^{*}$ & $-.31^{* *}$ & $-.23^{\star *}$ & .07 & $.40^{* *}$ & $.08^{*}$ & $.44^{* *}$ \\
\hline 24 & Anxiety (BSI) & $-.18^{\star *}$ & $.22^{* *}$ & $.29^{\star *}$ & -.02 & -.02 & $-.34^{\star *}$ & $-.22^{\star \star}$ & $.07^{*}$ & $.42^{\star \star}$ & $.14^{\star *}$ & $.40^{* *}$ \\
\hline 25 & Hostility (BSI) & $-.24^{* *}$ & $.23^{* *}$ & $.26^{* *}$ & .04 & .05 & $-.28^{\star *}$ & $-.26^{\star \star}$ & $.08^{*}$ & $.40^{\star *}$ & $.12^{* *}$ & $.34^{* *}$ \\
\hline 26 & $\begin{array}{l}\text { Paranoid } \\
\text { Ideation (BSI) }\end{array}$ & $-.30^{* *}$ & $.32^{* *}$ & $.32^{\star *}$ & .01 & $.07^{*}$ & $-.25^{\star *}$ & $-.20^{\star *}$ & $.14^{* *}$ & $.37^{\star *}$ & $.16^{* *}$ & $.44^{* *}$ \\
\hline 27 & $\begin{array}{l}\text { Psychoticism } \\
\text { (BSI) }\end{array}$ & $-.29^{\star \star}$ & $.29^{* *}$ & $.35^{\star *}$ & -.01 & -.03 & $-.31^{* *}$ & $-.22^{\star *}$ & .03 & $.42^{* *}$ & $.11^{* *}$ & $.40^{* *}$ \\
\hline 28 & $\begin{array}{l}\text { Phobic Anxiety } \\
\text { (BSI) }\end{array}$ & $-.17^{\star *}$ & $.14^{* *}$ & $.23^{\star *}$ & -.03 & .03 & $-.30^{\star *}$ & $-.15^{\star *}$ & .05 & $.44^{* *}$ & $.14^{* *}$ & $.38^{\star *}$ \\
\hline 29 & $\begin{array}{l}\text { Global Severity } \\
\text { Index (BSI) }\end{array}$ & $-.25^{\star \star}$ & $.28^{\star \star}$ & $.35^{\star *}$ & -.01 & -.02 & $-.36^{\star *}$ & $-.25^{\star *}$ & $.09^{*}$ & $.45^{\star *}$ & $.14^{* *}$ & $.46^{\star *}$ \\
\hline 30 & $\begin{array}{l}\text { AESOP } \\
\text { Mirroring }\end{array}$ & $-.12^{* *}$ & $.25^{\star *}$ & $.40^{\star *}$ & $-.09^{*}$ & $.08^{*}$ & $-.40^{* *}$ & $-.24^{* *}$ & $.36^{* *}$ & $.45^{\star *}$ & $.34^{* *}$ & $.74^{* *}$ \\
\hline 31 & $\begin{array}{l}\text { AESOP } \\
\text { Idealization }\end{array}$ & .02 & $.10^{*}$ & $.10^{* *}$ & .00 & $.11^{\star *}$ & -.01 & .00 & $.44^{* *}$ & .04 & $.54^{* *}$ & $.29^{\star *}$ \\
\hline 32 & $\begin{array}{l}\text { AESOP } \\
\text { Twinship }\end{array}$ & .04 & $.12^{* *}$ & $.15^{\star *}$ & -.05 & .02 & $-.12^{\star \star}$ & -.06 & $.72^{\star \star}$ & .02 & $.44^{* *}$ & $.42^{* *}$ \\
\hline
\end{tabular}




\begin{tabular}{|c|c|c|c|c|c|c|c|c|c|c|c|c|c|}
\hline & & 12 & 13 & 14 & 15 & 16 & 17 & 18 & 19 & 20 & 21 & 22 & 23 \\
\hline 1 & $\begin{array}{l}\text { Secure } \\
\text { Attachment } \\
(R Q)\end{array}$ & .04 & $-.19^{* *}$ & $-.23^{* *}$ & $-.11^{\star *}$ & $-.11^{* *}$ & $.18^{\star \star}$ & -.03 & $.43^{\star \star}$ & $-.17^{\star \star}$ & $-.18^{\star \star}$ & $-.22^{\star \star}$ & $-.24^{\star \star}$ \\
\hline 2 & $\begin{array}{l}\text { Fearful } \\
\text { Attachment } \\
\text { (RQ) }\end{array}$ & .00 & $.27^{\star *}$ & $.25^{\star *}$ & $.25^{\star \star}$ & $.22^{* *}$ & $-.18^{\star *}$ & .04 & $-.34^{\star \star}$ & $.16^{\star \star}$ & $.29^{* *}$ & $.26^{\star *}$ & $.28^{* *}$ \\
\hline 3 & $\begin{array}{l}\text { Preoccupied } \\
\text { Attachment } \\
\text { (RQ) }\end{array}$ & $-.08^{*}$ & $.34^{\star \star}$ & $.33^{* *}$ & $.28^{\star *}$ & $.25^{\star \star}$ & $-.31^{* *}$ & $-.08^{*}$ & $-.33^{\star *}$ & $.23^{* \star}$ & $.30^{\star *}$ & $.38^{\star \star}$ & $.34^{* *}$ \\
\hline 4 & $\begin{array}{l}\text { Dismissive } \\
\text { Attachment } \\
(\mathrm{RQ})\end{array}$ & $.19^{\star *}$ & .00 & $-.14^{\star *}$ & -.06 & -.03 & .05 & -.05 & -.02 & .01 & -.01 & $-.09^{*}$ & -.04 \\
\hline 5 & $\begin{array}{l}\text { Narcissism } \\
\text { (NPI) }\end{array}$ & .02 & .06 & $-.26^{\star *}$ & $-.17^{\star \star}$ & $-.10^{* \star}$ & $-.10^{* \star}$ & $-.58^{\star *}$ & $.17^{\star \star}$ & .03 & $-.10^{\star \star}$ & $-.12^{* \star}$ & $-.09^{*}$ \\
\hline 6 & $\begin{array}{l}\text { Self-Deception } \\
\text { (PDS) }\end{array}$ & $.16^{\star *}$ & $-.31^{* *}$ & $-.49^{* *}$ & $-.32^{\star *}$ & $-.34^{\star \star}$ & $.40^{\star *}$ & -.03 & $.34^{\star \star}$ & $-.30^{* *}$ & $-.34^{\star \star}$ & $-.35^{\star *}$ & $-.31^{* \star}$ \\
\hline 7 & $\begin{array}{l}\text { Impression } \\
\text { Management } \\
\text { (PDS) }\end{array}$ & .06 & $-.24^{* *}$ & $-.23^{* *}$ & $-.25^{\star \star}$ & $-.20^{* *}$ & $.30^{\star *}$ & $.14^{\star \star}$ & $.19^{* *}$ & $-.20^{* *}$ & $-.18^{* *}$ & $-.19^{\star *}$ & $-.23^{* *}$ \\
\hline 8 & $\begin{array}{l}\text { Approach- } \\
\text { Twinship } \\
\text { (SONI) }\end{array}$ & -.02 & $.32^{\star \star}$ & .02 & $.13^{\star \star}$ & .04 & -.04 & $-.19^{* *}$ & $.08^{*}$ & .00 & $.14^{\star *}$ & $.15^{\star *}$ & .07 \\
\hline 10 & $\begin{array}{l}\text { Avoid- } \\
\text { Idealization and } \\
\text { Twinship } \\
\text { (SONI) } \\
\text { Approach } \\
\text { Idealization } \\
\text { (SONI) }\end{array}$ & $.14^{* *}$ & $.35^{\star *}$ & $.39^{* *}$ & $.32^{* *}$ & $.31^{* *}$ & $-.33^{\star *}$ & $-.31^{* *}$ & $-.41^{\star *}$ & $.39^{* *}$ & $.30^{\star \star}$ & $.12^{* *}$ & $.40^{* *}$ \\
\hline
\end{tabular}




\begin{tabular}{|c|c|c|c|c|c|c|c|c|c|c|c|c|c|}
\hline & & 12 & 13 & 14 & 15 & 16 & 17 & 18 & 19 & 20 & 21 & 22 & 23 \\
\hline 11 & $\begin{array}{l}\text { Approach } \\
\text { Mirroring } \\
\text { (SONI) }\end{array}$ & $-.24^{* *}$ & $.58^{* *}$ & $.44^{\star *}$ & $.39^{\star *}$ & $.28^{\star \star}$ & $-.40^{* *}$ & $-.24^{* *}$ & $-.32^{* *}$ & $.32^{\star *}$ & $.40^{\star *}$ & $.47^{\star \star}$ & $.44^{* \star}$ \\
\hline 12 & $\begin{array}{l}\text { Avoid Mirroring } \\
\text { (SONI) }\end{array}$ & --- & -.04 & $-.18^{\star *}$ & -.06 & .00 & $.18^{\star \star}$ & $.12^{* \star}$ & .03 & .00 & $-.08^{*}$ & $-.13^{* *}$ & $-.09^{*}$ \\
\hline 13 & $\begin{array}{l}\text { Covert } \\
\text { Narcissism } \\
\text { (HSNS) }\end{array}$ & -.04 & --- & $.44^{\star *}$ & $.46^{* *}$ & $.37^{* \star}$ & $.43^{\star *}$ & $-.19^{* *}$ & $-.46^{\star *}$ & $.37^{\star \star}$ & $.47^{\star \star}$ & $.55^{\star \star}$ & $.49^{* *}$ \\
\hline 14 & $\begin{array}{l}\text { Self-Esteem } \\
\text { (RSES) }\end{array}$ & $-.18^{* *}$ & $.44^{* *}$ & --- & $.56^{\star *}$ & $.47^{* *}$ & $-.63^{* *}$ & $.08^{*}$ & $-.65^{\star *}$ & $.44^{* *}$ & $.54^{* *}$ & $.63^{* *}$ & $.68^{* *}$ \\
\hline 15 & Shame (PFQ2) & -.06 & $.46^{* *}$ & $.56^{* *}$ & --- & $.68^{* *}$ & $.42^{* *}$ & $.08^{*}$ & $-.44^{\star *}$ & $.43^{* \star}$ & $.53^{* *}$ & $.58^{* *}$ & $.57^{* *}$ \\
\hline 16 & Guilt (PFQ2) & .00 & $.37^{* *}$ & $.47^{* *}$ & $.68^{* *}$ & --- & $.37^{* *}$ & .03 & $-.41^{* *}$ & $.49^{* *}$ & $.51^{* *}$ & $.48^{* *}$ & $.53^{* *}$ \\
\hline 17 & $\begin{array}{l}\text { Idealization } \\
\text { (SGIS ) }\end{array}$ & $.18^{* *}$ & $.43^{* *}$ & $-.63^{* *}$ & $.42^{* *}$ & $.37^{* *}$ & --- & $-.14^{\star \star}$ & $-.54^{\star \star}$ & $.38^{* *}$ & $.53^{* *}$ & $.49^{* *}$ & $.57^{* *}$ \\
\hline 18 & $\begin{array}{l}\text { Grandiosity } \\
\text { (SGIS ) }\end{array}$ & $.12^{* *}$ & $-.19^{* *}$ & $.08^{*}$ & $.08^{*}$ & .03 & $-.14^{* \star}$ & --- & -.06 & -.04 & -.03 & .02 & -.02 \\
\hline 19 & $\begin{array}{l}\text { Social } \\
\text { Conenctedness } \\
\text { (SCS) }\end{array}$ & .03 & $-.46^{* *}$ & $-.65^{* *}$ & $-.44^{\star *}$ & $-.41^{\star *}$ & $-.54^{* *}$ & -.06 & --- & $-.45^{\star *}$ & $-.51^{* *}$ & $-.56^{* *}$ & $-.60^{* *}$ \\
\hline 20 & $\begin{array}{l}\text { Somatization } \\
\text { (BSI) }\end{array}$ & .00 & $.37^{* *}$ & $.44^{* *}$ & $.43^{* *}$ & $.49^{* *}$ & $.38^{* *}$ & -.04 & $-.45^{* *}$ & --- & $.64^{* *}$ & $.60^{* *}$ & $.66^{* *}$ \\
\hline 21 & $\begin{array}{l}\text { Obsessive- } \\
\text { Compulsize } \\
\text { (BSI) }\end{array}$ & $-.08^{*}$ & $.47^{* *}$ & $.54^{* *}$ & $.53^{* \star}$ & $.51^{\star \star}$ & $.53^{* \star}$ & -.03 & $-.51^{\star \star}$ & $.64^{\star *}$ & --- & $.65^{\star *}$ & $.71^{* *}$ \\
\hline
\end{tabular}




\begin{tabular}{|c|c|c|c|c|c|c|c|c|c|c|c|c|c|}
\hline & & 12 & 13 & 14 & 15 & 16 & 17 & 18 & 19 & 20 & 21 & 22 & 23 \\
\hline 22 & $\begin{array}{l}\text { Interpersonal } \\
\text { Sensitivty (BSI) }\end{array}$ & $-.13^{\star *}$ & $.55^{\star \star}$ & $.63^{\star *}$ & $.58^{\star \star}$ & $.48^{* *}$ & $.49^{\star *}$ & .02 & $-.56^{* *}$ & $.60^{\star *}$ & $.65^{\star *}$ & --- & $.77^{* *}$ \\
\hline 23 & $\begin{array}{l}\text { Depression } \\
\text { (BSI) }\end{array}$ & $-.09^{*}$ & $.49^{* *}$ & $.68^{\star *}$ & $.57^{\star \star}$ & $.53^{\star \star}$ & $.57^{\star *}$ & -.02 & $-.60^{* *}$ & $.66^{\star \star}$ & $.71^{\star *}$ & $.77^{* \star}$ & --- \\
\hline 24 & Anxiety (BSI) & $-.08^{*}$ & $.47^{* *}$ & $.53^{* *}$ & $.56^{\star \star}$ & $.55^{\star *}$ & $.47^{* *}$ & -.05 & $-.50^{\star *}$ & $.78^{\star \star}$ & $.73^{\star *}$ & $.69^{* *}$ & $.73^{\star *}$ \\
\hline 25 & Hostility (BSI) & -0.02 & $.45^{\star *}$ & $.47^{* *}$ & $.45^{\star *}$ & $.47^{* *}$ & $.40^{* *}$ & $-.11^{* *}$ & $-.47^{\star *}$ & $.68^{\star \star}$ & $.62^{* *}$ & $.62^{\star *}$ & $.70^{\star *}$ \\
\hline 26 & $\begin{array}{l}\text { Paranoid } \\
\text { Ideation (BSI) }\end{array}$ & -.06 & $.56^{\star \star}$ & $.48^{* *}$ & $.48^{\star *}$ & $.50^{* *}$ & $.40^{* *}$ & $-.16^{\star *}$ & $-.52^{\star *}$ & $.61^{* *}$ & $.66^{\star *}$ & $.72^{\star *}$ & $.70^{\star * *}$ \\
\hline 27 & $\begin{array}{l}\text { Psychoticism } \\
\text { (BSI) }\end{array}$ & -.07 & $.50^{\star *}$ & $.61^{* *}$ & $.56^{\star \star}$ & $.55^{\star *}$ & $.49^{\star *}$ & -.03 & $-.60^{\star *}$ & $.72^{\star \star}$ & $.71^{* *}$ & $.73^{\star \star}$ & $.84^{\star *}$ \\
\hline 28 & $\begin{array}{l}\text { Phobic Anxiety } \\
\text { (BSI) }\end{array}$ & -.04 & $.40^{\star *}$ & $.44^{\star *}$ & $.44^{\star *}$ & $.44^{\star *}$ & $.36^{\star \star}$ & -.03 & $-.45^{\star *}$ & $.76^{\star \star}$ & $.59^{\star *}$ & $.66^{\star *}$ & $.61^{* *}$ \\
\hline 29 & $\begin{array}{l}\text { Global Severity } \\
\text { Index (BSI) }\end{array}$ & -.07 & $.55^{\star *}$ & $.63^{* *}$ & $.60^{* *}$ & $.59^{* *}$ & $.54^{\star *}$ & -.06 & $-.61^{\star *}$ & $.85^{\star \star}$ & $.83^{\star *}$ & $.83^{\star \star}$ & $.88^{* *}$ \\
\hline 30 & $\begin{array}{l}\text { AESOP } \\
\text { Mirroring }\end{array}$ & $-.22^{\star \star}$ & $.62^{\star *}$ & $-.55^{\star \star}$ & $.45^{\star *}$ & $.35^{\star *}$ & $.46^{\star *}$ & $-.22^{* *}$ & $-.45^{\star \star}$ & $.37^{\star *}$ & $.46^{\star *}$ & $.57^{\star *}$ & $.55^{\star \star}$ \\
\hline 31 & $\begin{array}{l}\text { AESOP } \\
\text { Idealizing }\end{array}$ & .02 & $.21^{\star *}$ & -.00 & $.12^{* *}$ & .05 & .05 & $-.23^{\star *}$ & -.10 & .00 & $.11^{* *}$ & $.09^{*}$ & .06 \\
\hline 32 & $\begin{array}{l}\text { AESOP } \\
\text { Twinship }\end{array}$ & -.06 & $.33^{* *}$ & $-.10^{* *}$ & $.17^{\star *}$ & $.09^{*}$ & $.10^{* *}$ & $-.20^{* *}$ & -.08 & .03 & $.19^{\star *}$ & $.20^{* *}$ & $.16^{\star *}$ \\
\hline
\end{tabular}




\begin{tabular}{|c|c|c|c|c|c|c|c|c|c|c|}
\hline & & 24 & 25 & 26 & 27 & 28 & 29 & 30 & 31 & 32 \\
\hline 1 & $\begin{array}{l}\text { Secure } \\
\text { Attachment } \\
(\mathrm{RQ})\end{array}$ & $-.18^{* *}$ & $-.24^{\star *}$ & $-.30^{* *}$ & $-.29^{* *}$ & $-.17^{\star *}$ & $-.25^{\star \star}$ & $-.12^{\star \star}$ & .02 & .04 \\
\hline 2 & $\begin{array}{l}\text { Fearful } \\
\text { Attachment } \\
(\mathrm{RQ})\end{array}$ & $.22^{* *}$ & $.23^{* *}$ & $.32^{\star *}$ & $.29^{* *}$ & $.14^{\star *}$ & $.28^{* *}$ & $.25^{\star *}$ & $.10^{*}$ & $.12^{\star *}$ \\
\hline 3 & $\begin{array}{l}\text { Preoccupied } \\
\text { Attachment } \\
\text { (RQ) }\end{array}$ & $.29^{\star *}$ & $.26^{* *}$ & $.32^{* *}$ & $.35^{\star *}$ & $.23^{* *}$ & $.35^{\star *}$ & $.40^{* *}$ & $.10^{* *}$ & $.15^{\star *}$ \\
\hline 4 & $\begin{array}{l}\text { Dismissive } \\
\text { Attachment } \\
(\mathrm{RQ})\end{array}$ & -.02 & .04 & .01 & -.01 & -.03 & -.01 & $-.09^{*}$ & .00 & -.05 \\
\hline 5 & $\begin{array}{l}\text { Narcissism } \\
\text { (NPI) }\end{array}$ & -.02 & .05 & $.07^{*}$ & -.03 & .03 & -.02 & $.08^{*}$ & $.11^{* *}$ & .02 \\
\hline 6 & $\begin{array}{l}\text { Self- } \\
\text { Deception } \\
\text { (PDS) }\end{array}$ & $-.34^{\star *}$ & $-.28^{* *}$ & $-.25^{* *}$ & $-.31^{* *}$ & $-.30^{* *}$ & $-.36^{\star *}$ & $-.40^{\star *}$ & -.01 & $-.12^{\star \star}$ \\
\hline 7 & $\begin{array}{l}\text { Impression } \\
\text { Management } \\
\text { (PDS) }\end{array}$ & $-.22^{* *}$ & $-.26^{\star *}$ & $-.20^{* *}$ & $-.22^{* \star}$ & $-.15^{\star *}$ & $-.25^{\star *}$ & $-.24^{\star *}$ & .00 & -.06 \\
\hline 8 & $\begin{array}{l}\text { Approach- } \\
\text { Twinship } \\
\text { (SONI) }\end{array}$ & $.07^{*}$ & $.08^{*}$ & $.14^{* *}$ & .03 & .05 & $.09^{*}$ & $.36^{\star *}$ & $.44^{\star *}$ & $.72^{\star \star}$ \\
\hline 10 & $\begin{array}{l}\text { Avoid- } \\
\text { Idealization } \\
\text { and Twinship } \\
\text { (SONI) } \\
\text { Approach } \\
\text { Idealization } \\
\text { (SONI) }\end{array}$ & $.42^{* *}$ & $.40^{* *}$ & $.16^{\star \star}$ & $.11^{* *}$ & $.14^{\star \star}$ & $.14^{\star \star}$ & $.34^{\star *}$ & $.54^{\star \star}$ & $.44^{\star *}$ \\
\hline
\end{tabular}




\begin{tabular}{|c|c|c|c|c|c|c|c|c|c|c|}
\hline & & 24 & 25 & 26 & 27 & 28 & 29 & 30 & 31 & 32 \\
\hline 11 & $\begin{array}{l}\text { Approach } \\
\text { Mirroring } \\
\text { (SONI) }\end{array}$ & $.40^{* *}$ & $.34^{* *}$ & $.44^{* *}$ & $.40^{* *}$ & $.38^{* *}$ & $.46^{* *}$ & $.74^{\star *}$ & $.29^{\star *}$ & $.42^{* *}$ \\
\hline 12 & $\begin{array}{l}\text { Avoid Mirroring } \\
\text { (SONI) }\end{array}$ & $-.08^{*}$ & -.02 & -.06 & -.06 & -.04 & -.07 & $-.22^{\star \star}$ & .02 & -.06 \\
\hline 13 & $\begin{array}{l}\text { Covert } \\
\text { Narcissism } \\
\text { (HSNS) }\end{array}$ & $.47^{* *}$ & $.45^{* *}$ & $.56^{* *}$ & $.50^{* *}$ & $.40^{* *}$ & $.55^{* *}$ & $.62^{* *}$ & $.21^{* *}$ & $.33^{\star *}$ \\
\hline 14 & $\begin{array}{l}\text { Self-Esteem } \\
\text { (RSES) }\end{array}$ & $.53^{* *}$ & $.47^{* *}$ & $.48^{* *}$ & $.61^{* *}$ & $.44^{* *}$ & $.63^{* *}$ & $-.55^{\star \star}$ & .00 & $-.10^{* *}$ \\
\hline 15 & Shame (PFQ2) & $.56^{* *}$ & $.45^{* *}$ & $.48^{* *}$ & $.56^{* *}$ & $.44^{* *}$ & $.60^{* *}$ & $.45^{\star *}$ & $.12^{* *}$ & $.17^{\star *}$ \\
\hline 16 & Guilt (PFQ2) & $.55^{* *}$ & $.47^{* *}$ & $.50^{* *}$ & $.55^{* *}$ & $.44^{* *}$ & $.59^{* *}$ & $.35^{\star \star}$ & .05 & $.09^{*}$ \\
\hline 17 & $\begin{array}{l}\text { Idealization } \\
\text { (SGIS ) }\end{array}$ & $.47^{* *}$ & $.40^{* *}$ & $.40^{* *}$ & $.49^{* *}$ & $.36^{* *}$ & $.54^{* *}$ & $.46^{\star \star}$ & .05 & $.10^{* *}$ \\
\hline 18 & $\begin{array}{l}\text { Grandiosity } \\
\text { (SGIS ) }\end{array}$ & -.05 & $-.11^{* *}$ & $-.16^{* *}$ & -.03 & -.03 & -.06 & $-.22^{\star \star}$ & $-.23^{* *}$ & $-.2^{* *}$ \\
\hline 19 & $\begin{array}{l}\text { Social } \\
\text { Conenctedness } \\
\text { (SCS) }\end{array}$ & $-.50^{* *}$ & $-.47^{* *}$ & $-.52^{* *}$ & $-.60^{* *}$ & $-.45^{\star *}$ & $-.61^{* *}$ & $-.45^{\star *}$ & -.10 & -.08 \\
\hline 20 & $\begin{array}{l}\text { Somatization } \\
\text { (BSI) }\end{array}$ & $.78^{* *}$ & $.68^{* *}$ & $.61^{* *}$ & $.72^{* *}$ & $.76^{* *}$ & $.85^{* *}$ & $.37^{* *}$ & .00 & .03 \\
\hline 21 & $\begin{array}{l}\text { Obsessive- } \\
\text { Compulsize } \\
\text { (BSI) }\end{array}$ & $.73^{* *}$ & $.62^{* *}$ & $.66^{* *}$ & $.71^{* *}$ & $.59^{* *}$ & $.83^{* *}$ & $.46^{\star *}$ & $.11^{* *}$ & $.19^{* *}$ \\
\hline
\end{tabular}




\begin{tabular}{|c|c|c|c|c|c|c|c|c|c|c|}
\hline & & 24 & 25 & 26 & 27 & 28 & 29 & 30 & 31 & 32 \\
\hline 22 & $\begin{array}{l}\text { Interpersonal } \\
\text { Sensitivty (BSI) }\end{array}$ & $.69^{* *}$ & $.62^{* *}$ & $.72^{\star *}$ & $.73^{\star *}$ & $.66^{* *}$ & $.83^{\star *}$ & $.57^{\star *}$ & $.09^{*}$ & $.20^{* *}$ \\
\hline 23 & $\begin{array}{l}\text { Depression } \\
\text { (BSI) }\end{array}$ & $.73^{\star *}$ & $.70^{\star *}$ & $.70^{\star *}$ & $.84^{\star *}$ & $.61^{* *}$ & $.88^{\star *}$ & $.55^{\star *}$ & .06 & $.16^{* *}$ \\
\hline 24 & Anxiety (BSI) & --- & $.70^{\star *}$ & $.68^{* *}$ & $.75^{\star \star}$ & $.76^{* *}$ & $.89^{* *}$ & $.48^{* *}$ & $.09^{*}$ & $.13^{* *}$ \\
\hline 25 & Hostility (BSI) & $.70^{* *}$ & --- & $.68^{\star *}$ & $.71^{* *}$ & $.64^{\star *}$ & $.82^{* *}$ & $.44^{\star *}$ & .04 & $.11^{* *}$ \\
\hline 26 & $\begin{array}{l}\text { Paranoid } \\
\text { Ideation (BSI) }\end{array}$ & $.68^{\star *}$ & $.68^{\star *}$ & --- & $.73^{\star *}$ & $.63^{\star *}$ & $.82^{\star *}$ & $.55^{\star *}$ & $.09^{*}$ & $.20^{* *}$ \\
\hline 27 & $\begin{array}{l}\text { Psychoticism } \\
\text { (BSI) }\end{array}$ & $.75^{\star *}$ & $.71^{\star *}$ & $.73^{\star *}$ & --- & $.71^{\star *}$ & $.90^{\star *}$ & $.50^{\star *}$ & .06 & $.10^{\star *}$ \\
\hline 28 & $\begin{array}{l}\text { Phobic Anxiety } \\
\text { (BSI) }\end{array}$ & $.76^{\star *}$ & $.64^{\star *}$ & $.63^{* *}$ & $.71^{* *}$ & --- & $.82^{* *}$ & $.39^{\star *}$ & .04 & $.08^{*}$ \\
\hline 29 & $\begin{array}{l}\text { Global Severity } \\
\text { Index (BSI) }\end{array}$ & $.89^{* *}$ & $.82^{* *}$ & $.82^{* *}$ & $.90^{* *}$ & $.82^{* *}$ & --- & $.55^{\star *}$ & .07 & $.15^{\star *}$ \\
\hline 30 & $\begin{array}{l}\text { AESOP } \\
\text { Mirroring }\end{array}$ & $.48^{* *}$ & $.44^{* *}$ & $.55^{\star *}$ & $.50^{\star *}$ & $.39^{* *}$ & $.55^{\star *}$ & --- & $.31^{* *}$ & $.44^{\star *}$ \\
\hline 31 & $\begin{array}{l}\text { AESOP } \\
\text { Idealizing }\end{array}$ & $.09^{*}$ & .04 & $.09^{*}$ & .06 & .04 & .07 & $.31^{* *}$ & --- & $.44^{* *}$ \\
\hline
\end{tabular}




\section{APPENDIX A}

\section{Instructions}

Instructions: These are questions concerning your thoughts and feelings about yourself and your relationships with others.

Please read each statement carefully and decide how much the statement is generally true of you on a scale from: $1=$ "Not at all true of me"; to 7 = "Very true of me".

Be sure to answer every item and try to be as honest and accurate as possible in your responses.

\section{I try to surround myself with successful people.}

2

23

4

5

6

7

Not at all

Very true

true of me

of me

2. Sometimes I feel like I have a special connection with famous people.

$\begin{array}{llllllll}1 & 2 & 3 & 4 & 5 & 6 & 7\end{array}$

Not at all

Very true

true of me

of me

3. There is at least one person in my life that I look to as a model of how I would like to be.

1 2 3 4 5 6 7

Not at all

Very true

true of me of me

4. When I need help, I feel better when there is someone in authority who can manage things for me.

$\begin{array}{llllllll}1 & 2 & 3 & 4 & 5 & 6 & 7\end{array}$

Not at all

Very true

true of me

of me 
5. I am cynical about authority figures and powerful people.*

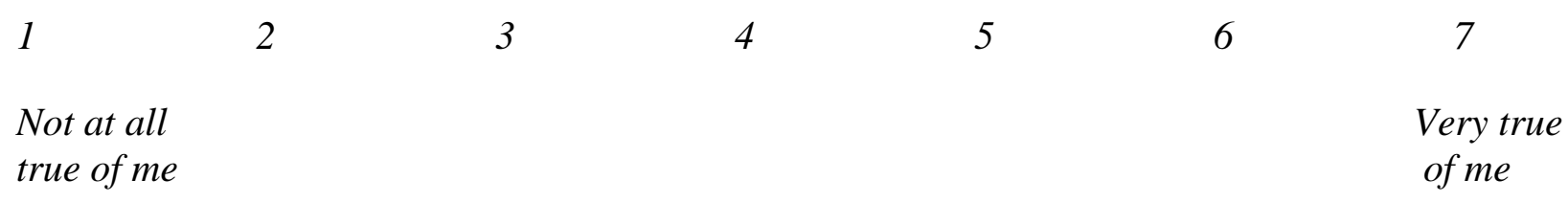

6. Whenever I can, I try to spend time with people that I look up to.

$\begin{array}{llllllll}1 & 2 & 3 & 4 & 5 & 6 & 7\end{array}$

Not at all

true of me

Very true

of me

7. Without leaders who can give us courage, the world is a scary place.
1
2
3
4
5
6
7
Not at all
true of me
Very true of me

8. I feel better when I have someone to serve as my mentor.

$\begin{array}{llllllll}1 & 2 & 3 & 4 & 5 & 6 & 7\end{array}$

Not at all

true of me

Very true

of me

9. I feel empty inside when the people I look up to are not available.
1
23
4
5
6
7
Not at all
true of me
Very true of me

10. I feel safe when I am around people who are calm and confident.
1
23
4
5
6
7
Not at all
true of me
Very true of me 
11. I do not like it when people try to give me advice.

1 2 3

4 5

6 7

Not at all

true of me

Very true of me

12. It's important to have people that you admire in your life.

1

Not at all

true of me
2

3 5

6 7

Very true of me

13. It hurts when the people I look up to don't live up to my expectations.

1 2 3

4 5 6

Not at all

true of me

Very true of me

14. I try hard to emulate people who I think highly of.

1 2 3

4 5 6 7

Not at all true of me

15. I believe that the way to get ahead in life is to follow in the footsteps of great people.

$\begin{array}{lllllll}1 & 2 & 3 & 4 & 5 & 6 & 7\end{array}$

Not at all

Very true

true of me

of me 
16. When I'm away from the people that I think highly of, I feel empty inside. 2 3

4 5

6 7

Not at all Very true true of me of me

17. I feel better when I'm in the presence of wise people.

$\begin{array}{llllllll}1 & 2 & 3 & 4 & 5 & 6 & 7\end{array}$

Not at all

Very true

true of me

of me

18. I feel fragile when I don't have strong people in my life to lean upon.

1

2

3

4

5

6

7

Not at all

Very true

true of me

of me

19. I sometimes dream or fantasize about meeting famous or influential people.

1

2

3

4

5

6

7

Not at all

Very true

true of me

of me

20. I want to be close to people who have some special talent, skill, or wisdom about them.

1

2

3

4

5

6

7

Not at all

Very true

true of me

of me

21. I tend to put people on a pedestal.

1

2

3

4

5

6

7

Not at all

true of me
Very true of me 
22. When I'm challenged or stressed, I think of how a hero would act.

1 2 3

4 5

6 7

Not at all

true of me

Very true of me

23. I tend to have faith in authority figures.

$\begin{array}{llllllll}1 & 2 & 3 & 4 & 5 & 6 & 7\end{array}$

Not at all

Very true

true of me

of me

24. I like to figure things out for myself without "expert" guidance.

$\begin{array}{llllllll}1 & 2 & 3 & 4 & 5 & 6 & 7\end{array}$

Not at all

Very true

true of me

of me

25. It's devastating when I'm disappointed by the people in my life that I respect.

$\begin{array}{llllllll}1 & 2 & 3 & 4 & 5 & 6 & 7\end{array}$

Not at all

Very true

true of me

of me

26. It's important to me that my achievements or contributions are recognized.

2

3

4

6

7

Not at all

Very true

true of me

of me 
27.I feel that people do not appreciate the struggles I've had to face. 2

3

4 5

6 7

Not at all

Very true

true of me

of me

28. When people don't recognize my accomplishments, I feel insecure.

$\begin{array}{llllllll}1 & 2 & 3 & 4 & 5 & 6 & 7\end{array}$

Not at all

Very true

true of me

of me

29. I want people to hear and care about the things that are going on in my life.

1 2 3

4 5

6 7

Not at all

true of me

Very true of me

30. I feel better when others appreciate me.

1 2 3

4 5

6 7

Not at all

true of me

Very true of me

31. I want to be well thought of by those around me.

1

2

3

4

5

6

7

Not at all

Very true

true of me

of me 
32. I feel unappreciated.

1

Not at all

true of me
23

4

5

6

7

Very true

of me

33. I don't care if other people are interested in me.
2

3

4

5

6

7

Not at all

true of me
Very true

of me

34. When I am doing well at something, I need people to realize that I'm doing a good job.

1

2

3

4

5

6

7

Not at all

true of me
Very true

of me

35. When I'm around someone who cares about what I'm doing, I feel better about myself.

1

2

3

4

5

6

7

Not at all

true of me
Very true of me

36. I'm devastated when people criticize me or my contributions.

$\begin{array}{llllllll}1 & 2 & 3 & 4 & 5 & 6 & 7\end{array}$

Not at all

Very true

true of me

of me

37. I feel that others do not really listen to me.
1
2
3
4
5
6
7
Not at all
Very true
true of me
of me 
38. I worry that people don't care about me or my life.

1

Not at all

true of me

23

4

5

6 7

Very true of me

39. I wish people knew my value and understood my contributions better.

1 2 3

4

5

6

7

Not at all

true of me

Very true

of me

40. Life would be a lot better if people appreciated me.

$$
1
$$

$$
2
$$

3

4

5

6

7

Not at all

true of me

41. It's important to me that people acknowledge the work that I do.

1 2 3

4

5

6

7

Not at all

true of me

42. I feel empty inside if people don't care about what I'm doing.

1

Not at all

true of me
23

4

5

6

7

Very true

of me

Very true of me

43. I crave the admiration of other people.

1

2

3

4

5

6

7

Not at all

true of me

Very true of me 
44. If people don't care about what I'm doing, I find it hard to keep going.

$\begin{array}{llllllll}1 & 2 & 3 & 4 & 5 & 6 & 7\end{array}$

Not at all

true of me

Very true

of me

45. I dream or fantasize about being recognized for the work that I do.

$\begin{array}{llllllll}1 & 2 & 3 & 4 & 5 & 6 & 7\end{array}$

Not at all

Very true

true of me

of me

46. I feel as though people in my life haven't celebrated my accomplishments.

$\begin{array}{lllllll}1 & 2 & 3 & 4 & 5 & 6 & 7\end{array}$

Not at all

Very true

true of me

of me

47. I prefer to go unnoticed.

$\begin{array}{llllllll}1 & 2 & 3 & 4 & 5 & 6 & 7\end{array}$

Not at all

Very true

true of me

of me

48. There is at least one person in my life who appreciates me and what I do.

1

2

3

4

5

6

7

Not at all

Very true

true of me

of me 
49. I wish people knew that I need their recognition -- not just their help.

1 2

3

4 5

6

7

Not at all

Very true

true of me

of me

50. It helps me to be around people going through the same things that I am going through.

1

Not at all

true of me
2

3

4

5

6

7

Very true

of me

51. I like talking with people who have opinions similar to mine.

$\begin{array}{llllllll}1 & 2 & 3 & 4 & 5 & 6 & 7\end{array}$

Not at all

Very true

true of me

of me

52. I like knowing that my feelings are shared by others.

$\begin{array}{llllllll}1 & 2 & 3 & 4 & 5 & 6 & 7\end{array}$

Not at all

Very true

true of me

of me

53. It's easier for me to handle stress when I know that other people are going through the same thing.
1
2
3
4
5
6
7
Not at all
Very true
true of me
of me 
54. I want my friends to share my beliefs.

1

Not at all

true of me
$2 \quad 3 \quad 4$

3

5

6

Very true

of me

55. I always wanted a friend who is just like me.

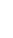

2

3

4

5

6

7

Not at all

true of me

56. I feel uncomfortable around people who seem too similar to me.

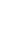
2

3

4

5

6

7

Very true

of me

of me

Not at all

true of me

57. When I'm stressed, I seek advice from people who are in the same situation. 2

3

4

5

6

Not at all

Very true

true of me

of me

58. I dream or fantasize about having friends who are like me.

2

3

4

5

6

7

Not at all

true of me

Very true

of me 
59. I feel empty inside when I don't have peers around who are like me.

1

Not at all

true of me

2

3

4

5

6

Very true

of me

60. I like being part of a team or a group.

1 2

3

4

5

6

7

Not at all

true of me

61. I get angry when my friends do not agree with my values and beliefs.

$\begin{array}{llllllll}1 & 2 & 3 & 4 & 5 & 6 & 7\end{array}$

Not at all

true of me

Very true

of me

Very true

of me

7

true

62. I like to standout, be different, or choose a different path from my peers.

$\begin{array}{llllllll}1 & 2 & 3 & 4 & 5 & 6 & 7\end{array}$

Not at all

Very true

true of me

of me

63. Being around people who are similar to me keeps me feeling grounded.
1
2
3
4
5
6
7
Not at all
true of me
Very true
of me 
64. I am happiest when I feel like I am part of a community of likeminded people.

1

Not at all

true of me

2

3

4

5

6

7

Very true

of me

65. It is hard for me to connect with people who are different from me.

2

3

4

5

6

7

Not at all

Very true

true of me

of me

66. Being around people who think like I do makes it easier to overcome difficulties or solve problems.

$\begin{array}{llllllll}1 & 2 & 3 & 4 & 5 & 6 & 7\end{array}$

Not at all

Very true

true of me

of me

67. I seek out people who are different from me to have as friends.

$\begin{array}{llllllll}1 & 2 & 3 & 4 & 5 & 6 & 7\end{array}$

Not at all

Very true

true of me

of me

68. I prefer to talk with people who challenge my beliefs and values.

$\begin{array}{llllllll}1 & 2 & 3 & 4 & 5 & 6 & 7\end{array}$

Not at all

Very true

true of me

of me 
69. I have at least one friend who I feel is like me.

1

2

3

4

5

6

Not at all

Very true

true of me

of me

70. I feel fragile when I do not have someone like me to talk to.

1

2

3

4

5

6

7

Not at all

true of me

Very true

of me 


\section{REFERENCES}

Ainsworth, M. D. S. (1967). Infancy in Uganda: Infant care and the growth of love. Baltimore, MD: Johns Hopkins University Press.

Ainsworth, M. D. S., \& Bell, S. M. (1970). Attachment, exploration, and separation: Illustrated by the behavior of one-year-olds in a strange situation. Child Development, 41, 49-67.

Ainsworth, M. D. S., Bell, S., \& Stayton, D. (1971). Individual differences in strange situation behavior of one-year-olds. In H. R. Shaffer (Ed.), The origins of human social relations. London \& New York: Academic Press.

Ainsworth, M. D. S., Blehar, M. C., Waters, E., \& Wall, S. (1978). Patterns of Attachment. A Psychological Study of the Strange Situation. Hillsdale, NJ: Lawrence Erlbaum Associates.

Ashby, H., Lee, R., \& Duke, E., (1979). A narcissistic personality disorder MMPI scale. Paper presented at the 87th annual meeting of the American Psychological Association, New York, NY.

Baker, H. \& Baker, M. 1987. Heinz Kohut's self psychology: An overview. American Journal of Psychiatry, 144, 1-9.

Banai, E., Mikulincer, M., \& Shaver, P. R. (2005). "Selfobject" needs in Kohut's self psychology: Links with attachment, self-cohesion, affect regulation, and adjustment. Psychoanalytic Psychology, 22(2), 224-260.

Barry, C. T., Frick, P. J., Adler, K. K., \& Grafemen, S. J. (2007). The predictive utility of narcissism among children and adolescents: Evidence of a distinction between adaptive and maladaptive narcissism. Journal of Child and Family Studies, 16, 508-521. 
Barth, F. (1988). The treatment of bulimia from a self psychological perspective. Clinical Social Work Journal, 16, 270-281.

Bartlett, M. S., (1954). A note on the multiplying factors for various chi-square approximations. Journal of the Royal Statistical Society, 16 (Series B), 296-298.

Bartholomew, K., \& Horowitz, L. M. (1991). Attachment styles among young adults: A test of a four-category model. Journal of Personality \& Social Psychology, 61, 226 - 244.

Basch, M. (1992). Practicing psychotherapy: The science behind the art. New York: Basic Books.

Bastanfar, R. B.(2009). An examination of the relationship between psychoanalytic development of the self and attributions and expectations for events: An attempt at bridging positive psychology and Kohut's self-psychology (Unpublished doctoral dissertation). Michigan State University, East Lansing.

Beck, J. S. (1992). Cognitive Therapy: Basics and Beyond. New York: The Guildford Press.

Beebe, B., \& Lachman, F.M. (1988). The contribution of mother-infant mutual influence to the origins of the self-and object relationships. Psychoanalytic Psychology, 5, 305-337.

Besser, A., \& Priel, B. (2009). Emotional responses to a romantic partner's imaginary rejection: The roles of attachment anxiety, covert narcissism, and self-evaluation. Journal of Personality, 77, 287-325.

Biscardi, D., \& Schill, T. (1985). Correlations of narcissistic traits with defensive style, Machiavellianism, and empathy. Psychological Reports, 57, 354.

Blashfield, R. K., \& Intoccia, V. (2000). Growth of the literature on the topic of personality disorders. American Journal of Psychiatry, 157, 472-473. 
Bohlin, G., Hagekull, B., \& Rydell, A. M. (2000). Attachment and Social Functioning: A Longitudinal Study from Infancy to Middle Childhood. Social Development, 9(1), 24-39. Bollen, KA. (1989). Structural Equations with Latent Variables. Wiley: New York, NY.

Bowlby, J. (1958). The nature of the child's tie to its mother. International Journal of PsychoAnalysis, 39, 350-373.

Bowlby, J, (1969). Attachment and loss: Volume 1. Attachment. New York: Basic Books.

Bowlby, J. (1973). Attachment and loss: Volume 2. Separation: Anxiety and Anger. New York: Basic Books.

Bowlby, J. (1980). Attachment and loss: Volume 3. Loss: Sadness and depression. New York: Basic Books.

Bowlby, J. (1988). A Secure Base. New York: Basic Books.

Brennan, K. A., Clark, C. L., \& Shaver, P. R. (1998). Self-report measurement of adult romantic attachment: An integrative overview. In J. A. Simpson \& W. S. Rholes (Eds.), Attachment theory and close relationships (pp. 46-76). New York: Guilford.

Broucek, F. (1991). Shame and the self. New York: Guilford.

Canepa, H. (2011). Need for belonging and attachment style in relation to college students' participation in negative group initiation practices (Unpublished doctoral dissertation). Pace university, New York.

Carducci, B. J. (2009). The psychology of personality: Viewpoints, research, and applications. (2nd ed.) West Sussex, United Kingdom: Wiley.

Carlson, E. A. (1998). A prospective longitudinal study of disorganized/disoriented attachment. Child Development, 69, 1970-1979. 
Carlson, E. A., Sroufe, L. A., \& Egeland, B. (2004). The construction of experience: A longitudinal study of representation and behavior. Child Development, 75, 66-83.

Carr, E., \& Cortina, M. (2011). Heinz Kohut and John Bowlby: The Men and Their Ideas. Psychoanalytic Inquiry, 31, 42-57.

Cassidy, J. (1994). Emotion regulation: Influences of attachment relationships. In N. Fox (Ed.), The development of emotion regulation: Biological and biobehavioral considerations. Monographs of the Society for Research in Child Development 59 (2-3 Serial No. 240).

Chenot, D. K. (1998). Mutual values: Self psychology, intersubjectivity, and social work. Clinical Social Work Journal, 26(3), 297-311.

Cooper, A. (1998). Disorders of narcissism: Diagnostic, clinical, and empirical implications. Washington, DC: American Psychiatric Press.

Cortina, M., \& Liotti, G. (2010). Attachment is about safety and protection. Intresubjectivity is about social understanding and sharing. The relationships between attachment and intersubjectivity. Psychoanalytic Psychology, 27(4), 410-441.

Costa, P. T., \& McCrae, R. R. (1992). Revised NEO Personality Inventory (NEO-PI-R) and NEO Five-Factor Inventory (NEO-FFI) professional manual. Lutz, FL: PAR.

Costello, A. B., \& Osborne, J. W. (2005). Best practices in exploratory factory analysis: Four recommendations for getting the most from your analysis. Practical Assessment, Research, \& Evaluation, 10(7). Available online: http://pareonline.net/getvn.asp?v=10\&n=7.

Cyr, C., Euser, E. M., Bakermans-Kranenburg, M. J., \& van Ijzendoorn, M. H. (2010). Attachment and security and disorganization in maltreating and high-risk families: A series of meta-analyses. Development and Psychopathology, 22, 87-108. 
De Vaus, D. (2002). Analyzing social science data: 50 key problems in data analysis. Los Angeles, CA: Sage.

Dickinson, K. A., \& Pincus, A. L. (2005). Interpersonal analysis of grandiose and vulnerable narcissism. Journal of Personality Disorders, 17(3), 188-207.

Elliott, R. Rohart, A.C., Watson, J. C., \& Greenberg, L. S. (2011). Empathy. Psychotherapy, 48(1), 43-49.

Emmons, R. (1984). Factor analysis and construct validity of the narcissistic personalityinventory. Journal of Personality and Social Psychology, 48, 291-300.

Exner, J.E. (2003). The Rorschach: A comprehensive system. Vol. 1: Fourth Edition. New York: Wiley.

Farmer, R., \& Sundberg, N. D. (1986). Boredom proneness-The development and correlates of a new scale. Journal of Personality Assessment, 50, 4-17.

Fenchel, G. (1983). Interaction between narcissism and masochism in the borderline personality. Issues in Ego Psychology, 6, 62-68.

Figley, C. R. (2002), Compassion fatigue: Psychotherapists' chronic lack of self care. Journal of Clinical Psychology, 58: 1433-1441. doi: 10.1002/jclp.10090

Freud, S. (1914). On narcissism: An introduction. In A.P. Morrison (Ed.). (1986).

Freud, S. (1927/1961). The ego and the id. In Standard edition of The complete psychological works of Sigmund Freud, vol. 19, ed. J. Strachey and A. Freud, 3-66. London: Hogarth Press.

Gabbard, G. (2000). Psychodynamic psychiatry in clinical practice (3rd ed.). Washington, DC: American Psychiatric Press. 
Gabbard, G. (1983). Further contributions to the understanding of stage fright: narcissistic issues. Journal of American Psychoanalytic Association, 31, 423-441.

Garfield, D. (2001). The use of vitality affects in the coalescence of self in psychosis. In A. Goldberg, (ed.), Progress in Self Psychology, Volume 17. Hillsdale, NJ: Analytic Press, 113-128.

George, C., Kaplan, N., \& Main, M. (1984). Adult Attachment Interview protocol: (Unpublished manuscript). University of California, Berkeley.

Giugliano, J. R. (2011). Self-psychological treatment of a sex addict with sadomasochistic behaviours: A case report. British Journal of Psychotherapy, 27: 352-367.

Gleason, T. R., Jarudi, R. N., \& Cheek, J. M. (2003). Imagination, personality, and imaginary companions. Social Behavior and Personality, 31(7), 721-738

Goldman, G. F. \& Gelso, C. J. (1997). Kohut's theory of narcissism and adolescent drug abuse treatment. Psychoanalytic Psychology, 14(1), 81-94.

Gomez, L. (1997). An Introduction to Object Relations. London: Free Association.

Gruber, D. B. (2009). Overt and covert narcissism, anger, and selfobject needs (Unpublished doctoral dissertation). Richard R. Conolly College of Long Island University, Brooklyn.

Gunderson, J., G., Ronningstam, E., \& Bodkin, A. (1987). The Diagnostic Interview for Narcissistic Patients. Arch Gen Psychiatry, 47(7), 676-680.

Hahn, D. C. (1994). God as mirroring and idealizing selfobject function - its influence on selfcohesion and mood: An empirical study. (Unpublished doctoral dissertation). The California School of Professional Psychology, San Diego.

Hamilton, C. E. (2000). Continuity and discontinuity of attachment from infancy to adolescence. Child Development, 71, 690-694. 
Hartmann, H.-P. (2009), Psychoanalytic Self Psychology and Its Conceptual Development in Light of Developmental Psychology, Attachment Theory, and Neuroscience. Annals of the New York Academy of Sciences, 1159: 86-105.

Hayton, J. C., Allen, D. G., \& Scarpello, V. (2004). Factor retention decisions in exploratory factor analysis: a tutorial on parallel analysis. Organizational Research Methods, 7(2), 191-205.

Hazan, C. \& Shaver, P. (1987) Romantic Love conceptualized as an attachment process. Journal of Personality and Social Psychology, 52(3), 511-524.

Hendin, H., \& Cheek, J. (1997). Assessing hypersensitive narcissism: A reexamination of Murray’s Narcissism Scale. Journal of Research in Personality, 31, 588-599.

Hesse, E. (1996). Discourse, memory and the Adult Attachment Interview: A note with emphasis on the emerging cannot classify category. Infant Mental Health, 17, 4-11.

Hesse, E., \& Main, M. (1999). Second-generation effects of unresolved trauma in nonmal treating parents: Dissociated and frightened and threatening parental behavior. Psychoanalytic Inquiry, 19, 481-540.

Hesse, E., \& Main, M. (2000). Disorganization in infant and adult attachment: Description, correlates, and implications for developmental psychopathology. Journal of the American Psychoanalytic Association, 48, 1097-1127.

Hesse, E., \& Main, M. (2006). Frightened, threatening and dissociative behavior in low-risk samples: Description, discussion and interpretations. Development and Psychopathology, 18, 309-343.

Hibbard, S. (1992). Narcissism, shame, masochism, and object relations: An exploratory correlational study. Psychoanalytic Psychology, 9, 489-508. 
Hinde, R. A. (1974). Biological bases of human social behavior. New York: McGraw-Hill.

Horton, R. S., Bleau, G., \&Drwecki, B. (2006). Parenting narcissus: What are the links between parenting and narcissism? Journal of Personality, 74, 345-376.

Houlcroft, L., Bore, M., \& Munro, D. (in press). Three faces of narcissism. Personality and Individual Differences.

Hrdy, S. B. (2009). Mother and Others: The evolutionary origins of mutual understanding. Cambridge, MA: The Belknap Press of Harvard University.

Hu, L., \& Bentler, P. M. (1999). Cutoff criteria for fit indexes in covariance structure analysis: Conventional criteria versus new alternatives. Structural Equation Modeling, 6, 1-55.

Izard, C. E. (1972). Patterns of emotions: A new analysis of anxiety and depression. New York: Academic Press.

Josephs, L. (1992). The treatment of an adult survivor of incest: A self psychological perspective. American Journal of Psychoanalysis, 52, 201-212.

Kaiser, H. (1974). An index of factorial simplicity. Psychometrika, 39, 31-36.

Kaufman, G. (1989). The Psychology of Shame: Theory and treatment of shame-based syndromes. New York: Springer.

Kline, P. (2000). The handbook of psychological testing (2nd ed.). London: Routledge.

Kline, R.B. (2005). Principles and Practice of Structural Equation Modeling (3rd Ed). Guilford Press: New York, NY.

Kohut, H. (1971). The analysis of the self. Chicago, IL: The University of Chicago Press.

Kohut, H. (1977). The restoration of the self. New York: International University Press. 
Kohut, H. (1980). On empathy: Selected problems in self psychological theory. In P. Ornstein (Ed), The search for the self, vol. 4 (pp. 489-523). Madison, CT: International Universities Press.

Kohut, H. (1984). How does analysis cure? Chicago: University of Chicago Press.

Kohut, H., \& Wolf, E. (1978). The disorders of the self and their treatment: An outline. International Journal of Psychoanalysis, 59,413-425.

Lanyon, R. I., \& Carle, A. C. (2007). Internal and external validity scores on the Balanced Inventory of Desirable Responding and the Paulhus Deception Scales. Educational and Psychological Measurement, 67(5), 859-876.

Lee, R. R. \& Martin, J. C. (1991). Psychotherapy after Kohut. Hillsdale, NJ: The Analytic Press.

Lee, R. M., \& Robbins, S. B. (1995). Measuring belongingness: The social Connectedness and the social assurance scales. Journal of Counseling Psychology, 42, 232-241.

Lee, R. M., \& Robbins, S. B. (1998). The relationship between social connectedness and anxiety, self-esteem, and social identity. Journal of Counseling Psychology, 45, 338-345.

Lee, R. M., \& Robbins, S. B. (2000). Understanding social connectedness in college women and men. Journal of Counseling and Development, 78,484-491.

Lee, R. M., Draper, M., \& Lee, S. (2001). Social connectedness, dysfunctional interpersonal behaviors, and psychological distress: Testing a mediator model. Journal Of Counseling Psychology, 48(3), 310-318. doi:10.1037/0022-0167.48.3.310.

Leone, C. (2001). Toward a more optimal selfobject milieu: Family psychotherapy from the perspective of self psychology. Clinical Social Work Journal, 29, 291-306.

Lessem, P. A. (2005). Self Psychology. Lanham, MD: Rowman \& Littlefield Publishing Group. 
Lewis, H.B. (1980). "Narcissistic personality" or "Shame-prone superego mode". Comparative Psychotherapy, 1, 59-80.

Lichtenberg, J. D. (1988). A theory of motivational-functional systems as psychic structures. Journal of the American Psychoanalytic Association, 36, 57-72.

Lichtenberg, J., Lachmann, F., \& Fosshage, J. (1992). Self and Motivational Systems: Toward a Theory of Psychoanalytic Technique. Hillsdale, NJ: The Analytic Press.

Linehan, M. M. (1993). Cognitive-Behavioral Treatment of Borderline Personality Disorder. New York: Guildford Press.

Liotti, G. (2004). Trauma, dissociation, and disorganized attachment: Three strands of a single braid. Psychotherapy: Theory, Research, Practice, Training, 41, 472-486.

MacCallum, R. C., Widaman, K. F., Zhang, S> B., \& Hong, S. H. (1999). Sample size in factor analysis. Psychological Methods, 4(1), 84-99.

Magai, C, Hunziker, J., Mesias, W., \& Culver, L. C. (2000). Adult attachment styles and emotional biases. International Journal of Behavioral Development, 24, 301 - 309.

Main, M. (1973). Play, exploration and competence as related to child-adult attachment. (Unpublished doctoral dissertation). Baltimore, MD: Johns Hopkins University.

Main, M., \& Goldwyn, R. (1984). Adult attachment scoring and classification system. Version 1. (Unpublished manuscript). University of California, Berkeley.

Main, M. \& Hesse, E. (1990). Parents' unresolved traumatic experiences are related to infant disorganized attachment status: Is frightened and/or frightening parental behavior the linking mechanism? In M. T. Greenberg, D. CIcchetti, \& E. M. Cummings (Eds.), Attachment in preschool years: Theory, research and intervention (pp. 161-182). Chicago, IL: University of Chicago Press. 
Main, M., Kaplan, N., \& Cassidy, J. (1985). Security in infancy, childhood and adulthood: A move to the level of representation. In I. Bretherton \& E. Waters (Eds.), Growing points of attachment theory and research. Monographs of the Society for Research in Child Development, 50 (1-2, Serial No. 209), 66-104.

Main, M. \& Solomon, J. (1986). Discovery of anew, insecure-disorganized/disoriented attachment pattern. In T. B. Brazelton \& M. Yogman (Eds), Affective development in infancy, pp.95-124. Norwood, New Jersey: Ablex.

Marschall, D. E., Sanftner, J., \& Tangney, J. P. (1994). The State Shame and Guilt Scale. In J. P. Tangney \& R. L Dealing (Eds.), Shame and guilt (pp. 239). New York: The Guilford Press.

Mason, W.A. and Mendoza , S. P. (1998). Generic aspects of primate attachments: Parents, offspring and mates. Psychoneuroendocrinology, 24, 765-778

McLean, J. (2007). Psychotherapy with a narcissistic patient using Kohut's self psychology model. Psychiatry (Edgmont), 4(10), 40-7.

Mikulincer, M., \& Florian, V. (1998). The relationship between adult attachment styles and emotional and cognitive reactions to stressful events. In J. A. Simpson \& W. S. Rholes (Eds.), Attachment theory and close relationships (pp. 143-165). New York: Guilford.

Miller, A. (1981). The drama of the gifted child. New York: Basic Books.

Miller, W. R. \&Rollnick, S. (2002). Motivational Interviewing (2nd ed.). New York: The Guilford Press.

Miller, J. D., Dir, A., Gentile, B., Wilson, L., Pryor, L. R., \& Campbell, W. K. (2010). Searching for a vulnerable dark triad: Comparing factor two psychopathy, vulnerable narcissism, and borderline personality disorder. Journal of Personality, 78, 1529-1564. 
Miller. J. D., Hoffman, B. J., Gaughan, E. T., Gentile, B., Maples, J., \& Campbell, W. K. (2011). Grandiose and vulnerable narcissism: A nomological network analysis. Journal of Personality, 79(5), 1013-1042.

Mollon, P. (2003). Foreword. In M. Cortina \& M. Marrone (Ed), Attachment Theory and the Psychoanalytic Process. Mondon: Whurr, pp. xvii-xix.

Morey, L., Waugh, M., \& Blashfield, R. (1985). MMPI scales for the DSM-III personality disorders: Their derivation and correlates. Journal of Personality Assessment, 49, 245251.

Morrison, A. (1994). The breadth and boundaries of a self-psychological immersion in shame: A one-and-a-half person perspective. Psychoanalytic Dialogues, 4(1), 19-36.

Morrison, A. (1986). Shame, ideal self, and narcissism. In A. P. Morrison (Ed.), Essential Papers on Narcissism. New York: New York University Press.

Mundfrom, D. J., Shaw, D. G., \& Tian, L. K. (2005). Minimum sample size recommendations for conducting factor analysis. International Journal of Testing, 5, 159-168.

Munro, D., Bore, M., \& Powis, D. (2005). Personality factors in professional ethical behavior: Studies of empathy and narcissism. Australian Journal of Psychology, 57(1), 49-60.

Muslin, H. (1993). Psychotherapy of the Elderly Self. London: Routledge.

Nathanson, D. L. (1992). Shame and pride: Affect, sex and the birth of the self. New York: Norton.

O’Brien, M. L. (1987). Examining the dimensionality of pathological narcissism: Factor analysis and construct validity of the O'Brien Multiphasic Narcissism Inventory. Psychological Reports, 62, 879-882. 
O'Brien, M. L. (1988). Further evidence of the validity of the O'Brien Multiphasic Narcissism Inventory. Psychological Reports, 62, 878-882.

Ogawa, J. R., Sroufe, L. A., Weinfeild, N. S., Carlson, E.A., \& Egeland, B. (1997). Development and the fragmented self: Longitudinal study of dissociative symptomatology in a nonclinical. Development and Psychopathology, 9, 855-879.

Otway, L. J., \& Vignoles, V. L. (2006). Narcissism and childhood recollections: A quantitative test of psychoanalytic predictions. Personality and Social Psychology Bulletin, 32, 104116.

Palombo, J. (1983). Borderline conditions: A perspective from self psychology. Clinical Social Work Journal, 11, 323-338.

Paradis, B. (1993). A self psychological approach to the treatment of gay men with AIDS. Clinical Social Work Journal, 21, 405-416.

Paulhus, D. L. (1998). Paulhus deception scales (PDS): The balanced inventory of desirable responding-7: User's manual. North Tonawanda, N.Y.: Multi-Health Systems.

Paulhus, D. L., \& Williams, K. M. (2002). The dark triad of personality: Narcissism, Machiavellianism, and psychopathy. Journal of Research in Personality, 36, 556-563.

Pepper, J., \& Strong, P. (1985). Judgmental subscales for the MF scale of the MMPI. Unpublished manuscript.

Pfenig, D. W., \& P. W. Scherman. (1995). Kin recognition. Sci. Am. (June 17) :98-103.

Pincus, A. L., Ansell, E. B., Pimentel, C. A., Cain, N. M., Wright, A., \& Levy, K. (2009). Initial construction and validation of the Pathological Narcissism Inventory. Psychological Assessment, 21, 365-379. 
Polan, H. J., \& Hofer, M. A., (2008). Psychobiological origins of infant attachment and its role in development. In J. Cassidy \& P. R. Shaver (Eds.), Handbook of attachment: Theory, research, and clinical applications (2nd ed., pp. 158-172). New York: The Guilford Press.

Prooijen, J. V., \& Kloot, V. D. (2001). Confirmatory analysis of exploratively obtained factor structures. Educational and Psychological Measurement, 61(5), 777-792.

Pulver, S. (1986). Narcissism: The term and the concept. In A. P. Morrison (Ed.), Essential Papers on Narcissism. New York: New York University Press

Raskin, R., \& Hall, C. (1979). A narcissistic personality inventory. Psychological Reports, 45, 590.

Raskin, R., \& Terry, H. (1988). A principle-component analysis of the narcissistic personality inventory and further evidence of its construct validity. Journal of Personality and Social Psychology, 54, 890-902.

Raskin, R., \& Novacek, J. (1989). An MMPI description of the narcissistic personality. Journal of Personality Assessment, 53, 66-80.

Rathvon, N., \& Holmstrom, W. (1996). An MMPI-2 portrait of narcissism. Journal of Personality Assessment, 66, 1-19.

Raykov, T. \& Marcoulides, G.A. (2006). A first course in structural equation modeling ( ${ }^{\text {nd }} E d$.). Lawrence Erlbaum: Mahwah, NJ.

Reich, A. (1986). Pathologic forms of self-esteem regulation. In A. P. Morrison (Ed.), Essential Papers on Narcissism (pp. 44-60). New York: New York University Press. 
Robbins, S. B., \& Patton, M. J. (1985).Self psychology and career development: Construction of the superiority and goal instability scales. Journal of Counseling Psychology, 32(2), 221231.

Robbins, S. B. (1987). Predicting change in career indecision from a self-psychology perspective. The Career Development Quarterly, 35(4), 289-296.

Robbins, S. B., \& Schwitzer, A. M. (1988). Validity of the Superiority and Goal Instability Scales as predictors of women's adjustment to college life. Measurement and Evaluation in Counseling and Development, 21, 117-123.

Robbins, S. B. (1989). Validity of the Superiority and Goal Instability Scales as measures of defects in the self. Journal of Personality Assessment, 53, 122-132.

Robbins, S. B., Lesse, K. P., \& Herrick, S. M. (1993). Interactions between goal instability and social support on college freshmen adjustment. Journal of Counseling and Development, 71, 343-348.

Robbins, S. B., Payne, E. C., \& Chartrand, J. M. (1990). Goal instability and later life adjustment. Psychology and Aging, 5(3), 447-450.

Robertson, J., \& Blowlby, J. (1952). Responses of young children to separation from their mothers. Courrier of the International Children's Centre, Paris, II, 131-140.

Ronningstam, E., \& Gunderson, J. (1990). Identifying narcissistic personality disorder. American Journal of Psychiatry, 147, 918-922.

Rose, P. (2002). The happy and unhappy faces of narcissism. Personality and Individual Differences, 33, 379-392. 
Rutter, M., Kreppner, J., \& Sonuga-Barke, E. (2009). Emmanuel Miller Lecture: Attachment insecurity, disinhibited attachment, and attachment disorders: Where do research findings leave the concepts? Journal of Child Psychology \& Psychiatry, 50(5), 529-543.

Sartre, J. P. (2007). Nausea. (L. Alexander, Trans.). New York, NY: New Directions Books. (Original work published 1938).

Sawrie, S. M., Watson, P. J., Sherbak, J. F., Greene, R. L., \& Arredondo, R. (1997). Alcoholism and narcissism: Assessing a presumed relationship with the MMPI-2. Alcoholism Treatment Quarterly, 15(3), 1-14.

Scott, K. J., \& Robbins, S. B. (1985). Goal instability: Implications for academic performance among students in learning skills courses. Journal of College Student Personnel, 26(2), 129-133.

Sedikides, C., Rudich, E. A., Gregg, A. P., Kumashiro, M., \&Rusbult, C. (2004). Are normal narcissists psychologically healthy? Self-esteem matters. Journal of Personality and Social Psychology, 87, 400-416.

Seidler, G. H. (1997). From object-relations theory to the theory of alterity. American Journal of Psychotherapy, 51(3), 343-356.

Serkownek, K. (1975). Subscales for the scales of 5 and 0 of the MMPI. Unpublished manuscript.

Siegel, A. M. (1996). Heinz Kohut and the psychology of the self. London: Psychology Press.

Silverstein, M. L. (2007). Disorders of the Self. Washington, DC: American Psychological Association.

Silverstein, M. L. (1999). Self psychology and diagnostic assessment. London: Routledge. 
Simpson, J. A., \& Rholes, W. S. (1998). Attachment theory and close relationships. New York: Guilford Press.

Smolewska, K., \& Dion, K. L. (2005). Narcissism and adult attachment: A multivariate approach. Self and Identity, 4(1), 59-68.

Steiger, J. H. (1980). Tests for comparing elements of a correlation matrix. Psychological Bulletin, 87, 245-251.

Stern, D. N. (2006). . Some implications of infant research for psychoanalysis. In A. M. Cooper (Ed), Contemporary Psychoanalysis in America. Washington, DC: American Psychiatric Publishing, pp. 637-641.

Stone, W. (1992). The place of self psychology in group psychotherapy: A status report. International Journal of Group Psychotherapy, 42, 335-350.

Stolorow, R. D., Brandchaft, B., \& Atwood, G. E. (1983). Intersubjectivity in psychoanalytic treatment, with special reference to archaic states. Bulletin of the Meninger Clinic, 47, 117-128.

Summers, F. (1994). Object relation theories and psychopathology. Hillsdale, NJ: Analytic Press.

Suth, R. (1996). A Self-psychological approach to child therapy: A case study. In A. Goldberg, (ed.), Progress in Self Psychology, Volume 12. Hillsdale, NJ: Analytic Press, 183-199.

Tabachnick, B. G., \& Fidell, L. S. (2001). Using Multivariate Statistics. Boston: Allyn and Bacon.

Tangney, J. P., \& Dealing, R. L. (2002). Shame and guilt. New York: The Guilford Press. Tangney, J. P. (1995). Recent advances in the empirical study of shame and guilt. American Behavioral Scientist, 38, 1132-1145. 
Taylor, C.M. (1995). The profile of narcissistic dispositions (POND). Unpublished Doctoral Dissertation, University of British Columbia.

Teicholz, J. (1999). Kohut, Loewald, and the postmoderns: A comparative study of the self and relationship. Hillsdale, NJ: Analytic Press.

Toplin, M. (2002). Doing psychoanalysis of normal development: Forward edge transferences. In A Goldberg (Ed.), Progress in self psychology: Vol. 18. Postmodern self psychology (pp. 167-190). Hillsdale, NJ: Analytic Press.

Trop, J., \& Stolorow, R. (1992). Defense analysis in self psychology: A developmental view. Psychoanalytic Dialogues, 2(4), 427-442.

Ulman, R., \& Paul, H. (1989). A self-psychological theory and approach to treating substance abuse disorders: The "intersubjective absorption" hypothesis. In A. Goldberg (ed.), Dimensions of Self Experience: Progress in Self Psychology, Volume 5. Hillsdale, NJ: Analytic Press, 121-141.

Velicer, W. F., \& Jackson, D. N. (1990). Component Analysis Versus Common Factor-AnalysisSome Further Observations. Multivariate Behavioral Research, 25(1), 97-114.

Weinfeld, N. S., Sroufe, L. A., Egeland, B., Carlson, E. A. (2008). Individual differences in infant-caregiver attachment: Conceptual and empirical aspects of security. In J. Cassidy \& P. R. Shaver (Eds.), Handbook of attachment: Theory, research and clinical applications (2nd ed., pp. 78-101). New York: The Guilford Press.

Westen, D., Lohr, N., Silk, K., Kerber, K., \& Goodrich, S. (1985). Measuring object relations and social cognition using the TAT: Scoring Manual. Unpublished manuscript, University of Michigan. 
Widiger, T. A., Mangine, S., Corbitt, E. M., Ellis, C. G., \& Thomas, G. V. (1995). Personality Disorder Interview-IV: A semi-structured interview for the assessment of personality disorders. Odessa, Fl: Psychological Assessment Resources, Inc.

Williams, K. L., \& Galliher, R. V. (2006). Predicting depression and self-esteem from social connectedness, support, and competence. Journal of Social and Clinical Psychology, 25(8), 855-874.

Wink, P., \& Gough, H. G. (1990). New narcissism scales for the California Personality Inventory and MMPI. Journal of Personality Assessment, 54, 446-462.

Wink, P. (1991). Two faces of narcissism. Journal of Personality and Social Psychology, 61, 590-597.

Wink, P. (1992). Three narcissism scales for the California Q-set. Journal Of Personality Assessment, 58(1), 51-66.

Wink, P., \& Donahue, K. (1996).Implications of college-age narcissism for psychosocial functioning at midlife: Findings from a longitudinal study of women. Journal of Adult Development, 2, 73-85.

Wolf, E. (1988). Treating the Self: Elements of clinical self psychology. New York: Guildford Press.

Youngstrom, E. A., \& Green, K.W. (2003). Reliability generalization of self-report of emotions when using the differential emotions scale. Educational and Psychological Measurement, 63(2), 279-295. 


\section{ABSTRACT \\ AN ANALYSIS OF SELF: \\ THE DEVELOPMENT AND ASSESSMENT OF A MEASURE OF SELFOBJECT \\ NEEDS}

by

\section{EAMONN ARBLE}

August 2014

Advisor: Dr. Douglas Barnett

Major: Psychology

Degree: Doctor of Philosophy

The theory of self psychology as advanced by Heinz Kohut (1971, 1977, 1984) emphasized the importance of early developmental experiences, most centrally, the empathic attunement of caregivers as key nutrients for fostering the capacity for healthy self-regulation and interpersonal relations. Kohut elaborated three critical selfobject needs (i.e., idealizing, mirroring, and twinship) that were ideally satiated by these early experiences, deeming the fulfillment of these needs as essential for developing a healthy sense of self, capable of sustaining ambitions, self-soothing, and caring for others and oneself. In the case of psychopathology, these needs were theorized to have gone unmet, leaving the individual with deficits in their ability to regulate self-esteem, and thereby rendering them vulnerable to mood and behavioral dysregulation, as well as difficulty sustaining satisfying intimate relations, especially in the face of life's stressors. Although Kohut's theory has been embraced as quite useful by many mental health professionals, particularly those with a psychodynamic affiliation, there has been relatively scant empirical research examining its underlying hypotheses. It is the present author's assertion that a primary reason for the scarcity of empirical attention devoted to 
Kohut's theory has been due to challenges operationalizing his concepts, which has led to a lack of adequate measures of his key constructs, especially his idea of the selfobject needs. To address these shortcomings, the present research sought to develop a self-report measure, named the AESOP, capable of assessing the selfobject needs identified by Kohut. The proposed strategy was designed to yield a measure that could reliably and validly assess individual differences in the selfobject needs of idealizing, mirroring, and twinship. Experts in the field of self psychology were recruited to assist in the development of the present measure. Across two studies involving 738 and 712 respondents, participants completed an online survey hosted by the university's secure research portal. The first study involved a survey including only the present measure, while the second study's survey consisted of the present measure and other measures of interest. Exploratory and confirmatory factor analyses offered strong evidence in support of the proposed three-factor structure of the measure. Cluster analysis and examination of the correlational results offered additional support for the present measure's validity, both in regards to discriminant and convergent validity. The theoretical implications of these findings are explored. 


\section{AUTOBIOGRAPHICAL STATEMENT}

\section{EDUCATION}

\begin{tabular}{cccc}
\hline INSTITUTION AND LOCATION & DEGREE & YEAR & FIELD OF STUDY \\
\hline $\begin{array}{c}\text { University of Michigan, } \\
\text { Dearborn, MI }\end{array}$ & B.A. & 2004 & $\begin{array}{c}\text { Philosophy, } \\
\text { Psychology }\end{array}$ \\
\hline $\begin{array}{c}\text { Eastern Michigan University, } \\
\text { Ypsilanti, MI }\end{array}$ & M.S. & 2009 & $\begin{array}{c}\text { Psychology, } \\
\text { General Clinical Track }\end{array}$ \\
\hline $\begin{array}{c}\text { Wayne State University, } \\
\text { Detroit, MI }\end{array}$ & Ph.D. & Current & Clinical Psychology \\
\hline
\end{tabular}

\section{CLINICAL PLACEMENTS}

1. Internship - John D. Dingell VA Medical Center: Detroit, MI

2. Wayne State Psychology Clinic (Clinical Training Assistant): Detroit, MI

3. Life Stress Center, Detroit Receiving Hospital: Detroit, MI

4. Center for Forensic Psychiatry: Ann Arbor, MI

5. University of Michigan-Dearborn Student Counseling Center: Dearborn, MI

\section{SELECTED PUBLICATIONS}

Arble, E., Kuentzel, J., \& Barnett, D. (2014). Convergent validity of the Integrated Visual and Auditory Continuous Performance Test (IVA+Plus): Associations with working memory, processing speed, and behavioral ratings. Archives of Clinical Neuropsychology.

Barnett, D., Heinze, H., \& Arble, E. (2013). Risk, resilience, and the Rorschach: A longitudinal study of children who experienced sexual abuse. Journal of Personality Assessment.

Arnetz, B.B., Arble, E., Backman, L., Lynch, A., \& Lublin, A. (2013). Assessment of a prevention program for work-related stress among urban police officers. Int Arch Occup Environ Health, 86(1), 79-88.

Kuentzel, J.G., Arble, E., Boutros, N., Chugani, D., \& Barnett, D. (2012). Non-suicidal self injury in an ethnically diverse college sample. American Journal of Orthopsychiatry, 82(3), 291-297.

Arble, E., Dean, C., Tolchinsky, A., \& Huprich, S. K. (2008). In S. K. Huprich (Ed.), Theoretical and Treatment Principles for Narcissistic Personalities. Lanham, MD: Jason Argosy.

Gauger, P. G., Hauge, L. S., Andreatta, P. B., Hamstra, S. J., Hillard, M. L., Arble, E. P., Kasten, S. J., Mullan, P. B., Cederna, P. S., \& Minter, R. M. (2010). Laparoscopic Simulation Training with Proficiency Targets and Progress Reports Improves Practice and Performance of Novice Surgeons. American Journal of Surgery, 199(1), 72-80. 